\title{
WestVirginiaUniversity
}

THE RESEARCH REPOSITORY @ WVU

Graduate Theses, Dissertations, and Problem Reports

2010

\section{Nanofabrication of Optofluidic Photonic Crystal Resonators for Biosensing}

Henry Bolivar Andagana Junta

West Virginia University

Follow this and additional works at: https://researchrepository.wvu.edu/etd

\section{Recommended Citation}

Andagana Junta, Henry Bolivar, "Nanofabrication of Optofluidic Photonic Crystal Resonators for Biosensing" (2010). Graduate Theses, Dissertations, and Problem Reports. 3052.

https://researchrepository.wvu.edu/etd/3052

This Thesis is protected by copyright and/or related rights. It has been brought to you by the The Research Repository @ WVU with permission from the rights-holder(s). You are free to use this Thesis in any way that is permitted by the copyright and related rights legislation that applies to your use. For other uses you must obtain permission from the rights-holder(s) directly, unless additional rights are indicated by a Creative Commons license in the record and/ or on the work itself. This Thesis has been accepted for inclusion in WVU Graduate Theses, Dissertations, and Problem Reports collection by an authorized administrator of The Research Repository @ WVU. For more information, please contact researchrepository@mail.wvu.edu. 


\title{
Nanofabrication of Optofluidic Photonic Crystal Resonators for Biosensing
}

\author{
by \\ Henry Bolivar Andagana Junta \\ Thesis submitted to the \\ College of Engineering and Mineral Resources at \\ West Virginia University \\ in partial fulfillment of the requirements \\ for the degree of \\ Master of Science \\ in
}

Electrical Engineering

Committee members:

Prof. Xian-An Cao

Prof. Jeremy Dawson

Prof. Larry Hornak

Lane Department of Computer Science and Electrical Engineering

Morgantown, West Virginia, 26506

2010

Keywords: Nanofabrication, Electron Beam Lithography, Inductively Coupled Plasma Etching, Glancing angle deposition, Photonic Crystal, Biosensor 


\begin{abstract}
Nanofabrication of Optofluidic Photonic Crystal Resonators for Biosensing
\end{abstract}

Henry Andagana

Advances in nanofabrication have made possible the development of novel nanophotonics based on Photonic Crystal (PhC) structures. Different types of biosensors have been proposed to take advantage of the unique optical properties of PhCs, which, when combined with microfluidics, provide an enabling approach for biomolecule analysis. In this thesis, nanofabrication of a new biosensor structure integrating a 2D PhC nanocavity resonator with an optofluidic channel is described. The recipes for e-beam lithography of $\mathrm{PhC}$ and $\mathrm{PhC}$ nanocavity patterns were optimized by carefully tuning the e-beam dose as well as control of the resist baking and development conditions. Two PhC fabrication processes based on pattern transfers by lift-off and etch-back were compared. The plasma etching of $\mathrm{Si}, \mathrm{GaN}$, and ITO were optimized to obtain fast etching rates, vertical profiles as well as high etch selectivities over different hard masks including $\mathrm{Si}_{3} \mathrm{~N}_{4}, \mathrm{SiO}_{2}$, and $\mathrm{Ni}$. Si, GaN and ITO PhC structures with airhole diameters in the range of 100-200 nm were fabricated using the developed processes. To facilitate the integration of the $\mathrm{PhC}$ structures with microfluidic channels, the $\mathrm{PhC}$ airholes were sealed with a $\mathrm{SiO}_{2}$ thin film formed by glancing angle deposition. By gradually reducing the flux angle toward normal during deposition, we successfully deposited uniform $\mathrm{SiO}_{2}$ capping layers with minimal material extended down into the $\mathrm{PhC}$ airholes. Finally, suspended $\mathrm{Si} \mathrm{PhC}$ slabs were fabricated on a Si-on-Oxide substrate using an integrated procedure which consists of e-beam lithography, pattern transfer into $\mathrm{Si}$ by $\mathrm{CF}_{4}$ plasma etching, and a selective etch of the underlying $\mathrm{SiO}_{2}$ sacrificial layer in HF. The suspended $\mathrm{PhC}$ structure provides an important test bed for measuring resonant fluorescence of biomolecules in $\mathrm{PhC}$ nanocavities in the IR range. 


\section{ACKNOWLEDGEMENTS}

It is my great pleasure that I thank the many people who helped to make this work possible.

First and foremost, I want to extend my thanks and gratitude to my family for all the love and support that they have given to me throughout my life. Without their support and encouragement, I would have never made it this far. Todos mis mas elevados agradecimientos para el sr Oso que dia a dia me brinda su apoyo y consejo para seguir adelante en mi vida cotidiana y profesional. Igual a mis hermanitas pequenias que nunca me dieron su espalda sino todo su apoyo y carinio durante el desarrollo de mi carrera.

I would like to thank my advisor and the chairperson of my committee, Dr. Xian-An Cao, for his continuous support and trust throughout my graduate studies at West Virginia University. I am very grateful for the many informative discussions, the knowledge that he shared with me, and his ultimate research experience that motivated me to continue exploring the amazing world of micro- and nanofabrication.

I would also like to thank Dr. Jeremy Dawson. Dr. Dawson has been a constant source of motivation and support throughout my research work. His knowledge and rich experience in the fields of Photonics and Optics have helped me to develop a solid understanding that was essential for my research work.

I would also like to thank Dr. Larry Hornak for his advice and needed guidance for my research work. I am also very grateful to Dr. Kolin Brown. He provided me with the necessary training sessions and assistance with the equipments available in the WVNano Shared Cleanroom Facility.

Many thanks to Dr Felio Perez, Dr. Sergei Urazhdin, Mr Erick Shires, and Ms. Adrienne McGraw for all the unconditional help when learning how to operate the equipments located in the Physic and the Electrical Engineering Departments.

In addition, I want to extend my heartfelt thanks to the students that worked in the cleanroon along with me and shared their expertise and their tireless enthusiasm. They are Yi Yang, Sridhar Kuchibhatla, Bashar Hamza, and Hyma Yalamanchili.

Finally and most importantly, I would like to thank God for all He has given me. Without his blessings, none of this would have been possible. 


\section{TABLE OF CONTENTS}

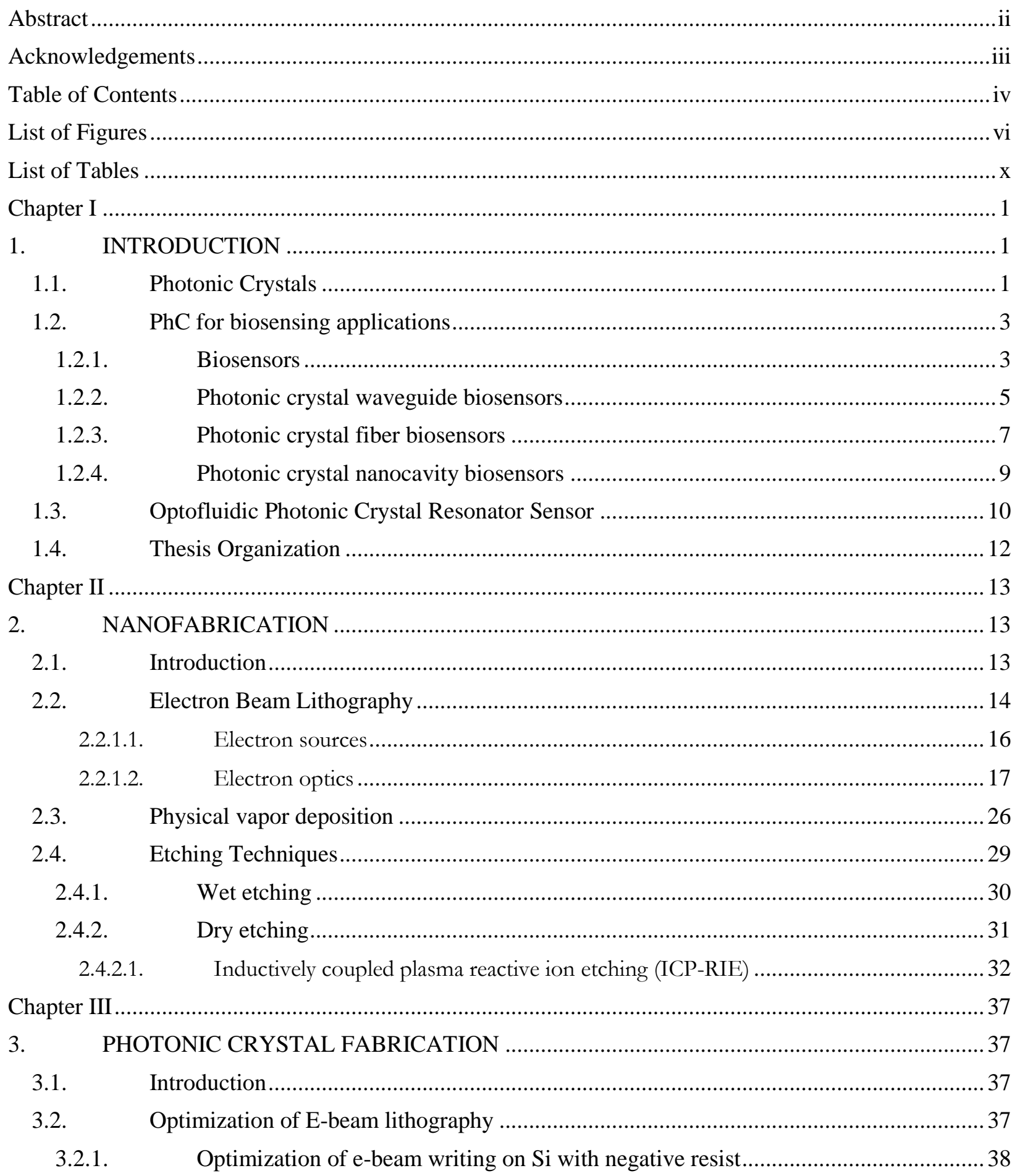


3.2.2. Optimization of e-beam writing on $\mathrm{GaN}$ with positive resists............................... 40

3.2.3. Optimization of e-beam writing on $\mathrm{GaN}$ with negative resists .................................... 40

3.3. Comparison of Lift-off and Etch-back processes for hard mask patterning ...................... 44

3.3.1. Hard mask patterning by Lift-off .................................................................... 44

3.3.2. Hard mask patterning by Etch-back ....................................................................... 46

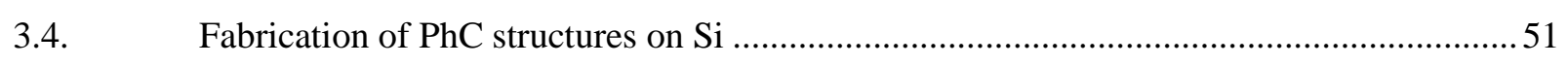

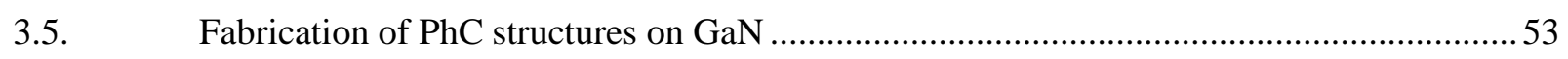

3.5.1. Fabrication of $\mathrm{PhCs}$ on $\mathrm{GaN}$ using hard masks formed by lift-off .............................54

3.5.2. Fabrication of $\mathrm{PhC}$ on $\mathrm{GaN}$ using hard masks formed by two-step pattern transfer...57

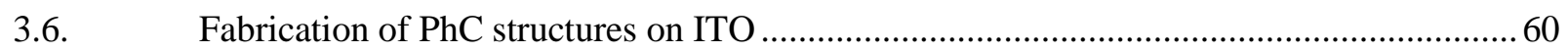

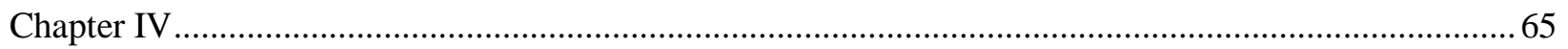

4. SEALING OF PhC AIRHOLES BY GLANZING ANGLE DEPOSITION ...........................65

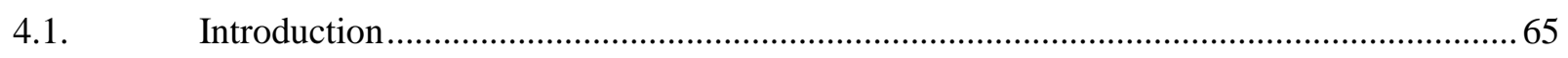

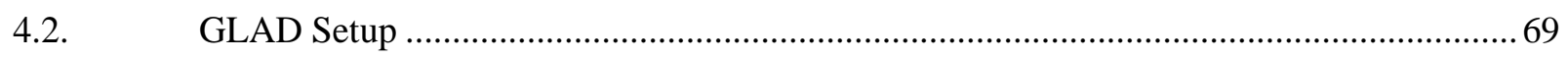

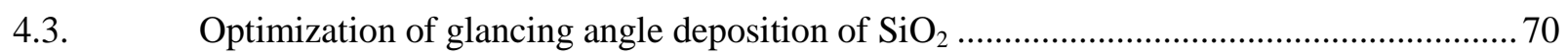

4.4. $\quad \mathrm{PhC}$ sealing by GLAD with substrate rotation............................................................. 72

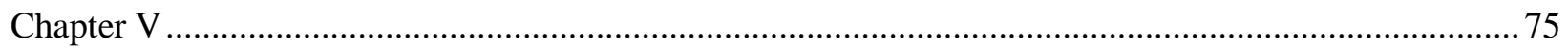

5. FABRICATION OF SUSPENDED PhC NANOCAVITY SLAB FOR BIOSENSING

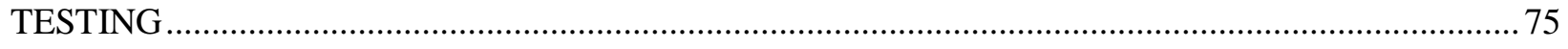

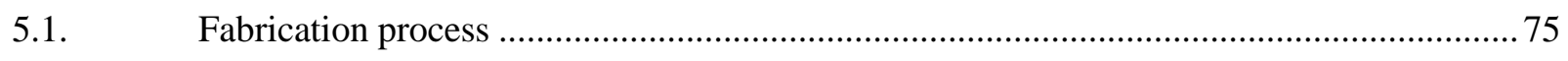

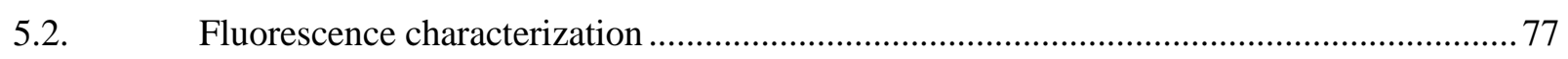

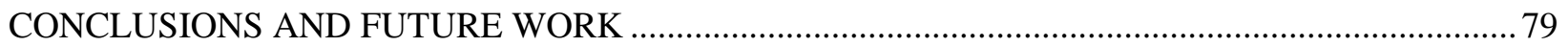

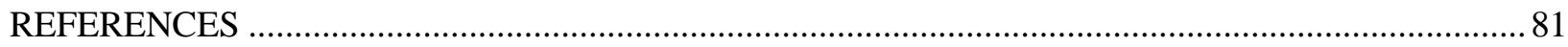

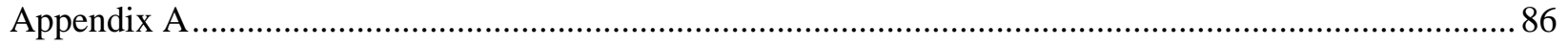

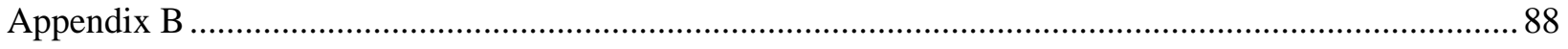




\section{LIST OF FIGURES}

Figure 1-1: (a) a 2D Photonic Crystal slab structure, (b) dimensions to be considered in a PhC structure, (c) dispersion diagram of a hexagonal lattice $\mathrm{PhC}^{4}$, (d) a point defect introduced in the center of a $\mathrm{PhC}$

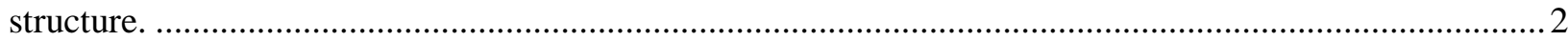

Figure 1-2: Multiple expertise put together to achieve successful biosensors. ${ }^{11}$................................... 3 Figure 1-3: Biosensor: (a) surface modified-transducer, reactive to specific analyte, (b) principle of operation of a biosensor in general. ${ }^{11}$

Figure 1-4: (a) $\mathrm{PhC}$ waveguide slab on SOI; missing row of holes constitue the waveguide, (b) transmission spectrum showing shift $\mathrm{s}$ of the cutoff wavelength on the structure, (c) setup for the whole biosensing technique. ${ }^{18}$

Figure 1-5: (a) cross section of PhC fiber with a defect in the center, (b) biofilms attached to the inside of each hole in the fiber, (c) The biomolecular structure attachment of poly-L-lysine (black and red) immobilized onto the silica surface $(\mathrm{SiO} 2), \mathrm{DNA}$ (green) is immobilized on the poly-L-lysine. ${ }^{19}$

Figure 1-6: (a) transmission spectrum for PBS (phosphate buffered saline solution), PLL (poly-L-lysine)

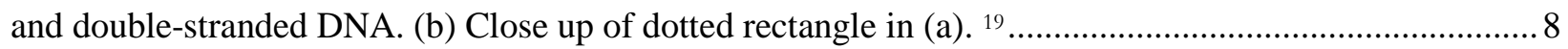
Figure 1-7: (a) top view of the $\mathrm{PhC}$ with the defect and its ridge waveguides at the ends, (b) transmission spectrum for such structure, (c) schematic of the biosensing setup. ${ }^{20}$ 10

Figure 1-8: (a) Top view of an optofluidic $\mathrm{PhC}$ resonator biosensor; (b) cross section schematic of the biosensor; (c) light enhancement is produced by the cavity due to the presence of labeled biomolecules; (d) no light enhancement is produced by unlabeled biomolecule even when they are in the cavity. ......... 11

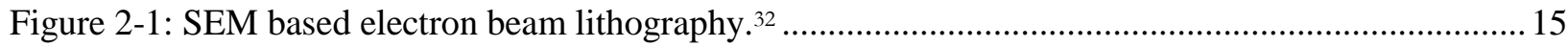

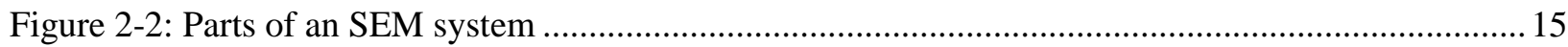

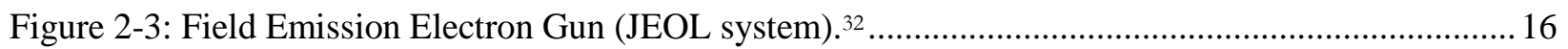

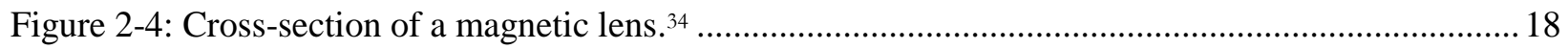

Figure 2-5: Aberrations, diffraction and thermal transverse velocity effects. ${ }^{34}$..................................... 20

Figure 2-6: Quadrupole stigmators, beam cross-section. ${ }^{34}$................................................................ 21

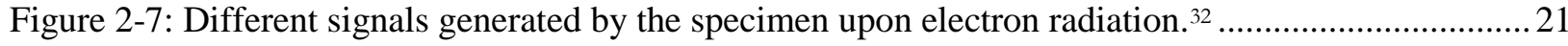


Figure 2-8: Electron scattering: (a) Forward scattering of primary electrons; (b) Forward scattering of secondary electrons; ${ }^{37}$ (c) and (d) Forward and backscattering of electrons in resist and substrate as

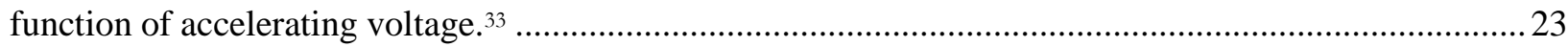

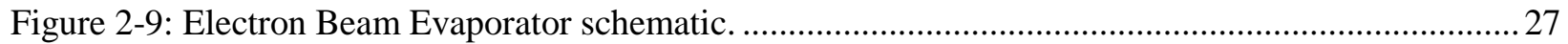

Figure 2-10: Shadow effect on a step in a sample (a). Neither rotated nor heated (b). Rotated and heated. ${ }^{43}$ .28

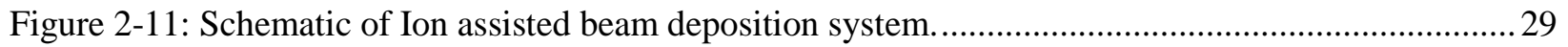

Figure 2-12: Types of etching mechanisms depending on environment pressure. ${ }^{43}$.................................2 29

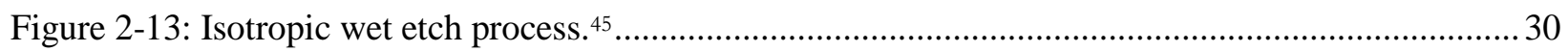

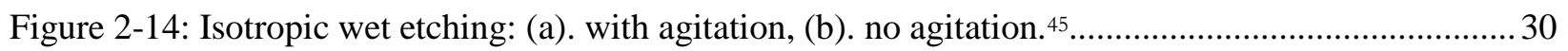

Figure 2-15: Cross section of anisotropic wet etching in: (a) (100) orientation; (b) (110) orientation. ${ }^{45}$... 31

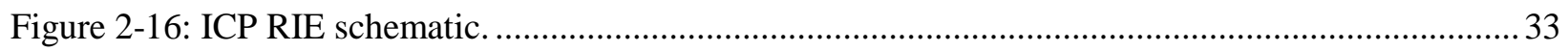

Figure 2-17: (a) Etching process and passivation sidewalls; (b) Scheme of RIE process. ${ }^{45} \ldots \ldots \ldots \ldots \ldots \ldots \ldots \ldots . . . . . . . .34$

Figure 2-18: (a) Etching effect due to non optimized macroparameter; ${ }^{48}$ (b) Loading effect. ${ }^{45}$................. 36

Figure 3-1: Top-view SEM micrograph of the dose-test pattern written and developed on Si................. 39

Figure 3-2: Top-view (left) and $45^{\circ}$-view (right) SEM micrographs of dose characterization with EBL for ma-N 2403: (a) and (b) underexposed patterns; (c) and (d) optimal exposure; (e) and (f) overexposed

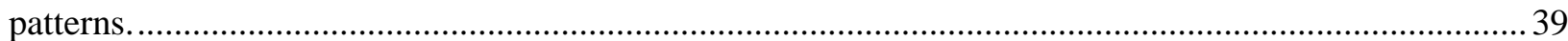

Figure 3-3: Top-view SEM micrographs of dose characterization with EBL for PMMA 950A: (a) underexposed pattern; (b) overexposed pattern; (c) and (d) optimal exposure $370 \mu \mathrm{C} / \mathrm{cm}^{2} \ldots \ldots \ldots \ldots \ldots \ldots . . . . . . .11$

Figure 3-4: Top-view SEM micrographs of PhC pattern on $\mathrm{GaN}\left(65 \mu \mathrm{C} / \mathrm{cm}^{2}\right)$..................................... 41

Figure 3-5: Top-view SEM micrographs of pattern resolution as a function of the lattice constant (a), (d)

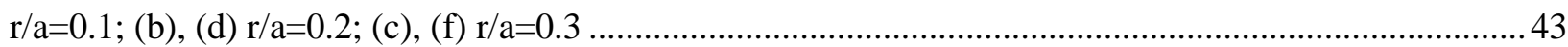

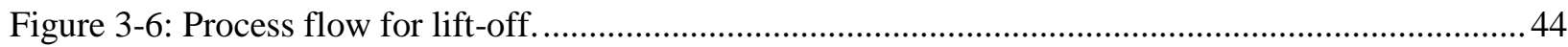

Figure 3-7: Lift-off technique, (a), (b), (c) Top-view SEM micrograph of the patterns written on the negative resist by EBL (d) Top-view SEM micrograph of the Ni metal mask after lift-off and ICP etching, (e) Top-view SEM micrograph of the whole PhC structure on $\mathrm{Si}$; (f) $45^{\circ}$-view SEM micrograph of the final $\mathrm{PhC}$ structures (parameters of fabrication non optimized). ........................................................... 46

Figure 3-8: Etching rates of $\mathrm{SiO}_{2}$ and $\mathrm{Si}_{4} \mathrm{~N}_{3}$ grown by PECVD and Evaporation...................................4 47

Figure 3-9: Process flow for the fabrication of $\mathrm{PhC}$ on $\mathrm{GaN}$ (etch-back approach) ................................ 49

Figure 3-10: SEM micrographs of the PhC fabrication on GaN (etch-back): (a) PhC on PMMA; (b) cured PMMA; (c) ICP etching of Ni; (d) cross section of $\mathrm{PhC}$ on Ni; (e) ICP etching of $\mathrm{SiO}_{2}$; (f) ICP etching of $\mathrm{GaN}$; (g) cross section of $\mathrm{PhC}$ on $\mathrm{GaN}-\mathrm{SiO}_{2}$ mask still on top of the substrate; (h) $\mathrm{SiO}_{2}$ mask removal

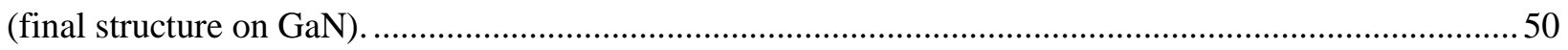


Figure 3-11: Top-view SEM micrographs of PMMA reflow at $140{ }^{\circ} \mathrm{C}$ : (a) edge of the $\mathrm{PhC}$; (b) center of

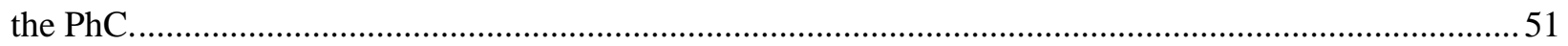

Figure 3-12: Process flow for the fabrication of $\mathrm{PhC}$ on $\mathrm{Si}$....................................................................52

Figure 3-13: SEM micrographs of the fabrication of the $\mathrm{PhC}$ on $\mathrm{Si}$ : (a) $45^{\circ}$ view of $\mathrm{PhC}$ on $\mathrm{Si}$; (b) $45^{\circ}$ view of nanocavity in the PhC structure; (c) cross section of PhC-sidewall profiles; (d) $45^{\circ}$ view of the

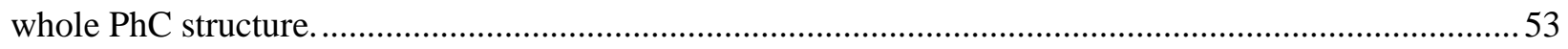

Figure 3-14: Process flow for the fabrication of $\mathrm{PhC}$ on $\mathrm{GaN}$ (lift-off approach) ....................................54

Figure 3-15: SEM micrographs of the $\mathrm{PhC}$ structure on $\mathrm{GaN}$, lift-off process (a) top view of $\mathrm{PhC}$ on the resist layer; (d) top view of Ni metal mask after lift-off; (b) top view of PhC pattern transfer from Ni mask into $\mathrm{GaN}$; (e) top view of final $\mathrm{PhC}$ structure on $\mathrm{GaN}$; (c), (f) final $\mathrm{PhC}$ structures on $\mathrm{GaN}, 45^{\circ}$ view.....56 Figure 3-16: Cross-section SEM micrographs of Etching profiles of GaN structures: (a) and (b) Ni mask too thin for ICP etching process; (c) and (d) PhC etched with Ar based plasma; (e) and (f) PhC etched

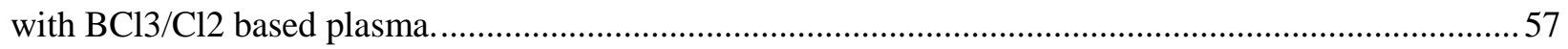

Figure 3-17: Process flow for the fabrication of PhC on GaN (lift-off and bylayer hard mask). ..............58

Figure 3-18: SEM micrographs of the steps for the PhC fabrication on GaN (lift off, experiment B): (a) $\mathrm{PhC}$ on resist ma-N2403; (b) Ni mask after lift-off process; (c) ICP etching of $\mathrm{SiO}_{2}$; (d) ICP etching of $\mathrm{GaN}$; (f) and (g) $45^{\circ}$ view of $\mathrm{PhC}$ on Gan after mask removal

Figure 3-19: Etching rates of ITO based on $\mathrm{BCl}_{3} / \mathrm{Cl}_{2}$ plasma: (a) variation of percentage of $\mathrm{Cl} 2$ in a mix of $\mathrm{BCl} 3 / \mathrm{Cl} 2$; (b) variation of RIE power; (c) variation of ICP power; (d) variation of chamber pressure. .... 61 Figure 3-20: Etching rate of ITO when adding small percentages of $\mathrm{CF}_{4}$ to the $\mathrm{BCL}_{3} / \mathrm{Cl}_{2}$ mix..............62

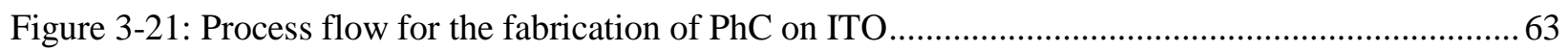
Figure 3-22: SEM micrographs of the fabrication of PhC structures on ITO film: (a) top view of PhC on resist ma-N 2403(75 $\left.\mu \mathrm{C} / \mathrm{cm}^{2}\right)$; (b) 450 view of $\mathrm{PhC}$ on ITO; (c) and (d) cross section of PhC structure on ITO. 64

Figure 4-1: (a) Normal Deposition, (b) Oblique angle deposition, (c) Variables for Oblique deposition. .65 Figure 4-2: (a) deposition of film at right angle $\alpha$ and no substrate rotation (b) deposition of film at $\alpha=$ $75^{\circ}$ and no substrate rotation (tilted nanorod growing) (c) deposition of film at $\alpha=75^{\circ}$ and low substrate rotation (helical growing) (d) deposition of film at $\alpha=75^{\circ}$ and substrate rotation (vertical nanorod growing) (e) deposition of film at $\alpha=75^{\circ}$ and high substrate rotation (irregular surface morphology). ${ }^{62} .67$ Figure 4-3: (a) GLAD schematic diagram; (b) material flux arriving onto the PhC structure by GLAD plus substrate rotation. .68 Figure 4-4: Various structures attained by GLAD plus rotation and deposition control. (a) Vertical pillars, (b) inclined pillars, (c),(d),(e) variable diameter pillars, (f) fractal like structures, (g),(h) columns with capping layers, (i) helix, (j) waved columns, (k) zig-zag, (l) zig-zag pillar with variable diameter (63). .68 
Figure 4-5: GLAD fixture from TEMESCAL for e-beam evaporator BJD 2000, also from TEMESCAL.

Figure 4-6 : (left) Electron beam evaporator, Ion beam assisted deposition equipment, and Glad fixture; (right) planetary was completely removed and replaced it with GLAD apparatus.

Figure 4-7: Profiles of flux angle variation in time with different values of $\mathrm{B}$ on the exponential

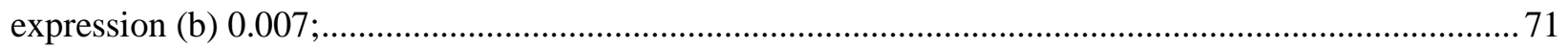

Figure 4-8: SEM micrographs of final capping layer for each profile proposed in Figure 4-7 ................72 Figure 4-9: Profiles of the main deposition parameters for GLAD: (a) flux angle $[\alpha=100 * \exp (-0.028 * t)]$, (b) deposition rate, (c) rotation speed of the substrate. $\mathrm{t}=20 \mathrm{~min}$ is the time when the exponential decay of the flux angle started .73

Figure 4-10: SEM micrographs of final capping layer on top of PhC structure (total thickness 320nm) 74

Figure 5-1: Process flow for fabrication of Photonic crystal slab on Si .76

Figure 5-2: SEM micrographs of fabrication process of a PhC slab on Silicon: (a) cross-section of $\mathrm{PhC}$ on ma-N 2403 resist; (b) top-view of PhC slab on Si plus nanocavity defect; (c) $45^{\circ}$ view of many suspended $\mathrm{PhC}$ slabs on SOI; (d) cross-section of a final suspended PhC slab on SOI.

Figure 5-3: schematic diagram of experiment for characterizing light enhancement inside the $\mathrm{PhC}$ nanocavity 


\section{LIST OF TABLES}

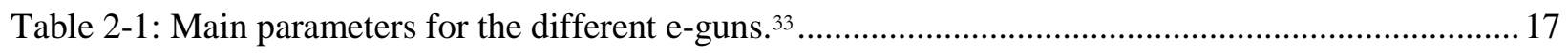

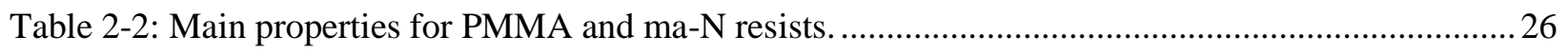

Table 3-1: Lattice parameters for PhC patterning on a negative resist using GaN substrate.....................42

Table 3-2: Growing parameter for PECVD and E-beam evaporation .................................................. 47

Table 3-3: Etching rates for $\mathrm{SiO}_{2}$ and $\mathrm{Ni}$ for different plasma chemistries. ......................................... 48

Table 3-4: Etching rates and selectivities for ICP etching of GaN in different plasma chemistries...........55

Table 4-1: Process parameters for GLAD deposition for optimization of capping layer ......................... 71 


\section{CHAPTER I}

\section{INTRODUCTION}

\subsection{PHOTONIC CRYSTALS}

Photonic crystals (PhCs) are special porous structures that are capable of periodically modulating the refractive index of a given dielectric material. These structures contain regularly repeating internal regions of high and low dielectric constant. These $\mathrm{PhC}$ structures can interact with electromagnetic radiation in the same way that periodic potential in a semiconductor crystal can alter the electron motion. Photons, which can behave as particles or waves, may or may not propagate through these structures depending on their wavelength. Photon wavelengths that are allowed to go through these engineered structures are known as modes, and groups of allowed modes are called bands. On the contrary, group of modes that cannot make it through these structures are known to form photonic band gaps. Figure 1-1 (b) illustrates a dispersion diagram which contains all the allowed and prohibited bands in the structure along different directions. This effect, that $\mathrm{PhC}$ structures have, is mainly based on diffraction; consequently, the periodicity of the $\mathrm{PhC}$ structure has to be of the same order as half the wavelength of the electromagnetic radiation traveling through the structure. Changes in dielectric periodicity on the order of $\sim 200 \mathrm{~nm}$ to $\sim 350 \mathrm{~nm}$ would be small enough to operate in the visible part of the electromagnetic spectrum. A PhC structure that may exhibit a photonic bandgap is shown in Figure 1-1 (a). ${ }^{1-4}$

A photonic bandgap enables photonic crystals to be able to guide or localize light with very low losses or to inhibit spontaneous emission. ${ }^{5}$ To control the behavior of light, the periodicity of the dielectric constant can be arranged in one, two or three spatial dimensions. It has been demonstrated that only 3D PhC structures present complete bandgap in all directions and any polarization. ${ }^{6}$ However, the precise nanofabrication of 3D structures using current technologies represents a major challenge. Theoretical studies have found that some 2D PhC structures have many properties similar to those of the 3D ones. 2D structures with a finite thickness in the vertical direction are known as PhC slabs. Such 2D structures are more practical and easier to fabricate. 2D PhC slabs are structures that have the field confined in a two-dimensional plane in such a way that only one mode (or two if polarization are considered) exists in the direction normal to the plane. Optical modeling has been developed to engineer 
the location and size of the photonic band gaps (PBG) of PhCs, and it may be based on the following methods:

a) The plane wave expansion method.

b) The finite Difference Time Domain method.

c) The order-N spectral method

In these $\mathrm{PhC}$ slabs, one can introduce one or more line or point defects that locally interrupt the periodicity of the lattice structure. In the defect a localized mode may exist with a frequency inside the photonic bandgap, which can be strongly confined to the region of the defect due to the surrounding lattice. A point defect in a $\mathrm{PhC}$ structure is known as a nanocavity. ${ }^{7}$ This defect is intentionally introduced and it allows the propagation of a single mode through the whole structure. As a result, a relative sharp peak appears in the transmission or reflection spectrum and in a dispersion diagram a single mode appears in between the photonic bandgap. This electromagnetic confinement produced by the nanocavity is measured by a parameter Q called the quality factor which measures how strong the cavity can confine light. ${ }^{8-10}$ Finite difference time domain is a method that is used to design and simulate PhC structure with a specific Q factor. Figure 1-1 (d) displays a 2D PhC structure slab along with a cavity in the center.

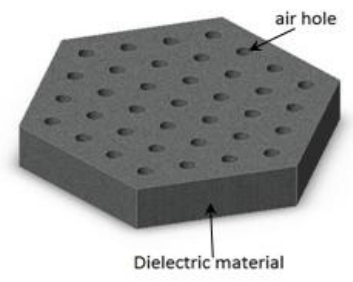

(a)

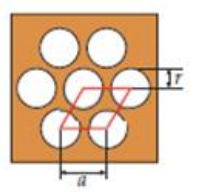

(b)

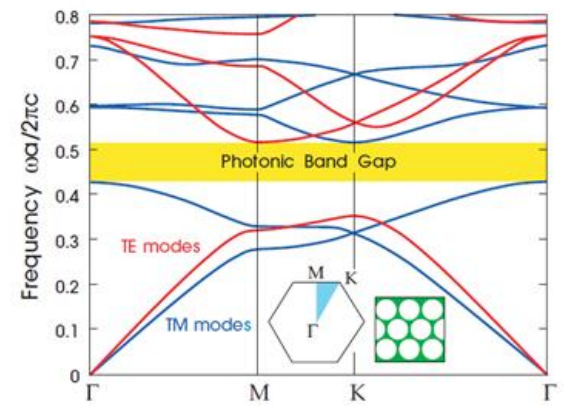

(c)

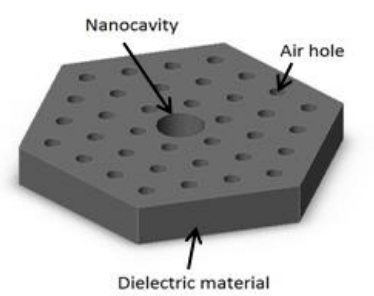

Figure 1-1: (a) a 2D Photonic Crystal slab structure, (b) dimensions to be considered in a PhC structure, (c) dispersiondiagram of a hexagonal lattice $\mathrm{PhC}^{4}$, (d) a point defect introduced in the center of a PhC structure. 


\subsection{PhC FOR BIOSENSING APPLICATIONS}

\subsubsection{Biosensors}

Based on fact that there are many different fields where biosensors can be applied and principles that these devices use for detection, it can be said that there is a huge need for gathering expertise from the distinct areas of science and industry to collaborate with each other for the successful fabrication of biosensors. Figure 1-2 illustrates the entire arena required for the biosensor development.

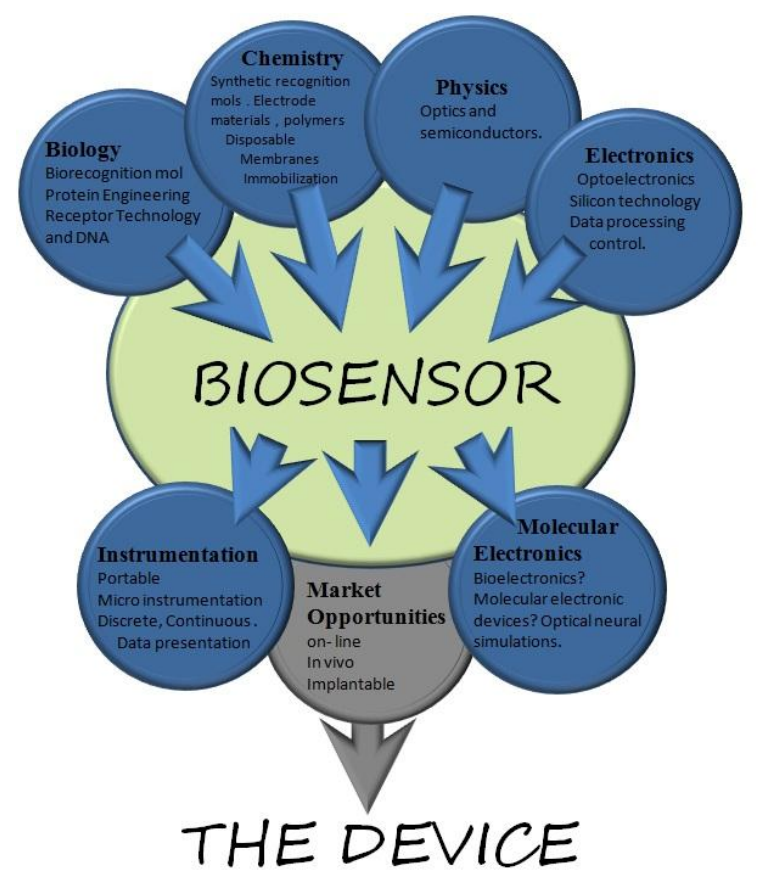

Figure 1-2: Multiple expertise put together to achieve successful biosensors. 11

Nowadays, there are a vast variety of commercially available biosensors that are capable of high precision detection of specific biomolecules in an analyte, kinetic binding constant measurements and high resolution imaging of biomolecules. Even though they vary in the transducing principle and application, they share some properties that are enormously important for the biosensor to be fully accepted in commercial markets, including: ${ }^{11}$

- Relevance of output signal to measurement environment

- Accuracy and repeatability 
- Sensitivity and resolution

- Dynamic range

- Speed of response

- Insensitivity to temperature (or temperature compensation)

- Insensitivity to electrical and other environmental interference

- Amenable to testing and calibration

- Reliability and self checking capability

- Physical robustness

- Service requirements

- Capital cost

- Running costs and life

- Acceptability by the user

- Product safety

A Biosensor can be described as a device that attaches a biological sensing element, close or placed together, with a transducer (element that generates a measurable signal that could be electrical, thermal or optical) to form a sensing system for a specific analyte. The biosensor relies on an immobilized biological element, which makes the device uniquely sensitive to a specific target species. The transducer generates a signal based on the interactions between biorecognition molecules (biological sensing element) and the analyte. This approach is considered as an indirect assay to detect chemically similar species to those immobilized on the biorecognition layer. The species can be DNAs, enzymes, antibodies, nucleic acids, immune systems, tissues or whole cells. Figure 1-3 shows how analyte recognition works and the biosensor principle. ${ }^{11}$
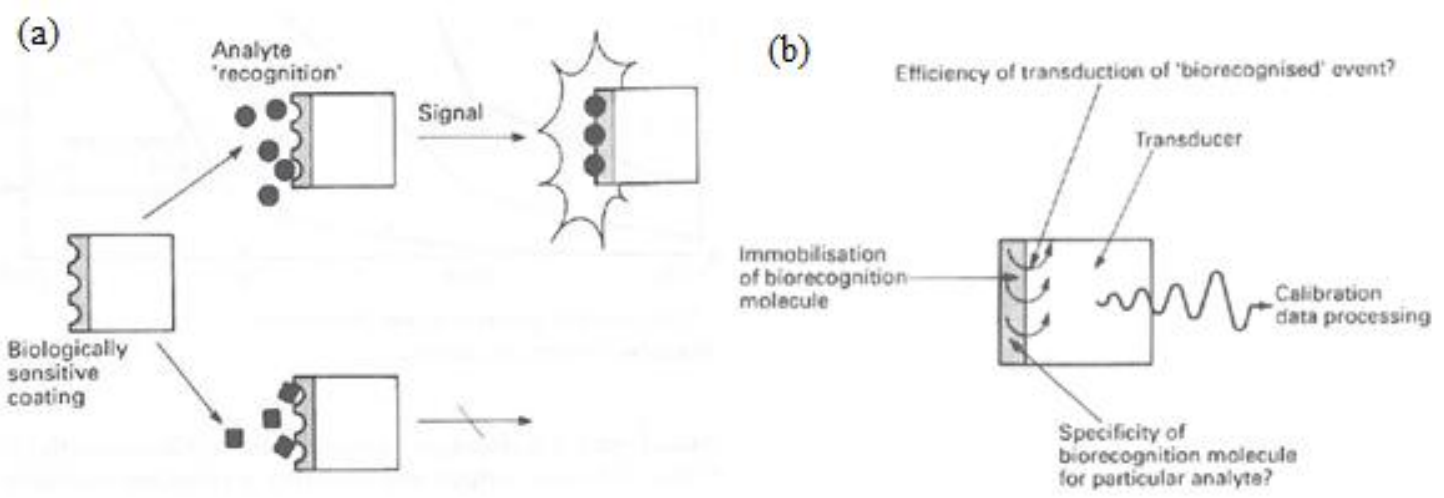

Figure 1-3: Biosensor: (a) surface modified-transducer, reactive to specific analyte, (b) principle of operation of a biosensor in general. ${ }^{11}$ 
Biosensors can be classified according to the transduction method as optical and electromechanical. Because electromechanical biosensors are out of the scope of this thesis only optical biosensors will be reviewed. Optical biosensors have demonstrated excellent properties for analysis and detection of biomolecules, and the associated sensing methods have proved to function very well in an ample range of fields because of its immunity to magnetic interferences, capability to biosensing at remote levels, and multiplexed detection for a single assay.

Optical biosensors can be classified as fluorescence-based biosensing and label-free biosensing. Fluorescence-based biosensor consists of labeling a tag biomolecule with fluorescent dies commercially purchased; the fluorophore attached to the biomolecule causes the biomolecule to become fluorescent when excited with an external light source which confirms evidence of the presence of the tag biomolecule or interactions between biomolecules in an analyte. This method is extremely sensitive with the capacity of detecting up to single molecules.

Label-free detection does not use any kind of label, thus avoiding any kind of damage or contamination to the analyte. This allowed the biomolecule to be sensed in a natural way. Hence, this method becomes cheaper and easier to be carried out, and it still allows detection and kinetic analysis of biomolecules. Label-free detection is based on measurements of changes in refractive index, ${ }^{12,13}$ wavelength changes upon reflection, ${ }^{14,15}$ and Ramman scattering spectroscopy ${ }^{16}$ due to biomoleculebiorecognition layer interactions. These methods have demonstrated to be able to detect and image biomolecules in very low concentration (femtoliters to nanoliters) in an analyte.

Different fields call for different needs when it comes to biosensing, so both protocols, based on optical sensing, have been used extensively. However, label-free detection is becoming highly attractive because it simplifies assay work and cost. Lately, a great deal of biosensors has been designed based on label-free optical detection. An excellent description and its classification are explained by Fan et. al ${ }^{17}$. Many types of optical biosensors are constructed based on PhC structures, and will be briefly described in the following sections.

\subsubsection{Photonic crystal waveguide biosensors}

These devices are based on planar photonic crystals membranes, which are also known as planar photonic bandgap components. Light is guided along defects to obtain very high and spatially selective changes in the RI of the surroundings; in this case, the defect is missing rows of holes or rods on the entire structure (photonic crystal waveguide). Figure 1-4 (a) shows the photonic crystal waveguide structure on Silicon on insulator. 
The basic property of a $\mathrm{PhC}$ waveguide is that a given bandwidth of light can be guided in the waveguide as the light is confined laterally by the PC and vertically by total internal reflection (TIR). The dispersion characteristics of this waveguide are such that it is effectively single-mode at low frequencies, with a cut-off falling inside the band-gap. This mode cut-off causes an abrupt drop of the output power in the transmission spectrum. Adding a thin surface film modifies the local refractive index, changing the effective refractive index of the slab and the refractive index contrast between the 'hole' and 'slab' regions. The device can thereby be used as a sensor by monitoring the cut-off wavelength shift resulting from the attachment of the target on the sensor surface; Figure 1-4 (b) shows the power transmission spectrum of the biosensor for different solutions and their different cutoff wavelength shift.

(a)
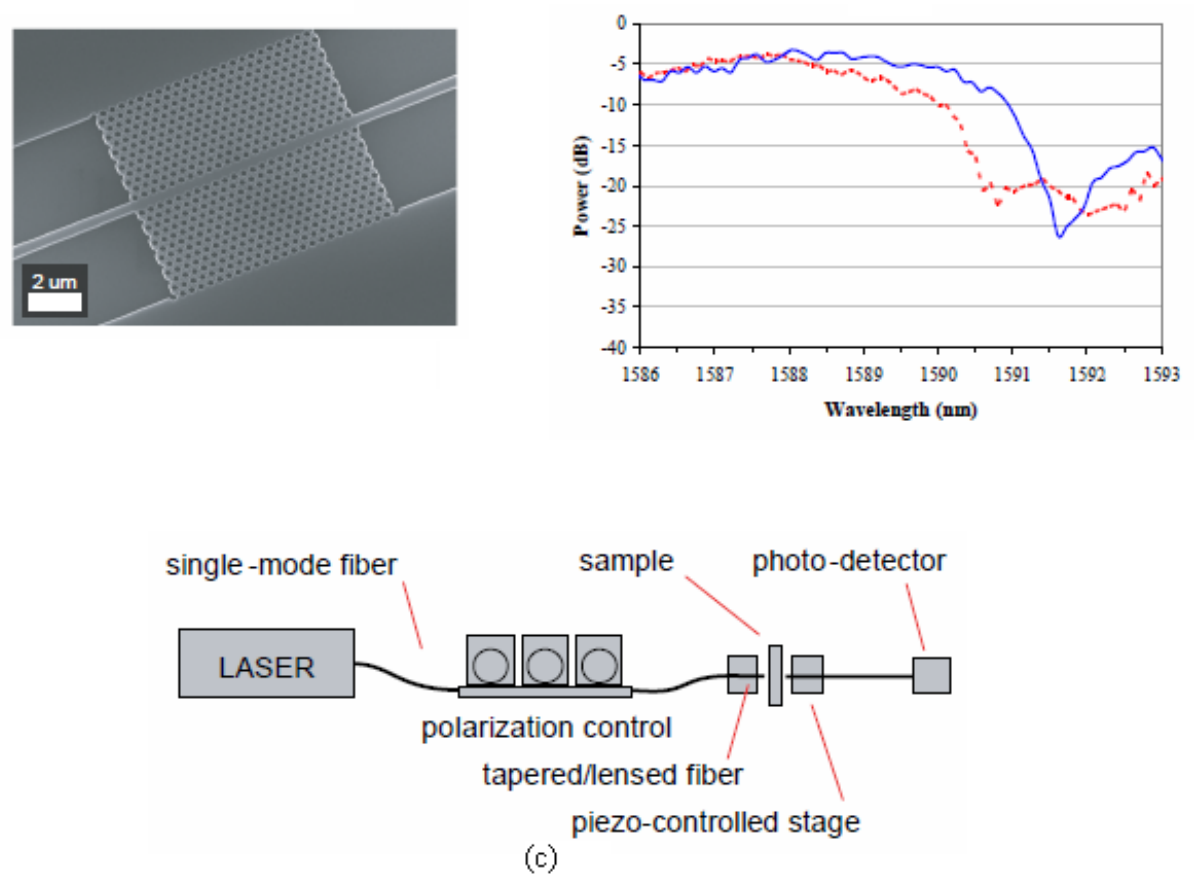

Figure 1-4: (a) PhC waveguide slab on SOI; missing row of holes constitue the waveguide, (b) transmission spectrum showing shift s of the cutoff wavelength on the structure, (c) setup for the whole biosensing technique. ${ }^{18}$

Specific biosensing relies on immobilized biological receptors which bind selectively to the target analyte. In the case of refractive index biosensors, this binding event increases the local refractive index, which consequently alters the cut-off wavelength on the transmission spectrums. A PhC waveguide biosensor has a large sensing range of RI measurements going from air to high viscous fluids. This device has been able to perform RI-measurements and protein detection. A good property of this biosensing 
technique is that the setup can be made compact and integrated with both additional optical and electronic components onto one single chip, as seen in Figure 1-4 (c).

Upon the solution being placed ( $\mu$ volume) on top of this device (no labeling needed), the transmission spectrum is measured and compared to the one obtained for water on top of the waveguide, thus the variation of RI is determined. It has been demonstrated that $\mathrm{PhC}$ waveguides can also detect different surface densities of analyte. This sensor has a logarithmic response to changes in cutoff wavelength vs. protein-concentrations which demonstrates its capability to measure up to $6 \mathrm{ng} / \mathrm{mm}^{2}$ of analyte and bulk response of $88 \mathrm{~nm} / \mathrm{RIU} .^{18}$

\subsubsection{Photonic crystal fiber biosensors}

This type of $\mathrm{PhC}$ biosensor is based on PhC fibers, which are fiber optic cables with air holes specially arranged in the cross-section, as seen on Figure 1-5 (a). PhC fibers have a periodic triangular air hole structure and a core formed by a defect consisting of a missing air hole. The fibers are characterized by the hole size and the relative free-space wavelength. PhC fibers are usually made from pure silica ( $\mathrm{SiO} 2$ which is biocompatible and chemically inert) or polymer materials (polymer materials allow a wide range of surface chemistries)

In these fibers the core mode is more confined to the silica material than the cladding mode, which has a larger portion of its field energy in the evanescent wave inside the holes of the fiber. If the refractive index in the holes is changed the effective index of the cladding mode will thus be more affected than the effective index of the core mode. In turn, this will be seen as a shift in the resonant wavelength. This is the principle that enables these structures to be used in biosensing application.

As with the $\mathrm{PhC}$ waveguide biosensor, $\mathrm{PhC}$ fiber biosensors need Poly-L-lysine to immobilize negatively charged molecules such as DNA to a solid support. Poly-L-lysine has positively charged amino-groups that bind to the negatively charged silica surface through an ionic binding. DNA (deoxyribonucleic acid), on the other hand, has negatively charged phosphate groups in its backbone, and thus may be immobilized in a monolayer onto the poly-L-lysine. A pressure chamber is used to introduce the analyte in the holes of the fiber and then any excess of analyte is washed. Then the fiber is plugged into a light source in order to record the transmission spectrum of the fiber. Figure 1-5 (b) illustrates a single hole and the biofilms (PLL and DNA) on the inside of the walls. 
(a)

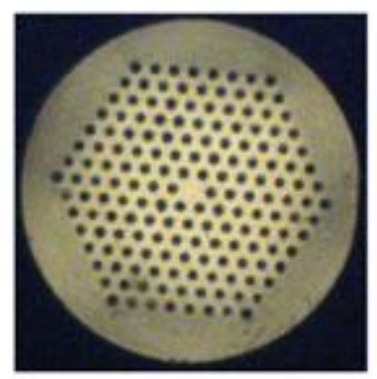

(b)

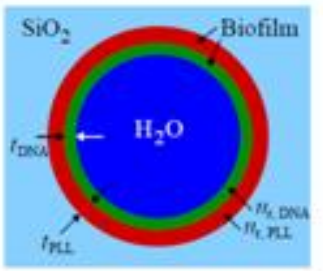

(c)

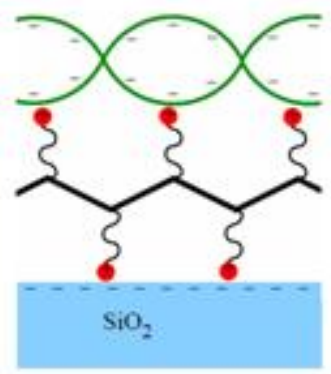

Figure 1-5: (a) cross section of PhC fiber with a defect in the center, (b) biofilms attached to the inside of each hole in the fiber, (c) The biomolecular structure attachment of poly-L-lysine (black and red) immobilized onto the silica surface ( $\mathrm{SiO} 2$ ), DNA(green) is immobilized on the poly-L-lysine. ${ }^{19}$

The measured transmission spectrum, as seen on Figure 1-6 (a), is used to find the peak of the transmission dip corresponding to the resonant wavelength of the $\mathrm{PhC}$ fiber with long period gratings. Shifts in the resonant wavelength on the order of 2-3 nm have been detected, Figure 1-6 (b) shows these resonances wavelengths and their respective shifts. This biosensor has demonstrated to be very sensitive with a resolution of $10^{-4} \mathrm{RIU}$. PhC fiber biosensing has become a promising label-free sensing platform for two reasons: first, the air holes in the fiber can act as fluidic channels to carry the analyte to be studied; second, the exclusive light guiding mechanism of $\mathrm{PhC}$ fibers ensures a strong light-matter interaction. Air holes in the fiber may hold a sample volume of a few $\mathrm{nL} / \mathrm{cm}$; therefore this technique does not need a large amount of analyte per assay, making it desirable for biosensing applications. $\mathrm{PhC}$ fibers along with long period gratings have proved well in detecting average thickness of layers of biomolecules within a few $\mathrm{nm}$. It is robust, simple and insensitive to temperature fluctuations and it does not require any calibration of the optical setup; however, it is not able to detect single molecules.
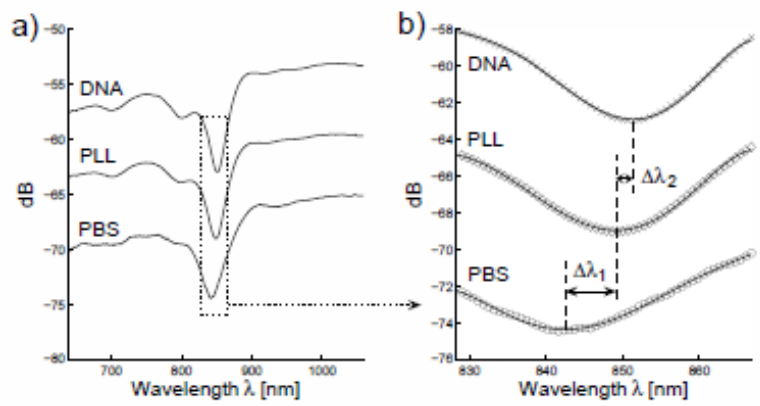

Figure 1-6: (a) transmission spectrum for PBS (phosphate buffered saline solution), PLL (poly-L-lysine) and doublestranded DNA (b) close up of dotted rectangle in (a). 19 


\subsubsection{Photonic crystal nanocavity biosensors}

A two-dimensional photonic crystal nanocavity biosensor, as illustrated in Figure 1-7 (a), is constructed by introducing a defect in an otherwise perfectly periodic structure. This defect can have a radius smaller or larger than the one of the rest of the $\mathrm{PhC}$ structure. An optical biosensor based on light propagation needs to couple light and direct it to the $\mathrm{PhC}$ structure toward the nanocavity. This allows light to interact with matter (analyte) and alter the refracting index which causes shifts in the allowed mode of the structure. Figure 1-7 (a) displays the suspended PhC structure on SOI with ridge waveguides at the ends to guide light in and out of the structure

In biosensing applications, the entire defect internal surface is functionalized with probes (e.g., antibodies), thus, the target virus can be immobilized at the defect center or in the vicinity of the sidewall; the presence of molecules inside the central hole and the first layer of surrounding holes will cause a local RI changes, Figure 1-7 (b) shows a transmission spectrum without and with a biomolecule inside the defect. A light beam is coupled into the input ridge; the transmitted signal is then coupled out in a similar fashion and measured using a detector to record the spectral response to the capture of biomolecules in the defect. Figure 1-7 (c) displays the whole setup for this biosensing technique. Such a configuration also gives rise to a resonance in the cavity with a relative low quality factor. This defect can make the field concentration large enough to make the device very sensitive to small refractive index changes which can be monitored as the spectral shift of the resonant wavelength of the $\mathrm{PhC}$ nanocavity.

The $\mathrm{PhC}$ biosensor has a sensing area of $\sim 40 \mathrm{~m}^{2}$, thus a very small amount of analyte is required per assay. Moreover, this is structure can be made using mature microelectronic fabrication techniques, which makes it better suited for integration with electronic/photonic components on a single chip. These devices have demonstrated to be capable of detecting 0.002 ambient RI changes with a passive $\mathrm{PhC}$ nanocavity with relative low $\mathrm{Q}$ factors. The minimum amount of protein that can be detected by the device is $\sim 2.5 \mathrm{fg}$. However, if we are able to precisely deliver the targets to the defect area only, the sensitivity could be further improved to the single bio particle detection level as long as the biomolecules are greater than $50 \mathrm{~nm}$ in diameter. ${ }^{20}$ 


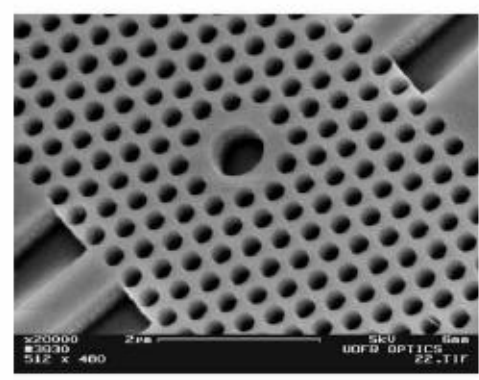

(a)

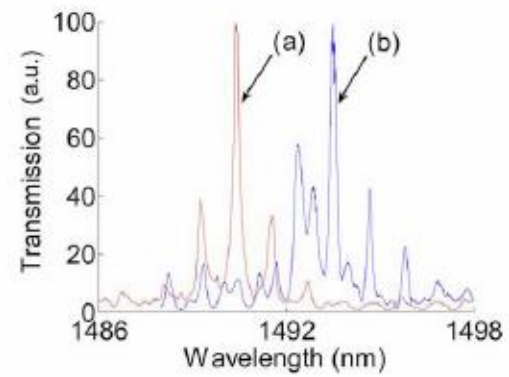

(b)

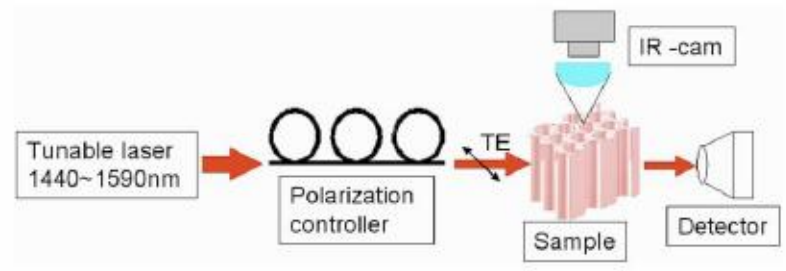

(c)

Figure 1-7: (a) top view of the $\mathrm{PhC}$ with the defect and its ridge waveguides at the ends, (b) transmission spectrum for such structure, (c) schematic of the biosensing setup. ${ }^{20}$

The trend of biosensing device fabrication is to elaborate a single chip that would be able to analyze biomolecules and turn in results immediately, so called Lab-on-a-chip. ${ }^{21}$ These hand-held devices integrate light sources, sensors and detectors along with microfluidic channels for analyte supply, and an on-board optical and electronic controls, which might be light filters or signal filter processors used to provide results that only dedicated labs have been able to do.

\subsection{OPTOFLUIDIC PHOTONIC CRYSTAL RESONATOR SENSOR}

The integration of microfluidics and optics represent a new emerging field that has strongly influenced biosensing technology, as reviewed by Psaltis et. all. ${ }^{22}$ It has been demonstrated that $\mathrm{PhC}$ cavities in biosensors are able to confine light in extremely small volumes. This greatly increases light matter interactions, and habilitates measurements of analytes in the orders of femtoliters. ${ }^{23}$ Fluids at micro level have been studied extensively and their understanding is vital when this converges with optics; fluid properties can alter optical properties (optofluidics). All the technological advances in microfabrication have made possible the fabrication of tiny structures such as pumps and valves that are connected with very complicated channel networks allowing control over the microfluid. 
The present work aims to integrate $\mathrm{PhC}$ nanocavities with optofluidic channels to fabricate a portable and highly sensitive biosensor. Label free biosensors have excellent characteristics due to their ease to perform an assay. However, they present some drawbacks such as once the device has been used it needs to be disposed because immobilized particles in the structure cannot be removed completely when washed. The residues may provoke issues on a second assay. The proposed device is label-based which does not need any biorecognition layer in the structure to detect target molecules which lets the device be washed and reused for numerous assays. A high sensitive is expected due to the analyte labeling. Multiple device fabrication in a single substrate can allow parallel operation, improving in this way throughput because many assays can be performed at same time. The architecture and operation of the sensor are described in detail in ${ }^{24}$ and briefly summarized here. Figure 1-8 is a schematic illustration of the sensor operation principle. The operation can be divided into several steps that are described below.

(a)
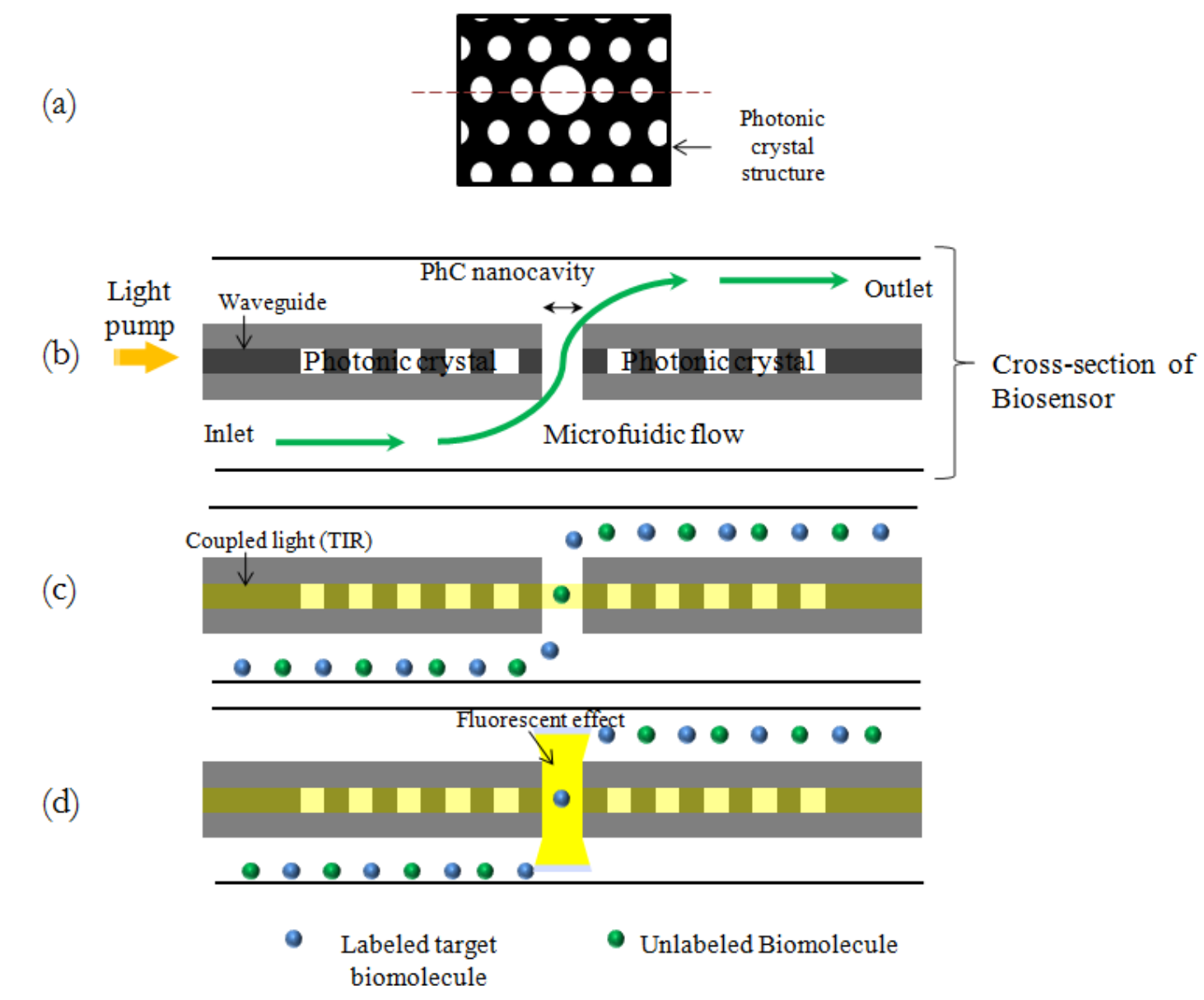

Figure 1-8: (a) Top view of an optofluidic PhC resonator biosensor; (b) cross section schematic of the biosensor; (c) light enhancement is produced by the cavity due to the presence of labeled biomolecules; (d) no light enhancement is produced by unlabeled biomolecule even when they are in the cavity. 
First, the analyte is dyed with the appropriate fluorophore. The fluorophore should have an excitation frequency that is allowed to propagate through the $\mathrm{PhC}$ structure, and its emission frequency should lie within the Photonic bandgap in order to generate light enhancement due to the presence of the nanocavity. It is very important to mention that fluorophores bind selectively with only target biomolecules in the analyte.

Then, the analyte solution will be introduced from a reservoir into microfluidic channels. The analyte will be driven and forced to go through the point defect in the PhC slab until solution reaches a waste reservoir. At the same time, light (with a wavelength not prohibited by the PBG) is coupled and travels through the waveguide to the PhC nanocavity.

Now, if light coming from the pump hits a labeled target biomolecule right in the cavity, the labeled target biomolecule will be excited and produce a fluorescence effect with a different wavelength than that of excitation. These photons emitted by the labeled molecule are not allowed to propagate in the $\mathrm{PhC}$ structure causing an enhancement of light in the cavity (resonance). The amplification of light improves the resolution of the sensor, making it possible to detect small concentration of target biomolecules in the analyte. Finally, a light detector will pick up this signal coming out from the cavity, and announce the presence of the target biomolecule in the analyte. Of course if any another unlabeled biomolecule goes into the cavity, it will not produce any fluorescence effect. Therefore, no fluorescence will be perceived by the detector. Figure 1-8 (c) shows that no light enhancement is present when an unlabeled biomolecule is in the cavity.

\subsection{THESIS ORGANIZATION}

The research work of this thesis is directed toward the development and optimization of nanofabrication of the optofluidic PhC resonator biosensor structures described above. This thesis is organized as follows: Chapter 2 reviews the key processes utilized in the nanofabrication of the above-mentioned structure, including E-beam lithography, thin film deposition and inductively-coupled plasma (ICP) etching. Chapter 3 describes the optimization of e-beam lithography and etching processes as well as the fabrication of $\mathrm{PhC}$ on $\mathrm{Si}, \mathrm{GaN}$ and ITO PhC structures.

Chapter 4 discusses the development of a recipe for sealing $\mathrm{PhC}$ structures by the glancing angle deposition technique. Uniform $\mathrm{SiO} 2$ capping layers are successfully deposited with minimal material extended down into the $\mathrm{PhC}$ airholes.

Chapter 5 describes the fabrication process of suspended PhC slabs on a SOI substrate and the use of the structures for resonance fluorescence characterization.

Chapter 6 summarizes the current research work and offers and outlook for future work. 


\section{CHAPTER II}

\section{NANOFABRICATION}

\subsection{INTRODUCTION}

Nanotechnology refers to the capacity to control and handle matter at nanoscale, thus this is responsible for designing, producing and characterizing devices with submicron dimensions. ${ }^{25}$ Therefore, Nanotechnology develops methods that allow the fabrication and study of materials considering atom, molecule and particle behavior. ${ }^{26}$ These studies most of the time require attaching to a substrate or patterned areas of a substrate. Fabrication at small scale has being carried out by two important methods: top-down technique and bottom-up technique. ${ }^{27}$ The former means that control of shape and size is made from bulk material to fabricate the small structures that are desired, the latter deals with the assembly of fundamental matter units to form large structures. Some examples for top-down approach are lithography, soft lithography and evaporation techniques, and for bottom-up approach are supramolecular assembly, ${ }^{28}$ monolayer self assembly ${ }^{29}$ among others. Lately, nanotechnology has been trying to integrate both methods to develop new hybrid techniques habilitating nanofabrication in 3D.

These new technologies for nanofabrication have the main goal of shrinking dimensions in order to improve speed and reduce power consumption in electronic devices and to increase also the number of the devices in a substrate fabricated. As a result, devices costs are decreased whereas quality and performance is improved. However, as shrinking is pushing further, limitations due to quantum effect are more dominating demanding more and more efforts to maintain Moore's law keep going. ${ }^{30}$

It is worth to mention that fabrication processes are to be realized with a high precision and resolution; therefore, environmental conditions are excessively important to be controlled. Then, all these nanofabrication processes are performed in special facilities called Cleanroom; these facilities are designed to keep low levels of particles in the air, acoustic noise and electromagnetic interference. At the same time, control temperature and relative humidity should be kept at standard levels, inside these facilities. 
This work has been focused on top-down fabrication, consequently, E-beam lithography. E-beam evaporation and ICP etching processes will be discussed.

\subsection{ELECTRON BEAM LITHOGRAPHY}

Optical lithography has been the most popular technique in microelectronic fabrication, because of its ease to be used, high throughput and compatibility with $\mathrm{Si}$ processes, when employed in the semiconductor industry. Extensive efforts have been done to improve the resolution replacing near UV sources for deep UV or x-ray sources but the former increased cost and the latter had to deal with the fact that $\mathrm{x}$ rays can penetrate metal masks, thought fine features as small as $200 \mathrm{~nm}$ have been reported with

this approach. ${ }^{31}$ Nowadays, it is believed that this method cannot improve resolution any further because of light diffraction phenomena (Rayleigh scattering). Then, non optical based lithographic methods have been necessary to be implemented to scale down features to sub-100nm scale. Early 1980's, charged particle beam lithography was the focus of many researchers as a solution to overcome the light diffraction issue in photolithography.

This new method of lithography comprises electron beam and ion beam lithography, due to the availability of the first system, focus will be placed on it. Electron beam lithography (EBL) is a technique based on a high energy electron beam specially designed for elaborating quite fine features. Basically, this kind of lithographic method scans a pulsing electron beam from top to bottom and left to right across an area previously coated with a substance sensitive to electrons called resist, in this way the electron beam deposits energy in the required pattern in the resist film. Some advantages and disadvantages of this technique are the following:

- Maskless process

- High resolution capability

- Compatibility to be used in any material

- Low throughput

- Surface damage

- Costly and complex tool

Dedicated EBL systems are used in the industry but they are pretty expensive ( $>1 \$$ million) due to their special features like high accelerating voltages (up to $100 \mathrm{kV}$ ), laser based stages and proximity effect correction tools. For research projects usually an Scanning electronic microscope (SEM) is converted into EBL by means of NPGS or RAITH or Nanomaker systems which are software hardware 
packages that let the user design patterns, calibrate the system and control the placement of the beam and the exposure execution. Figure 2-1 shows a SEM based EBL system from JOEL.

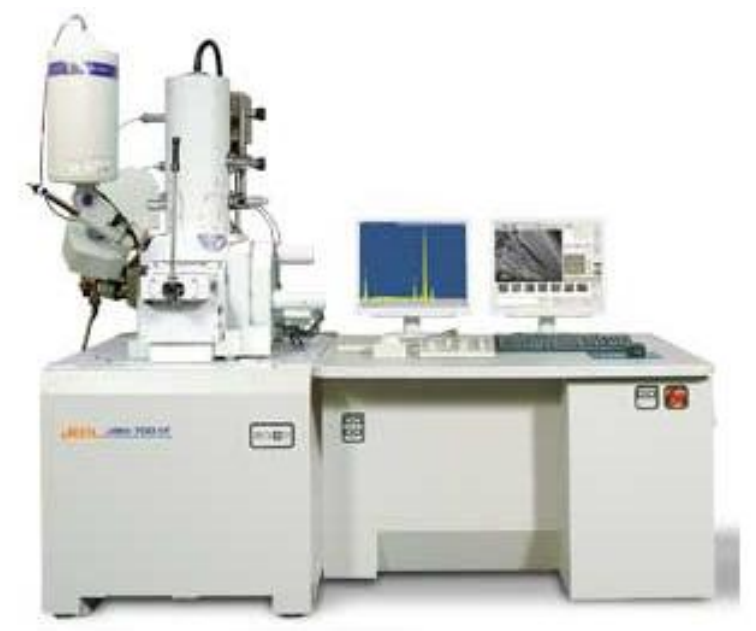

Figure 2-1: SEM based electron beam lithography. ${ }^{32}$

\subsubsection{Architecture}

The process of EBL itself is relatively simple; however, the physics of its parts are pretty complex. Even though understanding how some of the components of EBL work is demanding for determining the system limitation, thus optimization of the writing process is possible. Figure 2-2 shows the main parts of an SEM system that can be divided into: electron source, column and chamber under vacuum, electron detector, and stage.

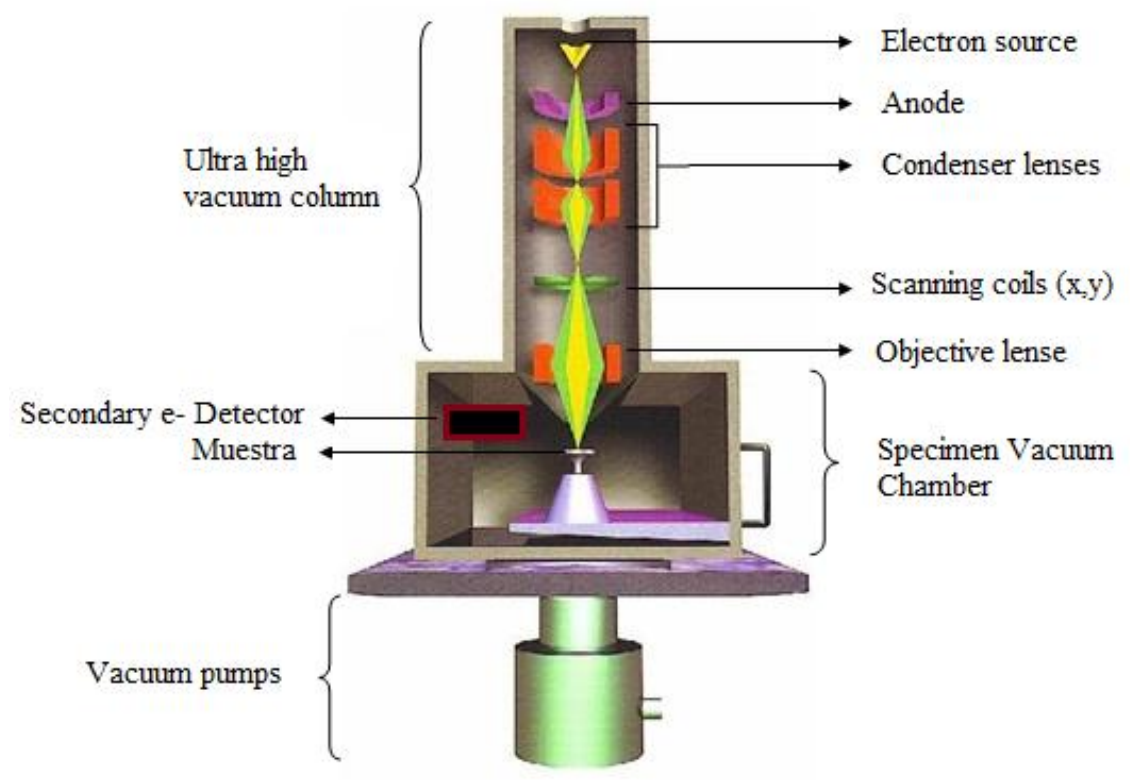

Figure 2-2: Parts of an SEM system. 


\subsubsection{Electron sources}

The electron source is the heart of an EBL system, and its main purpose is to generate an electron beam in a particular direction. Electron sources are based on different mechanisms to generate the emission current; these methods include:

Thermionic emission - This is caused by heating up the filament, so that electrons gain enough energy to surpass the work function barrier of the conductor. For years, this source has been used in EBL systems, and it was a simple loop of tungsten wire where a current was passed through to generate the heat. This kind of source has very poor brightness and high spread energy because it works at very high temperatures. Later on, lanthanum hexaboride $\left(\mathrm{LaB}_{6}\right)$ was discovered and it proved better brightness and lower spread energy. 33

Thermal field emission - This type of electron gun uses an electric field to generate the emission current and this takes place when $\mathrm{ZrO}_{2}$ is supplied to the $\langle 100\rangle$ surface of the tip which is made of tungsten (W), this causes the lowering of work function of $\mathrm{W}$. Also the tip is heat up to $1800 \mathrm{~K}$ to be supplied with the $\mathrm{ZrO}_{2}$ constantly. This has proved great to keep a very stable and high probe current for EBL systems, thus this source presents high brightness; small spot size and low spread energy. ${ }^{33}$ Figure 2-3 show a schematic of this type of source.

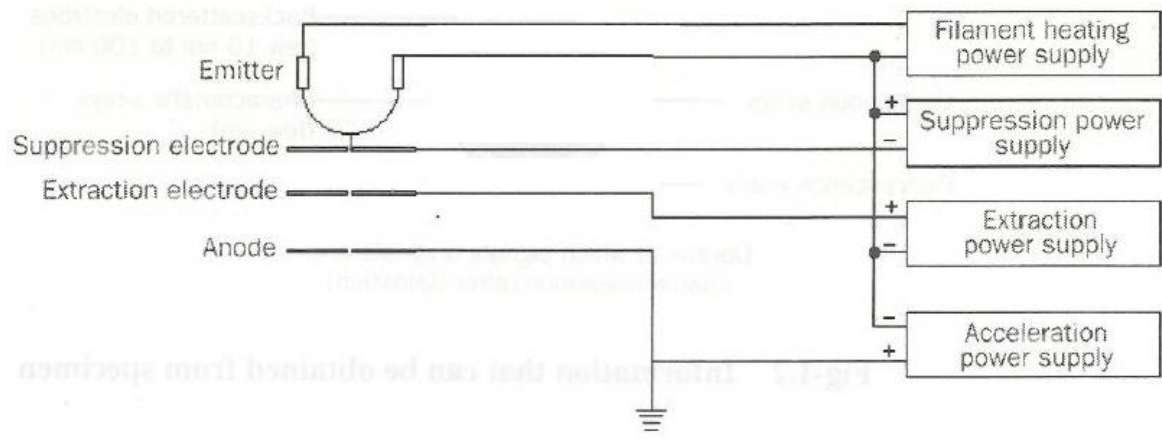

Figure 2-3: Field Emission Electron Gun (JEOL system). ${ }^{32}$

Cold field emission - This type of source works at room temperature and the electron extraction is due to tunnel effect from the surface of the tip when a strong electric field is applied to the surface $\langle 310\rangle$, this cathode is usually made of tungsten. This electron source has higher brightness and smaller proved current than the thermionic source although the use of this electron gun has become uncommon in EBL 
systems because of its instability due to short term drift and long term drift, which turns out being unacceptable for EBL but for imaging (electron source used in TEM systems). Changes in work function of the tip due to atoms being absorbed onto its surface are the main reason for such noise which consequently affects the emission current. This issue is reduced if the tip is heated up for a short period of time to clean it. Nevertheless, new atoms or molecules can be readsorbed even in very high conditions of vacuum. Also atoms might be ionized by the electron beam and accelerated back into the tip what causes sputtering effects on the tip. Due to these serious issues which cause prove current fluctuations, cold field emission sources are housed in chambers at ultra high vacuum. ${ }^{32}$ Table 2-1 displays a summary of the main parameters of the different sort of electron guns available on the market.

\begin{tabular}{lcccccc} 
Source type & $\begin{array}{c}\text { Filament } \\
\text { material }\end{array}$ & $\begin{array}{c}\text { Brightness } \\
{\left[\mathbf{A} / \mathbf{c m}^{2} / \mathbf{r a d}\right]}\end{array}$ & $\begin{array}{c}\text { Energy spread } \\
{[\mathbf{e V}]}\end{array}$ & $\begin{array}{c}\text { Vacuum } \\
\text { required } \\
{[\text { Torr] }}\end{array}$ & $\begin{array}{c}\text { Spot size } \\
{[\mathbf{n m}]}\end{array}$ & $\begin{array}{c}\text { Filament } \\
\text { temperature } \\
{[\mathbf{K}]}\end{array}$ \\
\hline $\begin{array}{l}\text { Thermionic } \\
\text { source }\end{array}$ & $\mathrm{W}$ & $\sim 10^{5}$ & $2-3$ & $\sim 10^{-6}$ & $\sim 25000$ & $\sim 3000$ \\
\hline $\begin{array}{l}\text { Thermal } \\
\text { field } \\
\text { emission }\end{array}$ & $\mathrm{Zn} / \mathrm{O} / \mathrm{W}$ & $\sim 10^{8}$ & 0.9 & $\sim 10^{-9}$ & $\sim 20$ & $\sim 1800$ \\
\hline $\begin{array}{l}\text { Cold } \\
\text { emission }\end{array}$ & $\mathrm{Z}$ & $\sim 10^{6}$ & $2-3$ & $\sim 10^{-8}$ & $\sim 10000$ & $\sim 2000-3000$ \\
\hline
\end{tabular}

Table 2-1: Main parameters for the different e-guns. 33

\subsubsection{Electron optics}

After electrons are generated from the gun, it is required to make them converge to a specific axis and plane. In light optics, light is focused by optical lenses; here electrons can be focused by electrostatic lenses or magnetic lenses. Magnetic lenses present less aberration levels than the electrostatic lenses; as a result magnetic lenses are used in electron beam columns. A magnetic lens is a device that can focus or deflect the electron, so they converge or diverge for different purposes. Electrons are charged particles that obey electromagnetic laws. Coulomb and Lorentz laws can describe the force applied to electrons when they interact with an electric or magnetic fields, respectively. Magnetic lenses are compound of a coil, with a current running through it, which is capable of generating a magnetic field that interacts with the flux of electrons coming from the gun. These magnetic lenses are in charge of determining final size, 
shape and current density on the electron beam. Figure 2-4 exhibits a basic configuration of a magnetic lens and its interaction with an electron.

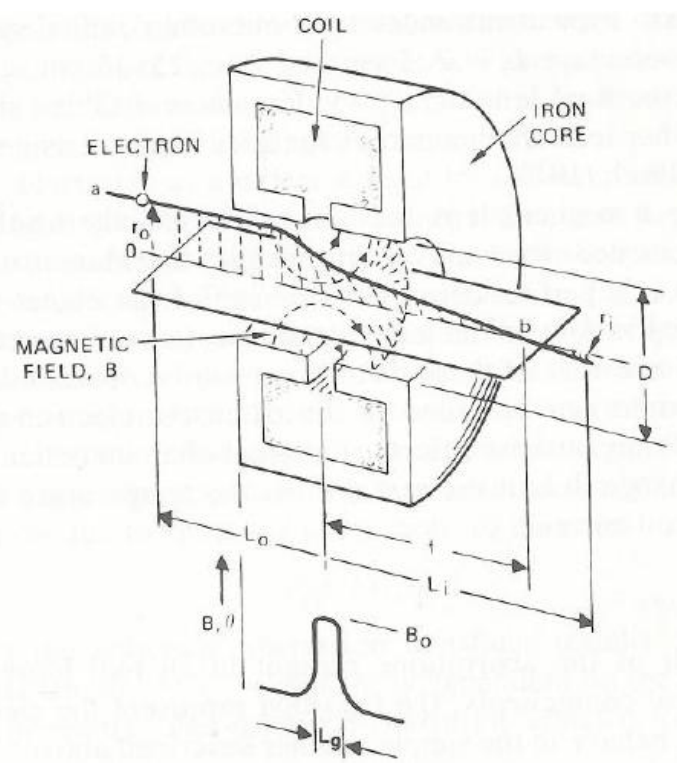

Figure 2-4: Cross-section of a magnetic lens. ${ }^{34}$

This magnetic lens is basically a coil with an iron nucleus that carries a current (probe current). This current creates an approximately uniform magnetic flux density over the gap Lg. Any electron running into this lens will interact with the radial component of the magnetic field what makes the electron to rotate. This interactions cause the electrons to focus inwardly, in a focus plane located along the axis and at a distance $\mathrm{f}$, when no aberrations are present (ideal lenses). Aberrations are present in real magnetic lenses and they are linked to changes in magnetic or electric field. These changes can be created by electronic reasons like: power supply variation, coil current variation, stray magnetic fields or by mechanical reasons like: apertures not perfectly rounded or tilted lenses.

Aberrations in the magnetic lenses affect the beam spot (bigger radius) and the focus point. An important type of aberration on this kind of lenses are spherical aberrations, where the outer zones of the lenses focus more strongly than the inner zones and it is given by the following equation:

$$
d_{s}=\frac{1}{2} * C_{s} * \alpha_{i}^{3}
$$

Where $d_{S}$ is the disk of least confusion, $\alpha$ is the beam convergence angle, $C_{S}$ is spherical aberration constant, this constant is function of lens excitation, lens geometry and paraxial focal length.

Another important type of aberration is chromatic aberrations, which have to do with the fact that some electrons have different energies so that they focus at different focal lengths. Lorentz force can 
explain how a moving charged particle is affected in a magnetic field depending on the particle velocity $\left(\mathbf{F}=\mathrm{e}^{-} \mathbf{v} \times \mathbf{B}\right)$, what results in variation of the focal properties of the lens. The next equation shows the disk of least confusion for this kind of aberration.

$$
d_{c}=\frac{\Delta V}{V o} * C_{c} * \alpha_{i}
$$

Where $\Delta \mathrm{V}$ is the energy spread of the beam, $\mathrm{V}_{\mathrm{O}}$ is the mean energy of the electrons on the beam, and $\mathrm{C}_{\mathrm{C}}$ is the chromatic aberration constant, which depends also on the lens focal length, lens excitation and lens geometry.

One way to diminish the effect of this aberration of the magnetic lenses is to decrease the beam convergence angle, and then most of the electrons in the beam are confined to the center of the lens. This reduction on the convergence angle produces a decrease in the total beam current, therefore a trade-off exist between reducing aberration effects and resolution.

Down in the column, apertures (hole where the beam goes through down onto the target) are used to set the beam current in the column, to reject any stray electron in the beam, and to blank the beam when the system is not in use. These apertures also can reduce the angle of convergence, for limiting the beam current, hence reducing aberrations in the lenses; however, they can diffract the beam setting a limitation on the radius of the beam. This effect becomes really strong when the system works at its highest magnification. The least radius for the beam due to diffraction can be given by:

$$
\delta_{d}=\frac{0.6 * \lambda}{\alpha}
$$

Where $\lambda=\frac{12.25}{\sqrt{V_{o}}}$ [Angstroms] is the electron wavelength in quantum mechanics, this wavelength is still much smaller than the wavelength of light, that is why this system has better resolution than UV photolithography.

Resolution in these systems is determined by taking into account all the effects just described previously: thermal transverse velocity effect, spherical aberrations, chromatic aberrations and diffraction. All these effects have been demonstrated to be statistically independent. Figure 2-5 is a plot that determines the beam radius for a system with a thermionic source. As seen on the plot, the smallest achievable beam radius is obtained at an optimal value of the beam convergence angle which is mainly restricted by the spherical aberration effects and the thermal transverse velocity effect of electrons in the source. From Figure 2-5 it can be inferred that a system with $\mathrm{C}_{\mathrm{C}}=10 \mathrm{CM}, \mathrm{C}_{\mathrm{S}}=40 \mathrm{CM}$, spread energy of the beam $=2 \mathrm{~V}$, source current $=1 \mathrm{E}-8 \mathrm{~A}$ and accelerating voltage $=20 \mathrm{KV}$ a minimal radius of $100 \mathrm{~nm}$ is obtained. However, research has made a lot of efforts to improve aberration and thermal transverse 
velocity effect that beams with disk of least confusion as small as $5 \mathrm{~nm}$ has been reported. ${ }^{35}$ This fact has made transmission scanning electron microscopy (TSEM) success.

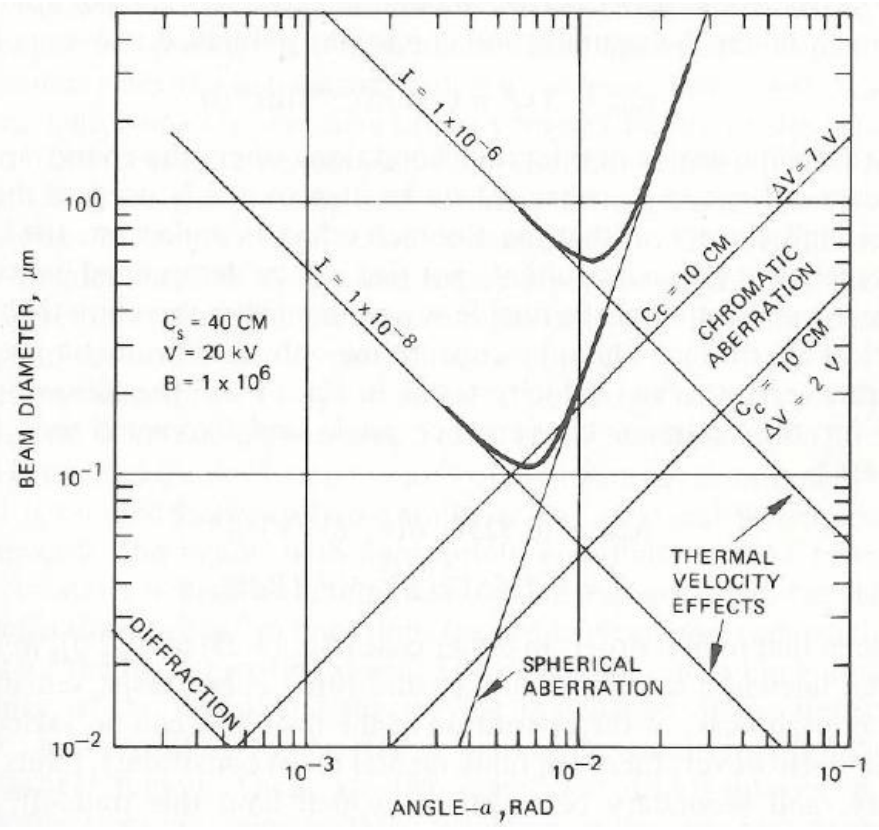

Figure 2-5: Aberrations, diffraction and thermal transverse velocity effects. 34

Like in any other optical systems, in this type of columns astigmatism effects are present. This can result from imperfection on the circular openings in the apertures or on the openings on the magnetic lenses. Any tilting or displacements of these elements can cause astigmatism, as well. These effects can misshape the cross section of the beam, rather than circular this will make the beam elliptical, this is also another kind of aberration and it is called ellipticity astigmatism. This astigmatism, mainly induced by mechanical properties of the elements, can be corrected by coils around the column called stigmators; these stigmators are constructed by quadrupole and placed around the column to shape up the beam by means of transverse magnetic fields. In this way an incoming elliptical beam can become a perfectly round-shape beam. Figure 2-6 illustrates a quadrupole arrangement for astigmatism correction. For better control with astigmatism correction, octupole configurations have been designed to correct higher and lower order of astigmatism with independently pole orientation. ${ }^{36}$ 


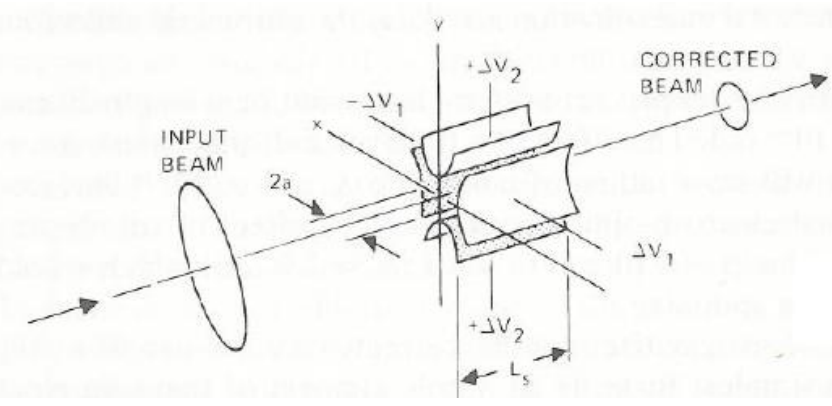

Figure 2-6: Quadrupole stigmators, beam cross-section. 34

\subsubsection{Electron interactions}

When a specimen is hit with a beam of electrons, the atomic structure and the high energy electrons interact with each other generating various types of signals that allow an SEM system to be able not only for imaging but also for chemical analyzing (specimen composition). These signals include secondary electrons, Auger electrons, back scattered electrons, x rays, cathodoluminescence among others, Figure 2-7 displays all these different signals and its depths in the specimen.
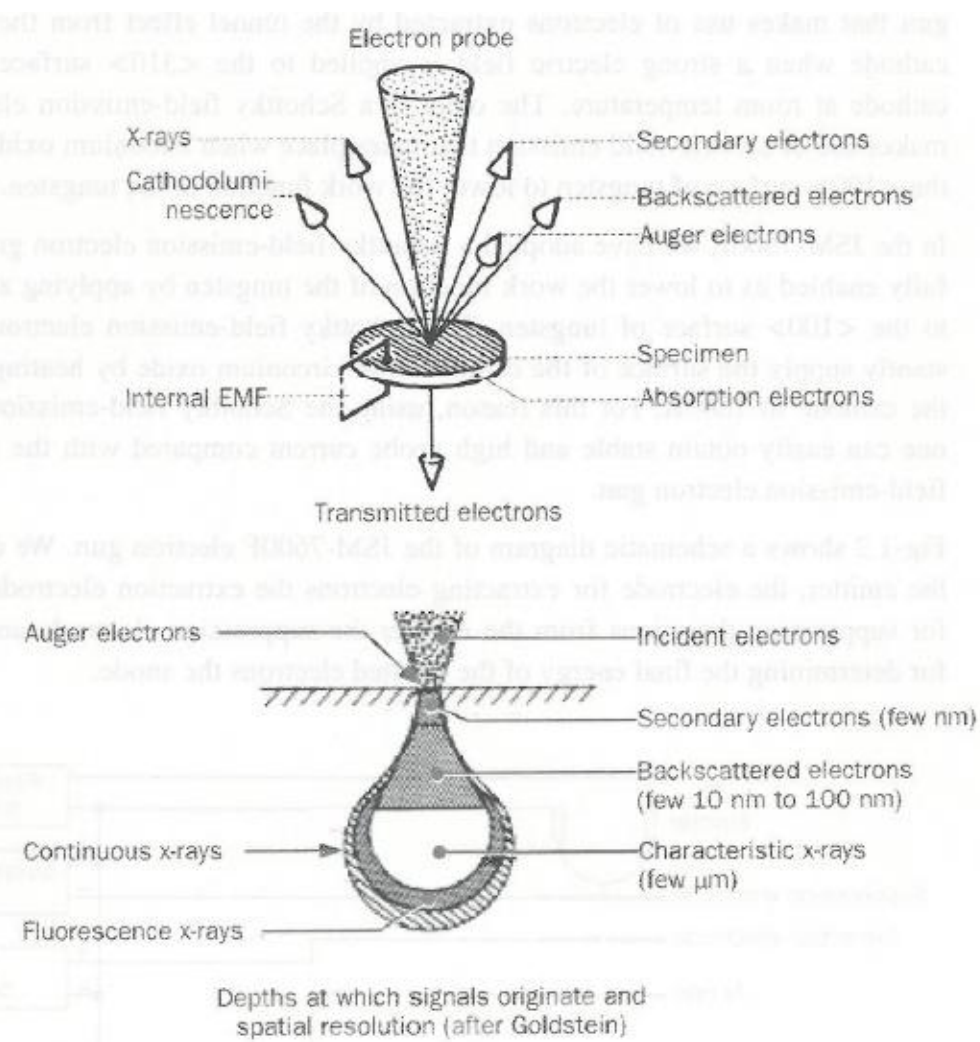

Figure 2-7: Different signals generated by the specimen upon electron radiation. 32 
For EBL penetration depth in the substrate of high energy electrons and secondary electrons are the main concern on the process. In general, in EBL the specimen will be coated with an electron sensitive resist on top, this film and the substrate itself will provoke scattering events to the electrons coming from the beam which causes undesired exposure in areas that should not be exposed (shape distortion of the original pattern). Even though, EBL is a high resolution writing method, it is limited by aberration on the lenses but most important by scattering of electron in the resist and the substrate. This is called proximity effect and it depends on many factors like specimen atomic number, substrate and resist thickness, density and geometry of the pattern to be written. This electron scattering splits into two types: forward scatter and back scatter. In the former, electrons collide with electrons in the resist or the substrate, this causes a small deviation on the incident electron and part of the electron energy is given to the atom. This type of collision is called inelastic and is characterized by small angles of deflection. The latter type of scattering has to do with the collision of high energy electrons with solid heavier nucleus of atoms in the structure, which produces elastic collisions. This results in conservation of electron energy and changes in the electron trajectory. The scattering angle in this case is larger. Because of these large scattering angles, high energy electron may come back up to the resist at small distances from the incident beam and expose undesired areas; this scattering is what provokes the so called proximity effect.

While primary electrons travel through the resist, they give up part of its energy as secondary electrons. This low energy electrons are responsible for the exposure of the resist, their energy ranges from 2 to $50 \mathrm{eV}$ so they can move few nanometers before they lose all its energy, thus, they contribute very little to the proximity effect but they still affect the size of the features in the pattern. It is important to mention that the energy of electrons cannot be higher than the displacement energy of the substrate; otherwise, damages can be caused to the crystal structure of the substrate.

To better understand this scattering of electrons and proximity effect, many Monte Carlo (MC) simulations have been performed to evaluate how these kind of scattering affects the trajectory of primary and secondary electrons in the resist and substrate. ${ }^{37}$ Figure 2-8 displays some MC simulations for analyzing primary and secondary electron scattering on PMMA (positive tone resist) and on Si substrate for different accelerating voltages. 


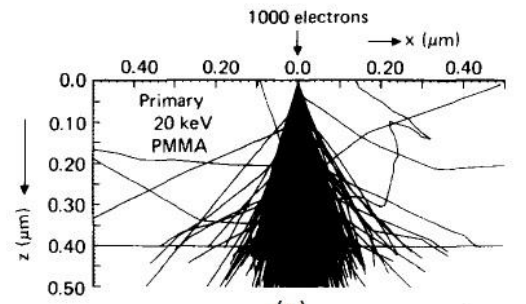

(a)

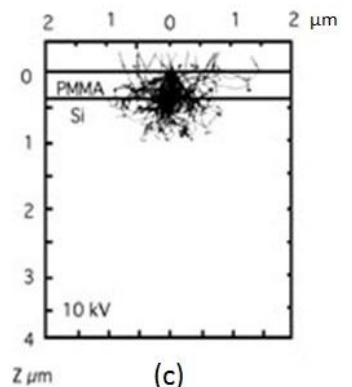

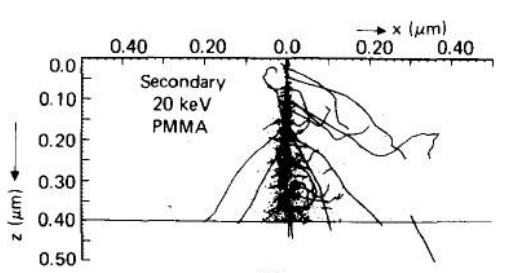

(b)

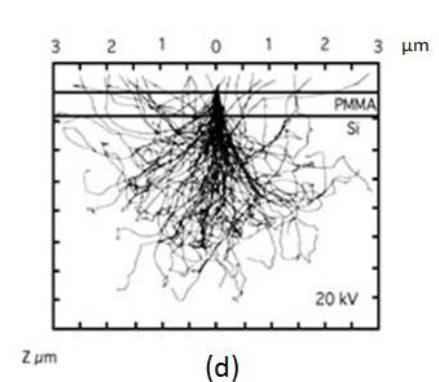

Figure 2-8: Electron scattering: (a) Forward scattering of primary electrons, (b) Forward scattering of secondary electrons, 37 (c) and (d) Forward and backscattering of electrons in resist and substrate as function of accelerating voltage. 33

In order to correct this proximity effect many algorithms have been implemented and have required a precise knowledge about the energy density been deposited in the resist film. This energy density profile is approximated as a double Gaussian distribution and it is function of the setup process. ${ }^{38}$

$$
f(x)=\frac{1}{1+\eta}\left(\frac{1}{\pi * \alpha^{2}} e^{\frac{-r^{2}}{\alpha^{2}}}+\frac{\eta}{\pi * \beta^{2}} e^{\frac{-r^{2}}{\beta^{2}}}\right)
$$

Where:

$\eta$.- ratio between backscattered energy to forward scattered energy

$\alpha$.- forward scattering range parameter.

$\beta$.- back scattering range parameter.

These parameters are called proximity parameters and they are function of the resist, substrate and the accelerating voltage on the system. Usually, these parameters are obtained experimentally or by means of MC simulations. This double Gaussian distribution representing the scattered beam has been an accurate approximation; however, it is been found that this is not enough for some specific types of substrates, substrate with multiple films on top or for sub-100 nm scale. Therefore, functions more complex has been implemented including a third Gaussian distribution or multiple Gaussian distributions. ${ }^{39}$ All these models simulate $2 \mathrm{D}$ energy distribution when in reality this is an issue that deals with 3D, having as an important parameter the depth. Although, there are some reason why it is studied as a $2 \mathrm{D}$ problem and they are: simplification of simulation time and the difference between profiles for 
different depths are in the range from 0 to $10 \mathrm{~nm}$ which is way smaller than the features to be written in the resist, then a $2 \mathrm{D}$ simulation results in quite accurate approximations. In order to implement a correction to the proximity effects some algorithms have been studied, so that high feature density patterns and complicated feature geometries in a pattern can be written. These schemes for proximity correction include: 40

Dose modulation - This method determines the dose for each point of exposure which will be accurate enough and moderately ease to be computed. So that big features receive less exposure times and small features receive larger doses.

Shape modification - This method uses single exposure dose for the whole pattern writing, but some specific shapes of the pattern will be modified, thus the developed pattern will look pretty much like the original design. This scheme make use of tables to adjust the dose for each rectangular circuit element, also each edge facing other elements will be adjusted. PYRAMID is software that has been designed to perform these duties to correct the proximity effect when performing e-beam writing. ${ }^{41}$

Background exposure correction - This method does not use any computation to correct the proximity effect instead it writes a second exposure that is inversed of the original one. This technique is known as GHOST. Basically what it does is to apply a uniform background dose. This method only takes care of backscattering, so forward scattering is still going on. The fact that the pattern should be written twice decreases the throughput of the process yet it is easier to use because no computations are needed. ${ }^{33,42}$

Now that all these issues have been understood, it is necessary to clarify that our system has NPGS software for EBL, which does not have any tools for correction of proximity effect. By 2008, a new software hardware system called Nanomaker was released; this new packet was able to do EBL on SEM based systems but the novel thing was that this system has tools for proximity effect correction. Later on 2009 Nanomaker reported the possibility to export proximity parameters to NPGS software for writing. This software has successfully demonstrated capability of writing of $25 \mathrm{~nm}$ width lines separated $50 \mathrm{~nm}$ from each other. Hopefully this could be implemented in our system so better feature resolution can be obtained for future PhCs fabrication.

\subsubsection{Resist}

Electron sensitive resist is the mean where the entire pattern by EBL is recorded. This resist is usually a liquid polymer that will be dissolved later in solvent in a process called developing. Resist is spun onto the substrate and later hot plate baked to evaporate any kind of solvent left in the resist. EBL 
will change the internal structure in the resist and depending on the tone of the resist, this will become more solvable (positive tone PMMA) or less solvable (ma-N 24xx) when developing. And after developing processes, final pattern is treated one more time if this is going to work directly as a hard mask for etching processes, otherwise, the pattern is taken directly for physical deposition for lift off processes. $^{33}$

Positive resist - upon exposure to the beam, electrons, traveling through the resist, break polymer molecular chains, causing fragments of lower molecular weight, so when developing this exposed film, all the areas with low molecular weight are removed

Negative resist - upon exposure to the beam, the resist molecular chains star cross-linking together, and then the resist becomes less solvable in developer solutions.

All type of resists, positive or negative tones, look for higher resolution as a very important parameter. This parameter defines how steep the sidewalls in the features of the pattern are. Due to throughput of this process high speed for developing is required but that decreases resolution in the pattern, the reason is because high resolution resists are less sensitive, therefore, they take longer when developing. In EBL to reduce the proximity effect and get accurate feature sizes, the exposure to the positive resist should be low enough so washing away of the undesired resist is still possible, leaving behind the original pattern. Also to diminish proximity effect the thickness of the resist is reduced to the minimum so forward scattering is reduced but the resist should be still thick enough so it is able to undergo etching or lift-off processes. For negative resist, higher aspect ratios are achievable increasing the accelerating voltage in the column of the SEM, but it should be noticed that this would increase backscattering effects because of high energy electrons.

As it was said, the resist that has been used for this work is a liquid polymer, charging effects on the resist can be produced when the substrate is not conductive or even when it is a semiconductor during exposure. Charge effect in EBL means that electrons start locating themselves on one location what causes the deflection of the electron beam when this scans close to this location (particles with the same charge repel each other) producing terrible distortions on the pattern when imaging or writing. To relief this effect for EBL, a thin metal film is deposited underneath the resist. This thin metal is often Au or $\mathrm{Au} / \mathrm{Pd}, \mathrm{Cr}$ or even $\mathrm{Ni}$ which have good conductivity quality. Table 2-1 summarizes the main properties for the electron beam resists that have been used in this work. 


\begin{tabular}{|c|c|c|c|c|c|}
\hline & Tone & Resolution [nm] & $\begin{array}{l}\text { Sensitivity at } \\
20 \mathrm{kV}\left[\mu \mathrm{C} / \mathrm{cm}^{2}\right]\end{array}$ & Developer & Removal \\
\hline PMMA 496 & Positive & 10 & 100 & MIBK:IPA & Remover PG \\
\hline ma-N2403 & Negative & 50 & 80 & ma-D 525 & $\begin{array}{l}\text { mr-REM } 660 \text { / } \\
\text { Remover PG }\end{array}$ \\
\hline
\end{tabular}

Table 2-2: Main properties for PMMA and ma-N resists.

\subsection{PHYSICAL VAPOR DEPOSITION}

In micro fabrication there has always been the necessity to go from semiconductor to metals for examples metal contacts, metal interconnects, metal wires and also hard metal mask for pattern transfer. Physical vapor deposition is a coating technique that relates the transfer of vaporized material coming from a solid or liquid source onto a substrate. This process takes place under high vacuum conditions and starts when evaporating the source through a high energy e-beam (e-beam evaporation) or ion (sputtering) bombardment, the energy transmitted to the material causes to the atoms to be ejected away producing their evaporation. These atoms have enough energy to move through the chamber at vacuum until they locate themselves onto the substrate building up a coating layer.

Often, not only metals need to be deposited but also compounds like: oxides, nitrides or carbides; hence evaporated atoms, from the source, are forced to react with ions coming from a different gas source before they are placed onto the substrate. These ions can come from $\mathrm{N}_{2}, \mathrm{O}_{2}, \mathrm{CH}_{4}$ gases to get the compounds before mentioned. This process is called ion beam assisted deposition.

\subsubsection{Electron-beam evaporation}

This technique takes advantage of the fact that for a determined material temperature there is an equilibrium vapor pressure above the material. Matter states can go from solid to liquid or gas state when applying heat to it; if the material temperature is under the melting point the process is called sublimation and if it is above is called evaporation. In this physical deposition method, material is heat up till this becomes molten because this state renders a region with high vapor pressures which means high relative deposition rates. Graphite crucible liners are used to contain the melted material to avoid contamination. ${ }^{43}$

To heat the material, this evaporation method relies on the transfer of high kinetic energy of electrons to thermal energy which is strong enough to melt and vaporize the material source. Electrons are extracted from a tungsten filament and accelerated into the material. This micro electron beam creates a spot of heat in the material; as a result a very narrow atom beam is obtained from the source. In order to increase the area of this atom beam and heat the entire crucible area uniformly, the micron electron beam 
is swept in circles providing a uniform area of heat to the source. ${ }^{44}$ A magnetic field is used to bend the micro electron beam $270^{\circ}$ into the source, so a shield can be provided to the tungsten filament to avoid contamination. An e-beam evaporator schematic is shown in Figure 2-9.

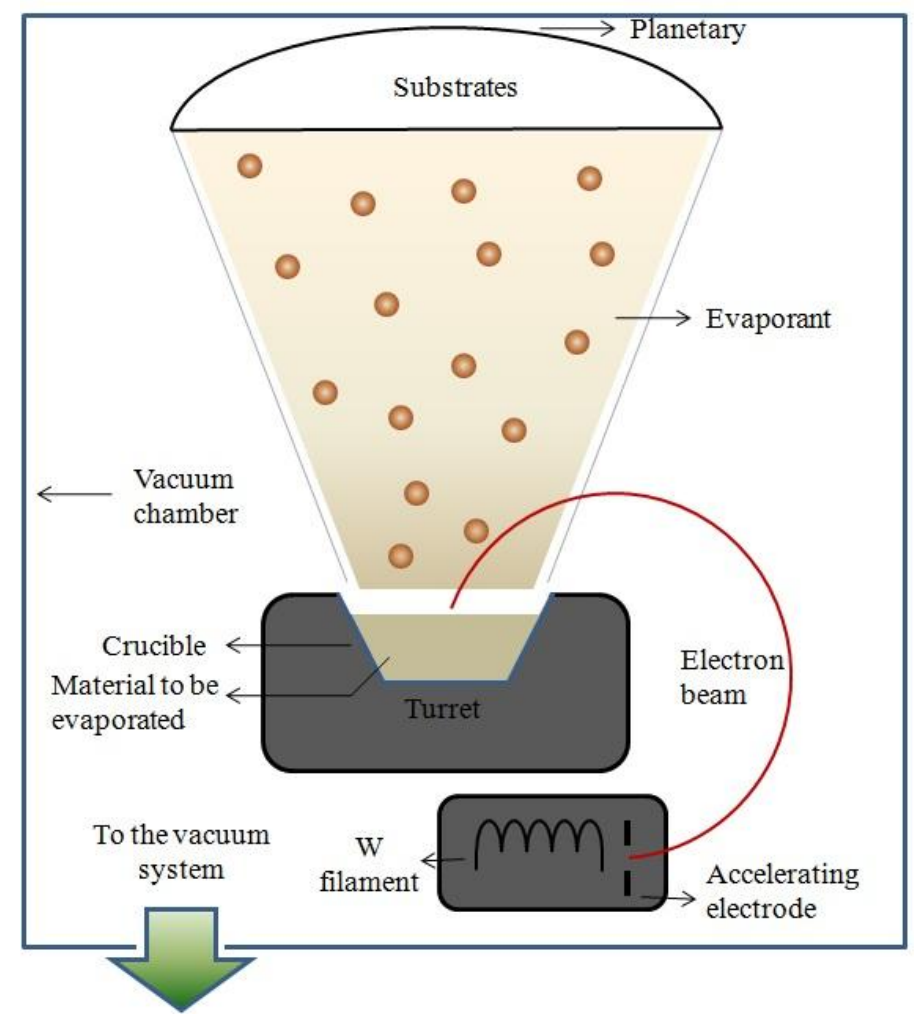

Figure 2-9: Electron Beam Evaporator schematic.

A very uniform coating layer is achieved if the wafer holder is constructed with a spherical-like shape commonly called planetary. The deposition rate of the material being deposited onto the substrate is measured with a quartz crystal rate monitor. This sensor measures resonance frequency and while the material is being deposited on top of this sensor, the resonance frequency is shifted what translates into material thickness.

E-beam evaporation is well known for its high purity deposition; however, step coverage with these systems is really poor due to directional nature and long free paths of atoms inside the chamber. Shadow effects and discontinuities of the film are present when coating over a step. To solve this issue, the planetary is rotated to eliminate the shadow effect. Also, the sample is heat up to improve the atom diffusion over the substrate improving coverage but still discontinuous films are obtained for steps with aspect ratios greater than 1. Figure 2-10 shows the shadow effect in a step coated with and without sample rotation and heat. 


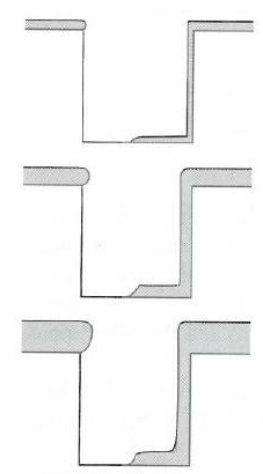

(a)

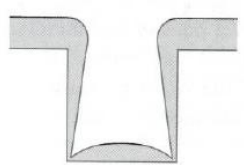

(b)

Figure 2-10: Shadow effect on a step in a sample, (a) neither rotated nor heated, (b) rotated and heated. ${ }^{43}$

Coating discontinuities on high aspect ratio features makes e-beam evaporation helpless for metallization but great for pattern transfer using lift-off technique. These discontinuities on the steps give way to any solvent to attack and remove the resist underneath the film washing away unwanted metal parts.

\subsubsection{Ion beam assisted deposition (IBAD)}

This method, used for compound deposition, is set up inside the same electron beam evaporator. An ion gun is placed at the bottom of the chamber at an angle of $60^{\circ}$ and pointing towards the sample. This gun can inject an energetic broad beam of ions (any gas previously mentioned) simultaneously with the evaporation of materials, so they can react with each other habilitating the system to deposit compounds onto the substrate. Ion-assisted evaporation is mainly used to deposit oxide composition like $\mathrm{SiO}_{2}, \mathrm{Al}_{2} \mathrm{O}_{3}$, $\mathrm{TiO}_{2}$, and $\mathrm{Ta}_{2} \mathrm{O}_{5}$, nitrides like $\mathrm{Si}_{3} \mathrm{~N}_{4}$ and carbides, as well. The ion source consist on an anode and a cathode separated with a gap where a gas runs through it, a high electric field is applied to ionize the gas creating plasma and starting ion bombardment. These guns are end-Hall type and the ion beam is neutralized with an independent electron source (a tungsten filament) to avoid any charging from the ions and keep the plasma neutral. An IBAD scheme is illustrated in Figure 2-11. 


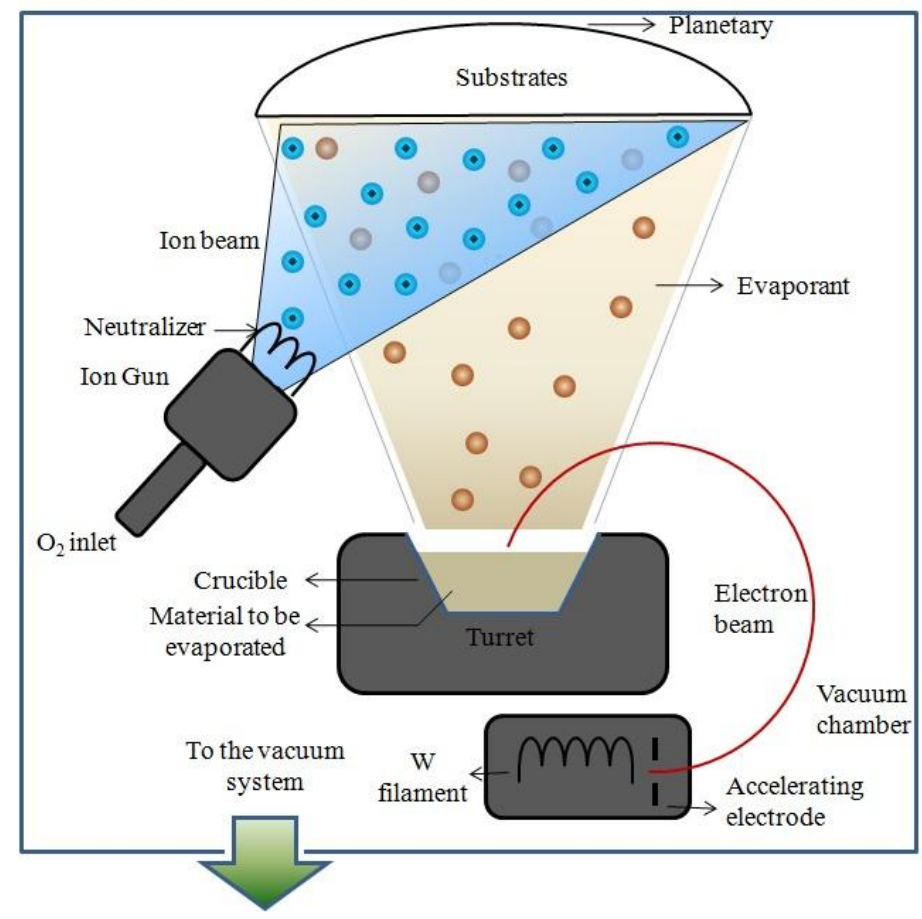

Figure 2-11: Schematic of Ion assisted beam deposition system.

\subsection{ETCHING TECHNIQUES}

Usually, etching process is related to subtractive techniques for pattern transfer in micro fabrication, and involves material removal from the substrate. This step takes place in micro fabrication after a mask has been created on a substrate and unwanted material needs to be taken away. Depending on the type of etching, this could remove material in the horizontal or vertical direction. Anisotropy is a term that measures the rate between horizontal and vertical etching rates and the process is named completely anisotropic when this ratio equals unity. Etching removing can be performed physically or chemically or a combination of both. Figure 2-12 shows the different mechanisms of etching based on the environment pressure where process is executed.

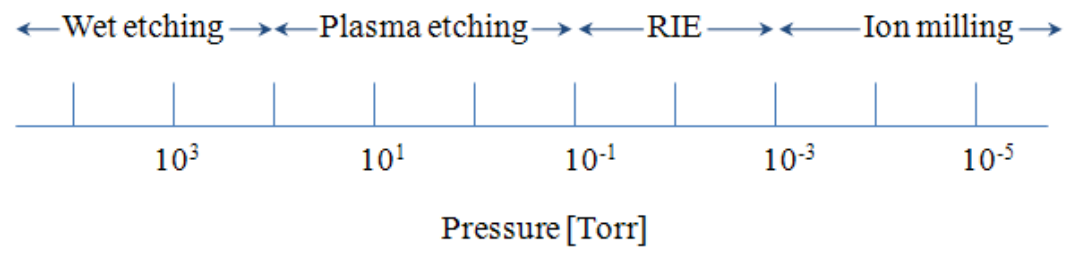

Figure 2-12: Types of etching mechanisms depending on environment pressure. ${ }^{43}$ 


\subsubsection{Wet etching}

On the early ages of micro fabrication this used to be the most popular method for removing material, later on, as feature sizes started shrinking lots of problems emerged with this technique. This etching method is strictly chemical and it can be divided in two classes, isotropic and anisotropic.

Isotropic wet etching is characterized by the undercut just underneath the mask due to removing of materials in all the direction (vertical and horizontal). Some advantages and disadvantages are described next:

- Lack of anisotropy makes impossible to fabricate small features.

- Poor depth control, since the etchants are liquids there is no way to stop the etching process precisely.

- Any imperfection on the mask due to under developing or overexposure can prevent from wet etching the substrate.

- Waste a lot etchant after use, which make this technique expensive

- High selectivity and the fact that this process does not produce any surface damage.

- Does not require any dedicated equipment but only appropriate hoods.

Despite all the disadvantages, wet etching has been used for Si micromachining. For nanofabrication new techniques needed to be implemented. Wet etching is mainly used for cleaning substrates due to the high selectivity for removing any kind of contamination particles. Figure 2-13 illustrates a cross section of a typical wet etching process.

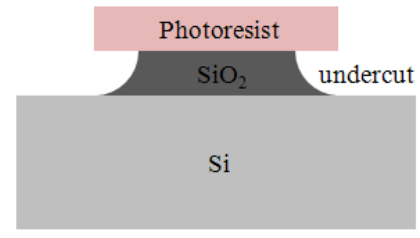

Figure 2-13: Isotropic wet etch process. ${ }^{45}$

When performing wet etching, agitation can produce a uniform transport of etchants in cavities what induces the same etching rate along all the directions in the substrate. Figure 2-14 depicts this agitation effect on wet etching processes.
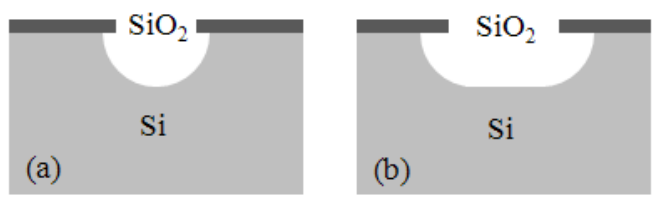

Figure 2-14: Isotropic wet etching: (a) with agitation, (b) no agitation. ${ }^{45}$ 
Anisotropic wet etching is also possible but extremely depending on the crystal structure of the substrate. This advantage is widely used in $\mathrm{Si}$ micromachining to etch very deep trenches or in IC microfabrication to isolate devices from each other. This process is based on specific etchant that remove material in a way faster along certain crystal directions of the Si substrate. This directional etching is a strong function of the density of bonds between atoms on the different direction along the crystal structure, hence, the wet etching will be faster on the direction with the least number of bonds, as a consequence of this peculiar etch, sharp facets with very accurate angles are obtained. Figure 2-15 shows this kind of wet etching and the different crystal directions involved in the process.
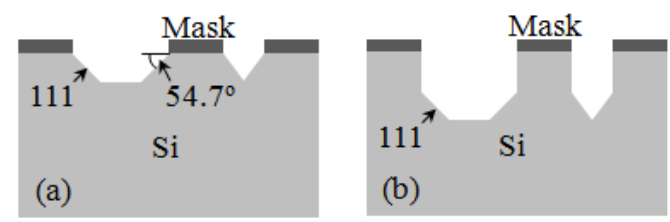

Figure 2-15: Cross section of anisotropic wet etching in: (a) (100) orientation; (b) (110) orientation. ${ }^{45}$

\subsubsection{Dry etching}

Etching as important as it is in micro fabrication needed to overcome the issues presented with wet etching approach; therefore, many new etchants, especially dry types, have emerged. Also, new etching systems have been implemented to satisfy the urgent necessity of etching new geometries and new materials (metal, silicides, semiconductors, dielectrics) in micro fabrication.

Plasma etching solved most of the serious problems related with wet etching processes; however, it was not able to keep uniformity on the etching process even from one wafer to another. Many other systems, derived from this principle, were created to improve uniformity, anisotropy, process control and complete automated processing. This plasma based etching can be classified in the following: chemical etch, physical etch, reactive ion etching (RIE), and deep reactive ion etching (DRIE). The last two categories have been the best options adopted in microfabrication. RIE is a method that combines chemical and physical bombardment simultaneously, plasma is the source that provides with chemical etchant agents and energetic ions for the dry etch process. In these systems, etch is a chemical reaction and its reaction rate depends on the ionic bombardment. RIE is characterized by its high anisotropy when etching. Thought, this is coupled with the rate reaction, which means that there is a tradeoff between anisotropy and reaction rate on the process. This effect has to do with the fact that for higher reaction 
rates, a higher concentration of reactive species are required which in turn means a increase in pressure what causes an increase in the probably of particle collisions which finally sacrifice anisotropy.

Reactive ion beam etching (RIBE), magnetron ion etching (MIE), Inductively couple plasma RIE, electron cyclotron resonance plasma etching (ECR) are systems with the capacity of high density plasma generation what represents a good option to overcome such limitation previously discussed. Nevertheless, these systems are plasma based, what presents some drawbacks like high temperature operation and faster erosion of the resist, but that is the trade-off to get better structure resolution in micro fabrication. ${ }^{46}$

\subsubsection{Inductively coupled plasma reactive ion etching (ICP-RIE)}

Plasma is a process that involves matter on the fourth state; this matter can be either charged ions or neutral atoms and radicals that reach an equilibrium status. This etching technique is based on the momentum transfer between energetic particles and the atoms in the substrate. If the plasma has high energetic ions (Ar) they can hit the substrate and knock off atoms from it, this is called physical etching or sputtering etching. This approach cannot differentiate between material chemistry, so that is why this technique has very poor selectivity but high anisotropy. To improve selectivity, plasma is based on atoms and radicals that can chemically react with the atoms in the substrate forming volatile compound that are easily pumped away. These systems are capable of letting the user to vary the amount of physical etching (ion energy) and chemical (gases inlet) etching. When equilibrium between these two processes is reached, the etching process becomes highly anisotropic. The etching rates were relatively low due to plasma density, because the only way to increase the density of species in the plasma was to increase the RIE power (ion energy). Inductively coupled plasma is an implementation added to RIE systems, which let plasma density be uncoupled from RIE power, creating very high density plasmas at low vacuum pressures. The ICP-RIE architecture is shown in Figure 2-16. 


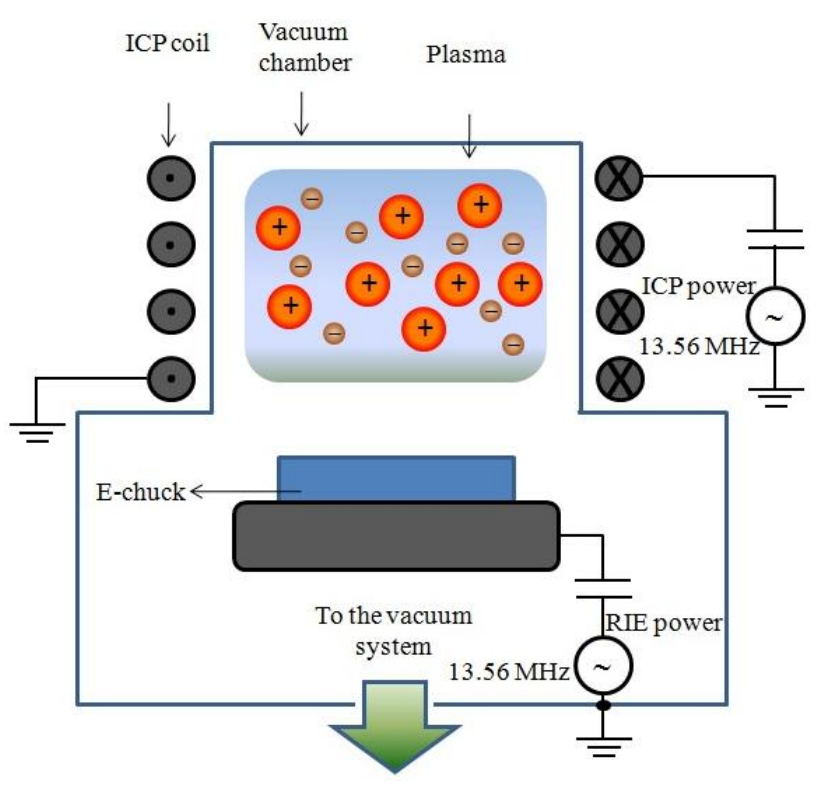

Figure 2-16: ICP RIE schematic.

This system has a chamber under vacuum, where the plasma takes place. This chamber is enclosed by an external water-cooled coil (ICP coil) where RF power (ICP power) circulates at 13.56 $\mathrm{MHz}$, this is the primary plasma source. The chamber also houses an E-chuck that is able to generate a negative DC bias to increase ion energy in the plasma to get high anisotropy as etching is performed. This E-chuck possesses also the ability to generate a strong electric field to clamp the sample holder down and let Helium to cool down the back of the sample. RIE and ICP power generator have matching networks to keep approximately constant ion energy and density plasma during the process. This etching process starts when the chamber with a background vacuum pressure is filled uniformly with gases $\left(\mathrm{CF}_{4}, \mathrm{O}_{2}, \mathrm{~N}_{2}\right.$, $\mathrm{Cl}_{2}, \mathrm{BCl}_{3}, \mathrm{Ar}$ ) accurately measured, then ICP and RIE power generators are turned on, this causes that the gas inside the chamber ionizes and plasma ignites. ICP power confines the plasma towards the center of the chuck (high density plasma) and RIE power accelerates the ions to the sample keeping the pressure at low levels. As a consequence, etch rate and anisotropy are improved, simultaneously. ${ }^{45}$

In order to accomplish high aspect ratios (highly anisotropic etching) with this high density low pressure systems the following aspect are the main concern: decreasing the reaction on the walls and reducing the lateral etch and implementing a sidewall passivation protection from radical, atomic and neutral etchant species by means of polymerizing chemistries. ICP RIE etching has demonstrated vertical sidewall profiles by simultaneously performing etching and sidewall passivation processes by means of mixing the right precursors gases depending on the type of material to be etched. To explain the chemistry and physics of this system, a $\mathrm{Si}$ etching process in $\mathrm{SF}_{6} / \mathrm{O}_{2}$ plasma can be described as follows:

Electron impact dissociation generates radicals and ions in the plasma: ${ }^{45}$ 


$$
\begin{gathered}
S F_{6}+e^{-} \rightarrow S_{X} F_{Y}^{+}+S_{X} F_{Y}^{*}+F^{*}+e^{-} \\
O_{2}+e^{-} \rightarrow O^{+}+O^{-}+e^{-}
\end{gathered}
$$

Oxygen ions react with the Silicon forming $\mathrm{SiO}^{2}$ which becomes the sidewall passivation:

$$
O^{*}+\operatorname{Si}(\text { surface }) \rightarrow \operatorname{SiO}_{n}(\text { surface film })
$$

Then the bottom is also covered with this film which needs to be etched away anisotropically, so Si film can be etched next by the Fluorine ions:

$$
\begin{aligned}
\mathrm{SiO}_{n}(\text { surface film })+\mathrm{F}^{*} & \rightarrow \mathrm{SiO}_{n}(\text { surface film })+F \\
\text { Ion energy } & \\
\mathrm{SiO}_{n}(\text { surface film })+F & \rightarrow \operatorname{SiF}_{x}(\text { ads })+\operatorname{SiO}_{x} F_{y}(\text { ads })
\end{aligned}
$$

Where ads stands for adsorption of Fluorine ions onto the Si surface. These ions help the adsorption, reaction and desorption processes. The bare silicon now can be etched by Fluorine ions and product formation desorption takes place as gas to be pumped away.

$$
\begin{gathered}
S i+F^{*}->S i+n F \\
\text { Ion energy } \\
S i+n F \rightarrow S i F_{x}(\text { ads }) \\
S i F_{x}(\text { ads }) \rightarrow S i F_{x} \text { (gas) }
\end{gathered}
$$

It can be claimed that ion flux enhance anisotropy, and the removal of the passivation layer and the immediate etching of silicon should be balanced, therefore a vertical profile on the sidewalls can be achieved. Figure 2-17 (a) shows the sidewall along with the passivation film. A summary of the major steps in RIE is presented in Figure 2-17 (b) these include: generation of reactive agents (ion or radicals) like Fluorine $\mathrm{F}^{*}$ in our case, diffusion, adsorption, and reaction at surface, desorption of reactive clusters and finally diffusion of by products. 45

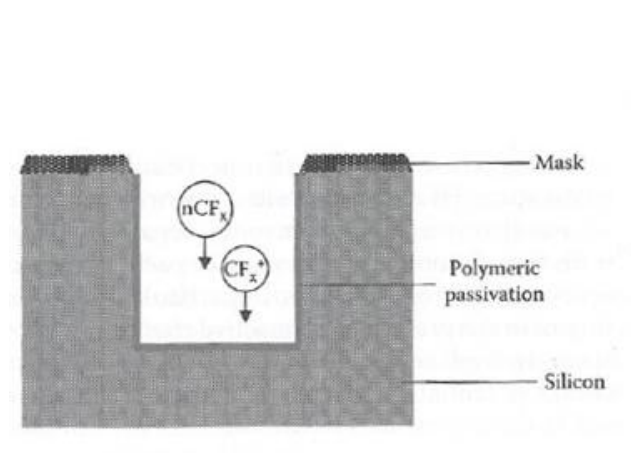

(a)

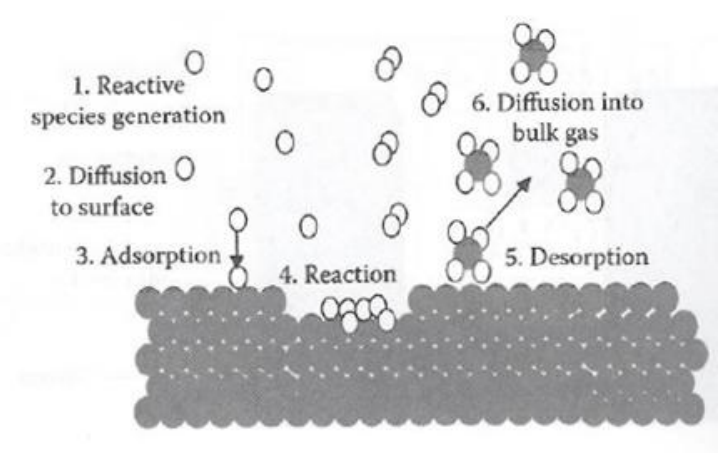

(b)

Figure 2-17: (a) Etching process and passivation sidewalls; (b) Scheme of RIE process. ${ }^{45}$ 
ICP-RIE system can perform high aspect ratio etching with high anisotropy in microfabrication, however, etch rate, etch selectivity, and surface morphology demonstrate a high dependence on the system variables. These variables can be named macroparameters and they include: gas flow rate, ICP and RIE radiofrequency powers, chamber pressure and wafer temperature. All these macroparemeters need to be monitored and controlled to optimize the etching process according to the microstructure needs. For example if a dicing line is needed, surface morphology is not important; instead, high etching rates are required. On the other hand, smooth and anisotropic sidewalls are extremely important in photonic crystal fabrication rather than high etch rates. For this reason, an understanding of the influence of every single macroparameter is vital to maximize the overall efficiency of RIE ICP systems.

RIE power determines the acceleration of ions to a high enough energy to bombard the passivation layer on the bottom as keeping the sidewalls undamaged, thus a vertical profile is achieved. Then, etching rates, etch selectivity and etch sidewalls are strong functions of RIE power. Of course, if the energy of ions is higher etch rate increases and also the erosion of the mask speeds up, decreasing the selectivity of the mask to the material, undercut effect is the main problem when RIE power is too low.

ICP power is also related with etch rate. An increase on this parameter can produce an increase in the number of reactive species and at the same time confine them in the center of the camber. It is important to mention that the implementation of this parameter let the system possess high density plasma that is not function of RIE power; therefore, an increase on this parameter means a increase in the number of reactive etchant species per unit volume independently of the energy that the particles have and the low chamber pressure.

Gas flow into the chamber is another parameter that also is related with the etching rate. In this case, if gas flow is poor, the limitation on etch rate would be due to insufficient amount of reactive etch compounds, nevertheless an excessive increase in gas flow does not mean an infinite increase in etch rate, and this is because of kinetic aspects in formation and loss of the reactive compounds.

Chamber pressure is a macroparameter that deals with the number of collision of particle in the plasma. This parameter affect etch rate, etch selectivity and sidewall profiles. When pressure increases, the gas increases inside the chamber what causes the increase of the etching rate, even though, as pressure increases more and more etching rate stops because dissociation efficiency in the plasma is too low and the reactive compounds decrease. If pressure keeps going up, the number of collision between particles increase due to a reduction in free path distances what causes a higher recombination between reactive ions decreasing the etching rate. Also, due to the increase of collisions, the etching becomes isotropic ruining the verticality of the etching profiles. Bowing effect is a problem that is noticeable when chamber pressure is too high. Consequently, low pressure levels are required for vertical profiles but this decreases 
the selectivity between the material to be etched and the mask; low pressures increase the mean free path of particles what causes an increase of the etching rate of the mask. ${ }^{45,47}$ Figure 2-18 (a) shows most of the etching effects that have been discussed about macroparameters variations.

Plasma based etching process depends not only on the macroparameter that were explained previously but on the plasma itself. Loading effect is a problem that is caused by non uniform plasma distribution, non vertical profile of hard masks and the density of features. This effect can be divided into two levels: microloading and macroloading. The former, is a consequence of variations on the areas of the substrate being exposed to the plasma; this produces deeper etching depths on larger features and it is caused by the local depletion of etchant in features with high aspect ratios. And the latter has to do with the global variations of substrate being exposed to the plasma. Thus the etching rate depends on pattern distribution and the overall substrate areas being exposed. Figure 2-18 (a) shows a summary of effects of non optimized macroparameters on high density plasma etching; (b) shows the loading effect on features with different areas and its depths. ${ }^{45}$

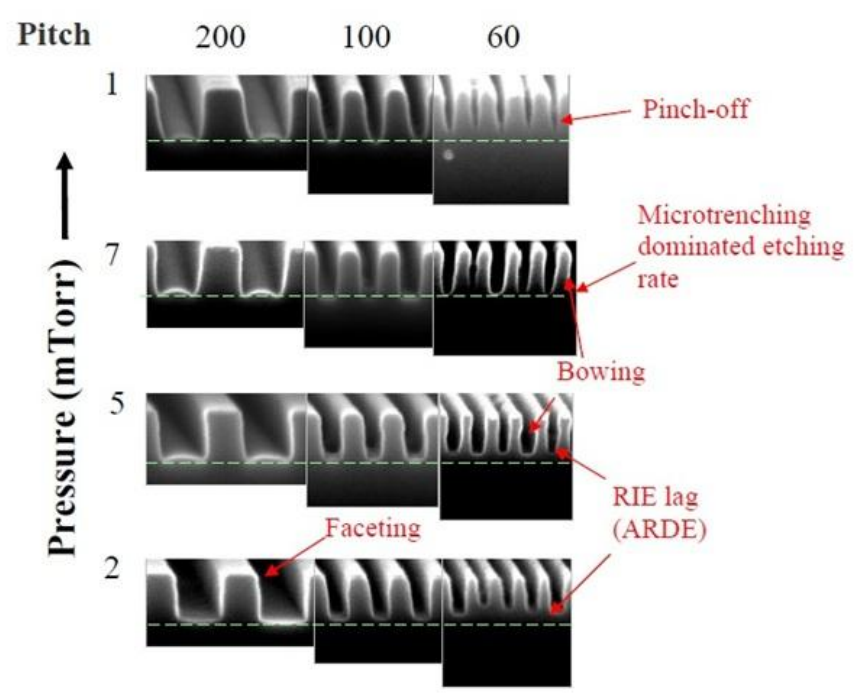

(a)

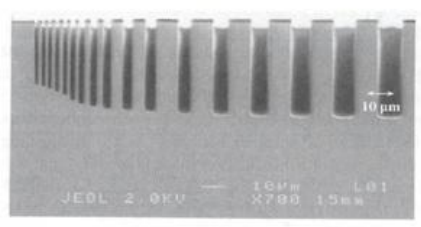

(b)

Figure 2-18: (a) Etching effect due to non optimized macroparameter, ${ }^{48}$ (b) Loading effect. ${ }^{45}$ 


\section{CHAPTER III}

\section{PHOTONIC CRYSTAL FABRICATION}

\subsection{INTRODUCTION}

During typical top-down nanofabrication computer-designed CAD file that contains nanostructural patterns is reproduced onto a resist using an EBL system. The resist image is then transferred onto the substrate using additive or subtractive nanofabrication methods. The main goal of this chapter is to optimize the process that allows the successful fabrication of PhC structures on $\mathrm{Si}$, GaN and ITO for operation at IR and visible wavelengths. To achieve the goal, the optimal recipes for two critical fabrication steps, e-beam lithography and dry etching, must be developed.

\subsection{OPTIMIZATION OF E-BEAM LITHOGRAPHY}

The process of EBL is important in PhC fabrication because the resolution of features in the final material will depend on the quality of the resist mask defined by EBL. The optimization is needed to figure out the right amount of dose exposure and beam energy in order to successfully transfer the $\mathrm{PhC}$ patterns to the resist. In this work, we optimized the lithography recipes for both negative and positive resists on different substrates, including $\mathrm{Si}, \mathrm{GaN}$ and ITO.

Commercial $\mathrm{Si}$ and GaN wafers were used in this study, whereas ITO was deposited using e-beam evaporation deposition on $\mathrm{Si}$ substrates. Before the lithography step, all the wafers were cleaned to remove surface contaminants and native oxides. This also enhanced the adhesion of resist to the wafer surfaces. In this work Si cleaning procedures are described below.

1. Silicon wafers were cleaned with acetone (ultrasonic bath), methanol, and DI water for 5 min each for removing any organic impurities, and then etched in $\mathrm{HCl}: \mathrm{HNO}_{3}(3: 1)$ and buffered oxide-etch solution in order to remove any metal and oxide impurities. As a final cleaning step, wafers were submerged in fresh running De-Ionized (DI) water for $5 \mathrm{~min}$. Subsequently, wafer surfaces were dehydrated on a hot plate at $120^{\circ} \mathrm{C}$ for $10 \mathrm{~min}$.

2. GaN wafers were cleaned with acetone (ultrasonic bath), methanol, and DI water for 5 min each for removing any organic impurities. In order to remove any metal and oxide impurities, the samples were etched in $\mathrm{HCl}$ and next in Buffer Oxide Etchant (BOE) for 1 min and 2 min respectively. Finally, wafers 
were submerged in fresh running DI water for 5 min. Dehydration was held on a hot plate at $120^{\circ} \mathrm{C}$ for 10 min.

3. For ITO films, these layers were grown on a $\mathrm{Si}$ substrate, which was cleaned previously as it was described before. The films were annealed and then stored. Stored samples were cleaned only using acetone, methanol and DI water for $10 \mathrm{~min}$ each, and dehydrated on a hotplate at $120{ }^{\circ} \mathrm{C}$ for $10 \mathrm{~min}$.

The following paragraphs explain the processes of optimization of EBL on three different types of samples: (i) negative resist coated on $\mathrm{Si}$, (ii) negative resist coated on $\mathrm{GaN}$, and (iii) positive resist coated on GaN. These experiments were performed considering the different lattice constants for the $\mathrm{PhC}$ structures for each material, as they are described by Dawson et al. ${ }^{1}$

\subsubsection{Optimization of e-beam writing on $\mathrm{Si}$ with negative resist}

Si substrates were cleaned as it was explained above. Negative tone ma-N 2403 resist was spun onto the silicon substrate using a spinner that was set at $4500 \mathrm{rpm}$ for $45 \mathrm{sec}(\sim 270 \mathrm{~nm})$. The deposited resist was pre-baked on a hot plate at $90{ }^{\circ} \mathrm{C}$ for $3 \mathrm{~min}$ and subsequently left cooling to room temperature. The resist-coated substrate was diced into square pieces being ready to be exposed using the EBL system (JEOL 7600). EBL parameters exposure were (1) accelerating voltage of $30 \mathrm{kV}$, (2) field size of $100 \mu \mathrm{m}$, and (3) e-beam dose of $50 \mu \mathrm{C} / \mathrm{cm}^{2}$ to $120 \mu \mathrm{C} / \mathrm{cm}^{2}$. On the resist, dose characterization was performed. A $100 \mathrm{um}^{2}$ field with 4 internal PhC patterns with different doses was created with DesignCAD Express 16 (lattice constant $\mathrm{a}=260 \mathrm{~nm}$ and radius $=80 \mathrm{~nm}$ ). This cad file was loaded in the PC with NPGS to generate the run file for e-beam writing. CAD file and run file designs are discussed in Appendix A and $\mathrm{B}$, respectively. This dose-test pattern was written twice to cover a large range of doses $\left(50 \mu \mathrm{C} / \mathrm{cm}^{2}\right.$ to $120 \mu \mathrm{C} / \mathrm{cm}^{2}$ ). Figure 3-1 illustrates this characterization pattern on $\mathrm{Si}$. Thus, the right dose for this geometry of features, density of features, total structure area and substrate type was obtained. The development process of the exposed resist was realized in ma-D 525 solution for $70 \mathrm{sec}$, and the process was stopped by rinsing in DI water for $1 \mathrm{~min}$. Finally, the samples were dried very gently with a nitrogen gun (blowing with high pressure nitrogen can knock down easily the negative resist pillars on the substrate). These developed samples were deposited a thin film of Pt by a sputtering system. Samples were imaged using a SEM system. Each pattern on the sample was study to figure out the right dose for EBL. Figure 3-2 shows the results of the developed nanostructures. In Figure 3-2 (a) and (b) displays a $\mathrm{PhC}$ pattern overexposed, which results in features developed incompletely and pillar diameter greater than the one of the cad file; (c) and (d) displays a dose approximately right, pillars were developed all the way and its diameter has a $\sim 5 \%$ error; (e) and (f) depicts a pattern underexposed, features were fully 
developed although its diameter is way smaller than the one designed. The approximate right dose for $\mathrm{EBL}$ on this negative resist and Si substrate was found to be $70 \mu \mathrm{C} / \mathrm{cm}^{2}$.

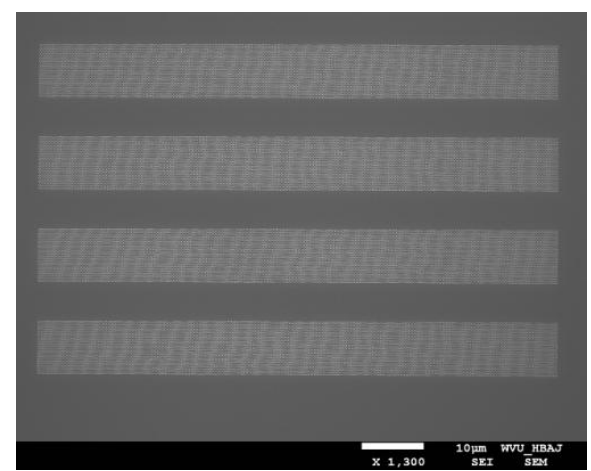

Figure 3-1: Top-view SEM micrograph of the dose-test pattern written and developed on Si.

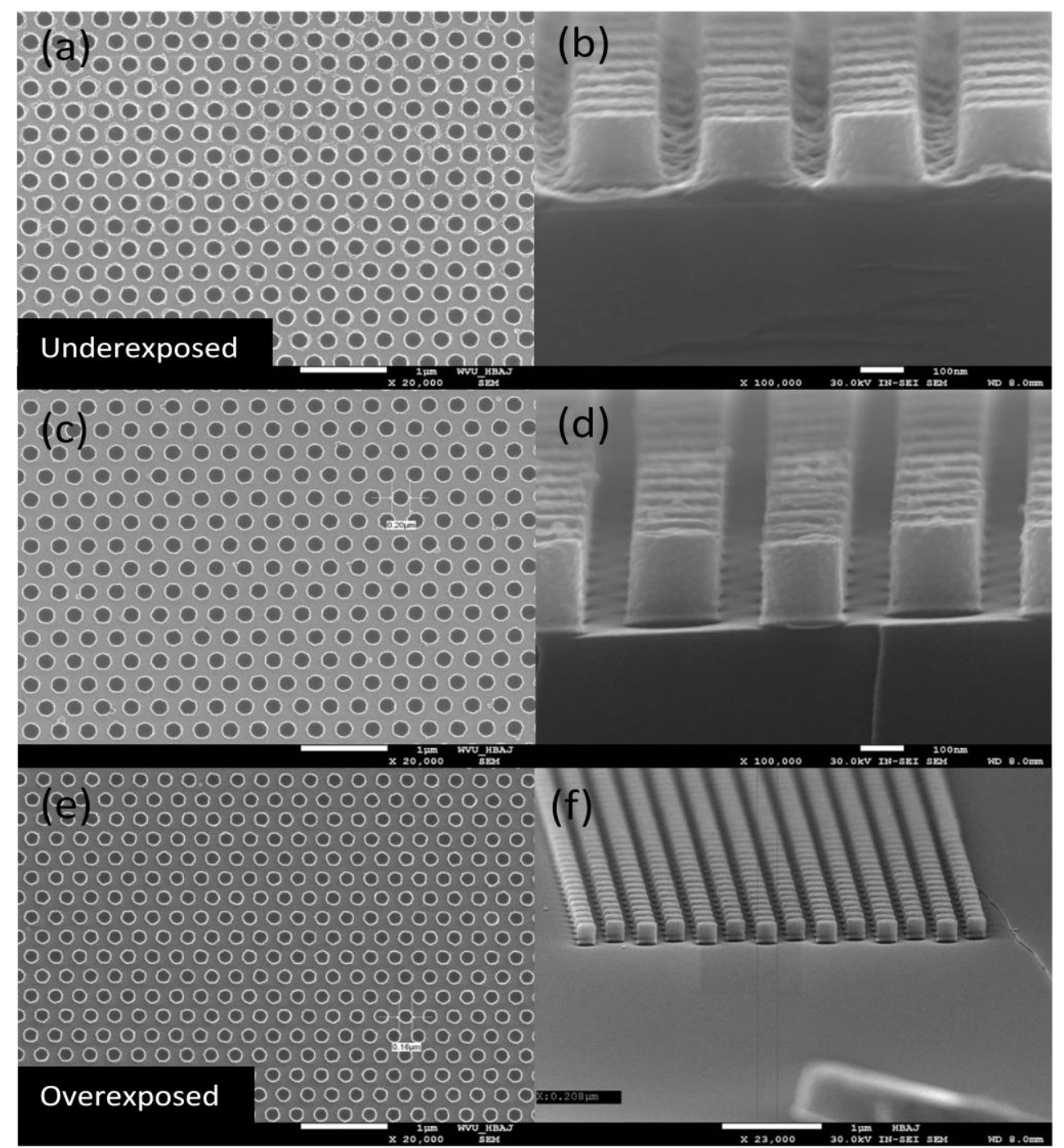

Figure 3-2: Top-view (left) and $45^{\circ}$-view (right) SEM micrographs of dose characterization with EBL for ma-N 2403: (a) and (b) underexposed patterns, (c) and (d) optimal exposure, (e) and (f) overexposed patterns. 


\subsubsection{Optimization of e-beam writing on $\mathrm{GaN}$ with positive resists.}

$\mathrm{GaN} \mathrm{PhC}$ structures are designed for use in the visible spectrum, so the lattice constant and airhole size are smaller than those of Si PhCs. Simulations suggest that GaN PhC structures with $\mathrm{r} \sim 100 \mathrm{~nm}$ and $\mathrm{r} / \mathrm{a} \sim 0.3$ have desirable photonic bandgaps for the biosensing applications. Also, GaN and Si have very different surface morphologies and optical properties. Therefore, their optimal EBL recipes are expected to be quite different. In this section, we will optimize and compare the e-beam writing processes on $\mathrm{GaN}$ with a positive tone resist PMMA 950 A and a negative tone resist ma-N in an effort to create high resolution PhC patterns with openings on the order of $\sim 100 \mathrm{~nm}$. .

Given that PMMA is not hard enough to undergo plasma etching, it needs to be used with an auxiliary mask, such as $\mathrm{SiO} 2$ or Ni. This will be described in detail when we develop the etch-back nanofabrication technique in section 3.3. Here our discussion is focused on pattern writing in the topmost PMMA resist.

$\mathrm{GaN}$ samples were previously cleaned and prebaked. A layer of $200 \mathrm{~nm}$ low stress layer of $\mathrm{SiO}_{2}$ was deposited by E-beam Evaporation (process parameters are listed in Table 3-2). Subsequently, a layer of $20 \mathrm{~nm} \mathrm{Ni}$ was deposited with the E-beam Evaporator. Process parameters are also listed in Table 3-2. A $\sim 80 \mathrm{~nm}$ thick PMMA $950 \mathrm{~K}$ e-beam resist layer was spin cast onto the samples at $4500 \mathrm{RPM}$ for $45 \mathrm{sec}$. The PMMA-layer was pre-baked in a hot plate for $75 \mathrm{sec}$ at $180{ }^{\circ} \mathrm{C}$. The $\mathrm{PhC}$ characterization pattern was generated by EBL using the JEOL 7600 system operating at $30 \mathrm{kV}$ and a beam current of $40 \mathrm{pA}$. These patterns contained features with lattice constant $\mathrm{a}=200 \mathrm{~nm}$ and radius $=50 \mathrm{~nm}$. The $\mathrm{PhC}$ characterization pattern, as shown in Figure 3-1, was written twice to cover a large range of doses $\left(250 \mu \mathrm{C} / \mathrm{cm}^{2}\right.$ to 460 $\mu \mathrm{C} / \mathrm{cm}^{2}$ ) for dose testing. Development process was carried out in: (1) a 1/3 mixture of methyl-isobutylketone (MIBK) and isopropanol (IPA) for $70 \mathrm{sec}$ with stirring, and (2) pure IPA bath for $30 \mathrm{sec}$. Samples were rinsed in DI water for $1 \mathrm{~min}$ and dried with a nitrogen gun. These patterns were coated with a thin sputterdeposited Pt for the purpose of SEM imaging. Figure 3-3 (a) shows a pattern underexposed at 250 $\mu \mathrm{C} / \mathrm{cm}^{2}$. Figure 3-3 (b) displays a pattern overexposed at $460 \mu \mathrm{C} / \mathrm{cm}^{2}$. And Figure 3-3 (c) and (d) illustrate approximately the right dose for these type of resist on this kind of substrate $\left(370 \mu \mathrm{C} / \mathrm{cm}^{2}\right)$.

\subsubsection{Optimization of e-beam writing on GaN with negative resists}

The experiment was setup in the same way as used for e-bema writing on $\mathrm{Si}$. In this case, the lattice parameters were shrank to $\mathrm{a}=200 \mathrm{~nm}$ and radius $=50 \mathrm{~nm}$. Figure 3-4 shows an SEM micrograph of the final result of EBL followed by deposition of a thin Pt film by sputtering. 


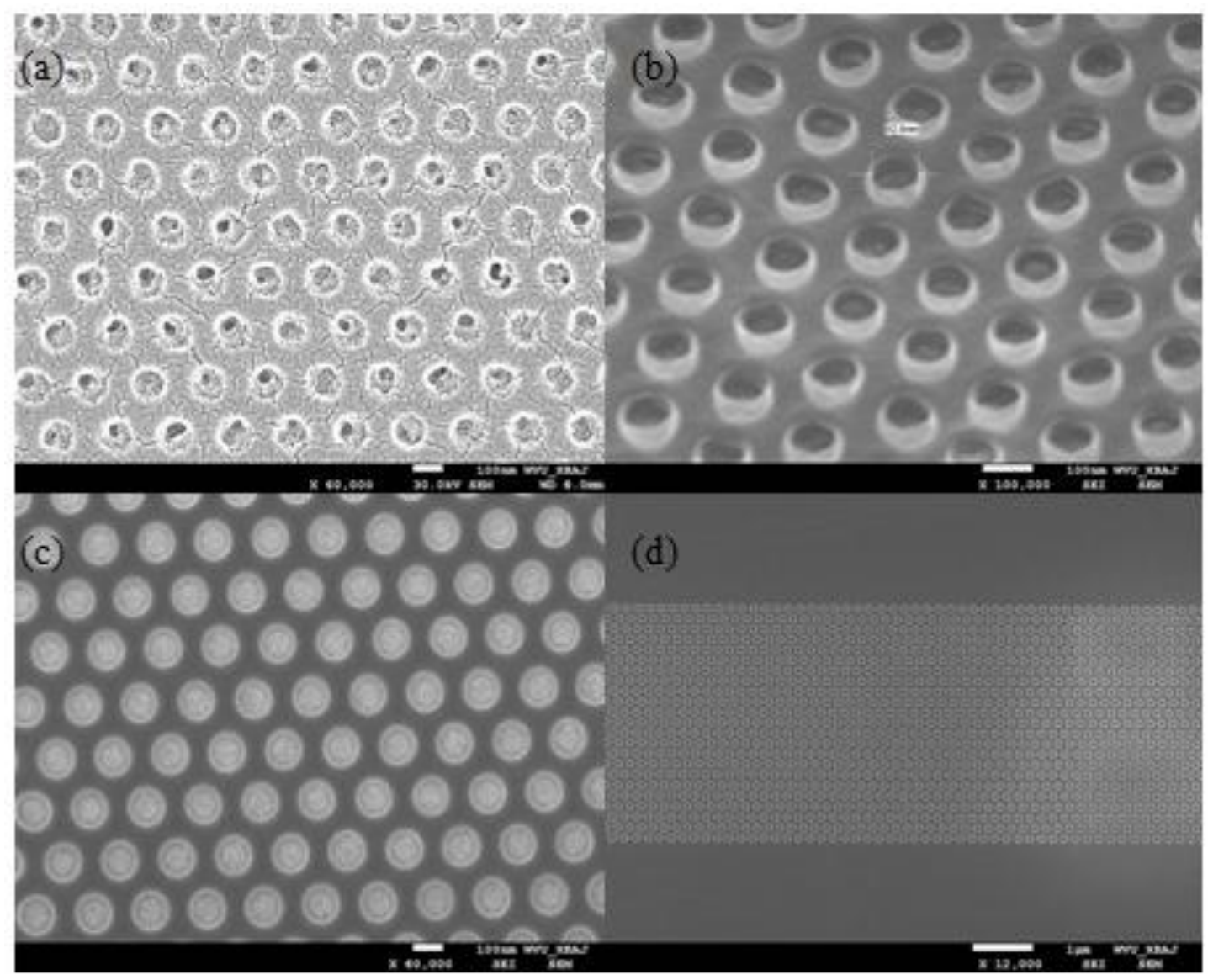

Figure 3-3: Top-view SEM micrographs of dose characterization with EBL for PMMA 950A: (a) underexposed pattern, (b) overexposed pattern, (c) and (d) optimal exposure $370 \mu \mathrm{C} / \mathrm{cm}^{2}$

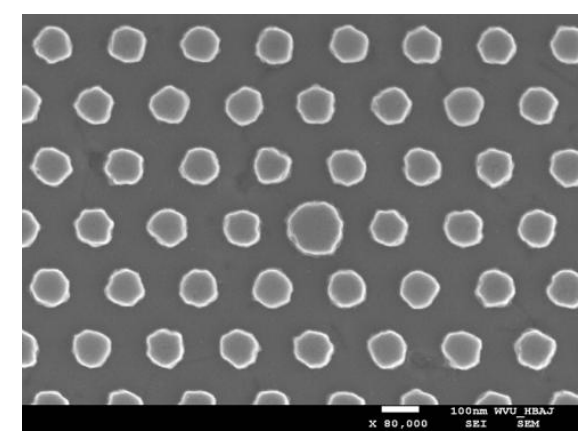

Figure 3-4: Top-view SEM micrographs of PhC pattern on $\mathrm{GaN}\left(65 \mu \mathrm{C} / \mathrm{cm}^{2}\right)$

As seen on Figure 3-3 (c), PMMA resist demonstrates high resolution PhC patterns. However, this film is only $\sim 80 \mathrm{~nm}$ thick. On the contrary, the negative resist ma-N 2403 presents poor resolution on the $\mathrm{PhC}$ patterns, but it is thicker than PMMA, $(\sim 270 \mathrm{~nm})$. This result can be attributed mostly to proximity 
effect, forward scattering of electrons due to resist thickness and the type of substrate. Backscattering and forward scattering are more noticeable in thick negative tone resists. To investigate this EBL limitation with the negative resist on $\mathrm{GaN}$ and such features, three different $\mathrm{PhC}$ structures, all with a 2D triangular lattice, were exposed in the ma-N 2403 resist. The specifications for these patterns are detailed in Table 3-1. The radius of the features was increased in order to diminish proximity effect.

\begin{tabular}{lccc} 
& Lattice constant $[\mathbf{n m}]$ & Hole radius [nm] & r/a \\
\hline Sample A & 1000 & 100 & 0.1 \\
\hline Sample B & 500 & 100 & 0.2 \\
\hline Sample C & 333 & 100 & 0.3
\end{tabular}

Table 3-1: Lattice parameters for PhC patterning on a negative resist using GaN substrate.

As it can be seen in Figure 3-5, feature resolution decreases as r/a ratio increases. For r/a $=0.1$ good resolution is obtained. In contrast, with $\mathrm{r} / \mathrm{a}=0.3$ resolution decreases. This can be explain as follows, as r/a ratio increases, the density of features also increases forcing closer beam exposures, which provokes the proximity effect to be more noticeable in the final structures. Then, there is a tradeoff between thickness of the resist and the resolution of features on the structures, when working with this kind of geometry. It is important to mention that thick masks and high resolution features are desirable for either subtractive or additive processes. 


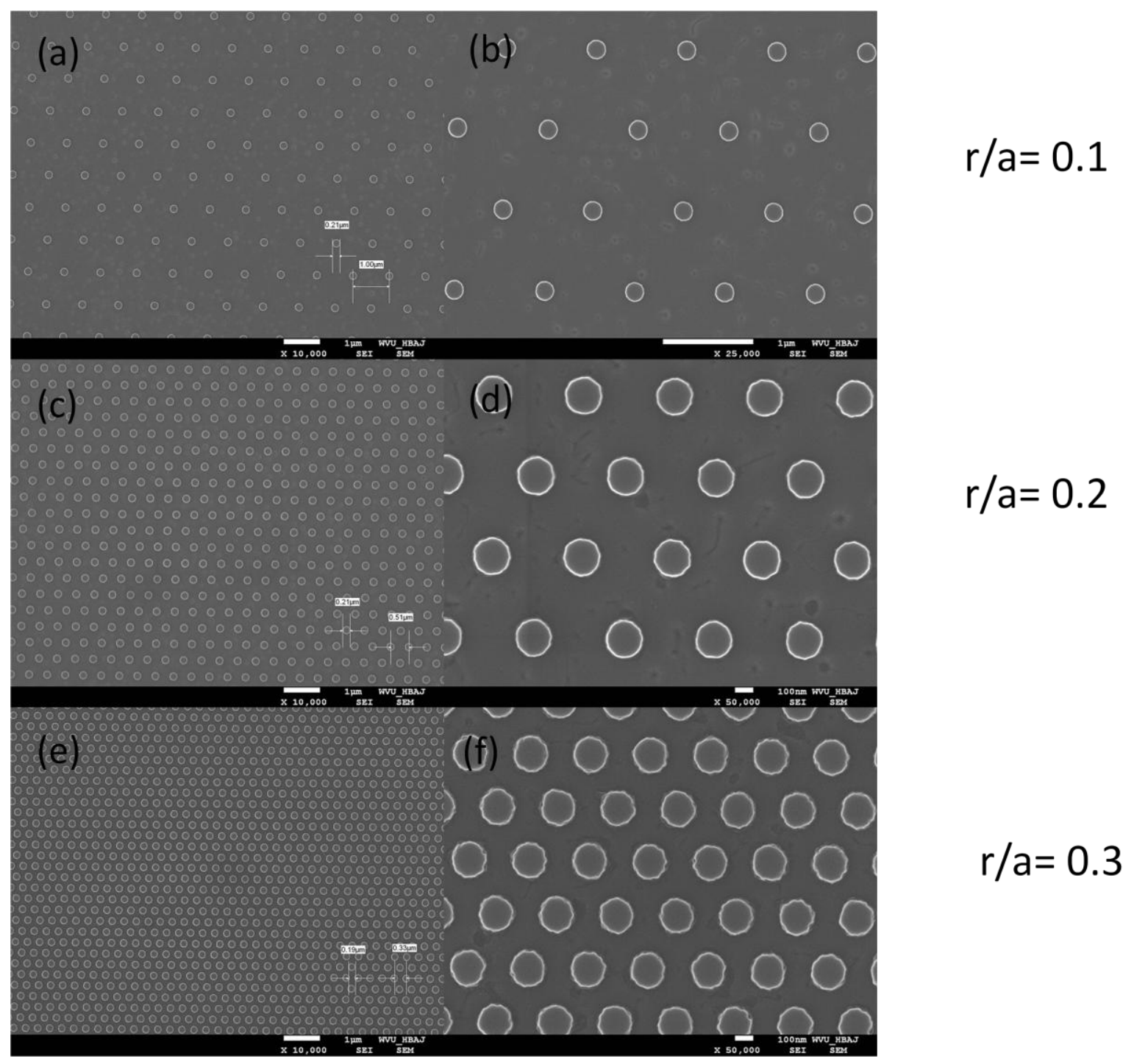

Figure 3-5: Top-view SEM micrographs of pattern resolution as a function of the lattice constant (a)-(d) r/a=0.1, (b)-(d) $\mathrm{r} / \mathrm{a}=0.2,(\mathrm{c})-(\mathrm{f}) \mathrm{r} / \mathrm{a}=0.3$

In order to make the $\mathrm{GaN} \mathrm{PhC}$ structures present a photonic bandgap on the visible spectrum, a ratio of $\mathrm{r} / \mathrm{a} \sim 0.25$ to 0.3 is required. For this reason, all the $\mathrm{GaN} \mathrm{PhC}$ structures written on the negative resist used writing parameters as follows: $\mathrm{a}=333 \mathrm{~nm}, \mathrm{r}=100 \mathrm{~nm}$, and an exposure dose of $65 \mu \mathrm{C} / \mathrm{cm}^{2}$. Developing process, for this negative resist, was fixed at $70 \mathrm{sec}$ in maD-525 solution. 


\subsection{COMPARISON OF LIFT-OFF AND ETCH-BACK PROCESSES FOR HARD MASK PATTERNING}

The transfer of $\mathrm{PhC}$ patterns into $\mathrm{Si}$ or $\mathrm{GaN}$ substrates requires the use of metal hard masks which have high etch resistance during plasma etching. The metal hard masks can be formed by either lift-off or etch-back. The former, also known as additive process, transfers patterns from resist to the metal mask by depositing a thin metal film on top of a prepatterned resist and lifting off the resist along with metal on top of it. The step leaves behind the metal that is deposited directly onto the substrate. The latter, also known as subtractive process, during which resist is spin coated on top of a thin metal layer and patterned. The pattern is then transfer to the metal layer using plasma etching. Here we compare the patterning of $\mathrm{Ni}$ hard masks using these two techniques.

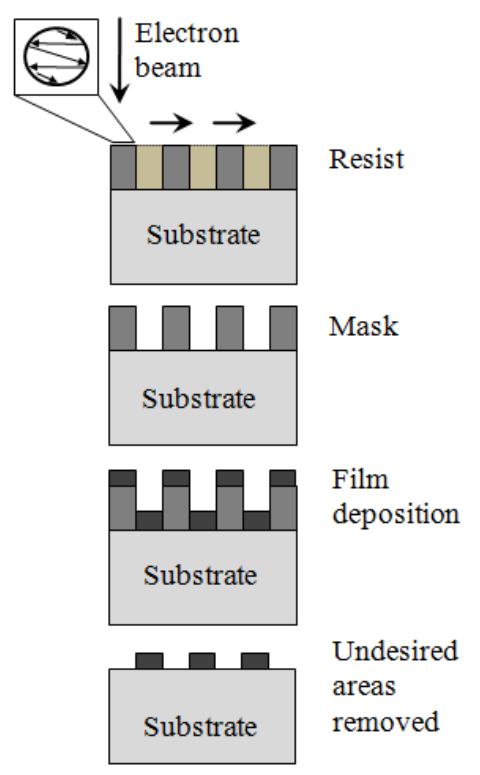

Figure 3-6: Process flow for lift-off.

\subsubsection{Hard mask patterning by Lift-off}

This is a simple, easy method for pattern transfer. A pattern is defined on a substrate using electron beam resist and EBL. A metal is blanket-deposited all over the substrate, covering the electron beam resist and areas where the resist has been cleared. During lift-off, the resist under the film is removed with solvents, leaving behind only the final patterned metal mask. This technique can be carried out if the following conditions are met: 
- During the metal film deposition, the substrate does not reach temperatures high enough to deform the resist patterns.

- Outgassing from the resist in the deposition chamber has a limited impact on the quality of the deposited metal film.

- Adhesion of the deposited metal film on the substrate is very good.

- The thickness of the metal should be preferably kept less than $1 / 3$ of the total resist thickness to facilitate the liftoff, whereas it must be thick enough for the subsequent pattern transfer into the substrate.

Figure 3-6 (a) shows a flow diagram to demonstrate how lift-off technique takes place on a silicon substrate. Wafers were cleaned and prebaked, previously. Negative e-beam resist (ma-N 2403) is spun onto the substrate and exposed with EBL. The exposed substrate is developed in ma-D525. Samples with defined patterns are taken to an electron beam evaporation system to deposited $\mathrm{Ni}$ as a metal mask. Liftoff can be accomplished, soaking the samples in acetone, and sonicating them to wash away any resist leftover on the substrate. The length of time for lift-off will depend on the film quality (generally, the higher the film quality, the more impermeable it is and the longer it will take to lift-off). It is recommended, keep the substrate immersed in acetone until all the film has been lifted-off because once particles dry on the substrate, they become hard to be removed. Finally, samples with Ni mask were ICP etched in $\mathrm{CF}_{4}$-based plasma. Mask removal was performed with $\mathrm{Ni}$ wet etchant. Figure 3-7 are SEM images to show the steps of fabrication based on lift-off technique above described. 


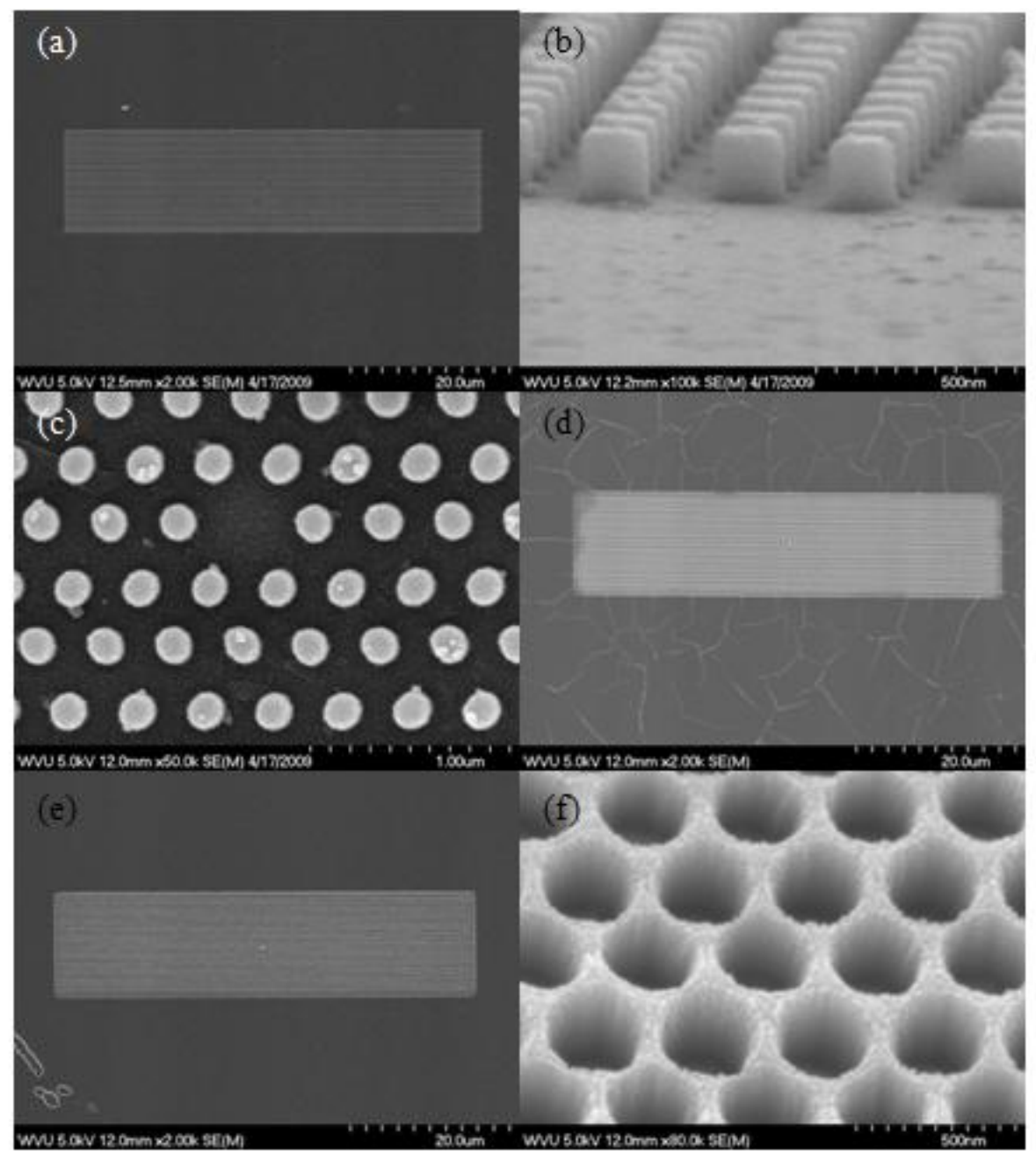

Figure 3-7: Lift-off technique, (a), (b), (c) Top-view SEM micrograph of the patterns written on the negative resist by EBL, (d) Top-view SEM micrograph of the Ni metal mask after lift-off and ICP etching, (e) Top-view SEM micrograph of the whole $\mathrm{PhC}$ structure on $\mathrm{Si}$, (f) $45^{\circ}$-view SEM micrograph of the final $\mathrm{PhC}$ structures (parameters of fabrication non optimized).

\subsubsection{Hard mask patterning by Etch-back}

The etch-back technique uses etching processes to transfer patterns from resist to a metal mask layer (e.g. Ni or $\mathrm{Cr}$ ). This is possible only if the resist layer is relative thick and the metal has a good etch selectivity over the resist. Unfortunately, the e-beam resist must be thin to retain good resolution for pattern writing, and usually has a poor etch resistance in plasma. An alternative method is to use a metal/dielectric bilayer hard mask and employ a two-step etch back pattern transfer process. In this work, $\mathrm{Ni}$ and $\mathrm{SiO}_{2}$ were deposited on $\mathrm{GaN}$ as the bilayer mask. The resist pattern was first transferred to a $\mathrm{Ni}$ layer by plasma etching, and further transferred to a thick $\mathrm{SiO}_{2}$ layer. This approach takes the advantage of high selectivities of $\mathrm{SiO}_{2}$ over $\mathrm{Ni}$, so a very thin $\mathrm{Ni}$ can be used to facilitate the first pattern transfer. Note that the metal layer is also critical for eliminating the charging effect during the initial e-beam 
writing. To optimize this process, detailed characterization of the etching behaviors of the e-beam resist, metal, $\mathrm{SiO} 2$ and $\mathrm{GaN}$ is required.

For the first layer, three different materials (three samples) were chosen to investigate the selectivity between these materials and $\mathrm{GaN}$, when they were etched in $\mathrm{BCl}_{3} / \mathrm{Cl}_{2}$ based plasma. These materials included: (i) $\mathrm{Si}_{3} \mathrm{~N}_{4}$ grown by PECVD, (ii) $\mathrm{SiO}_{2}$ grown by PECVD, and (iii) $\mathrm{SiO}_{2}$ grown by e-beam evaporation. Table 3-2 shows the parameters of the growing processes of each material. The as-deposit samples were cut into pieces and partially mask with photo resist AZ4400 for dry etching study. The samples were mounted using a thermal conductive paste on a Si wafer and then ICP etched at a pressure of 10 mTorr, RIE power $150 \mathrm{~W}$, ICP power $400 \mathrm{~W}$, and different percentages of $\mathrm{BCl}_{3}$ in the $\mathrm{BCl}_{3} / \mathrm{Cl}_{2}$ mix. Total gas flow was fixed at $30 \mathrm{sccm}$. Figure 3-8 shows the etching rates for the different materials.

\begin{tabular}{|c|c|c|c|c|c|c|c|c|c|c|c|}
\hline Material & $\begin{array}{l}\text { Growing } \\
\text { method }\end{array}$ & Temp & Pressure & $\mathrm{NH}_{3}$ & $\mathbf{N}_{2} \mathrm{O}$ & $\mathrm{SiH}_{4}$ & $\mathbf{O}_{2}$ & $\begin{array}{c}\text { RIE } \\
\text { power }\end{array}$ & $\begin{array}{c}\text { Substrate } \\
\text { Rotation }\end{array}$ & $\begin{array}{c}\text { Time } \\
\text { or } \\
\text { dep } \\
\text { rate }\end{array}$ & Thickness \\
\hline $\mathrm{SiO}_{2}$ & PECVD & $300^{\circ} \mathrm{C}$ & 1 Torr & & $\begin{array}{c}710 \\
\mathrm{sccm}\end{array}$ & $\begin{array}{c}170 \\
\text { sccm }\end{array}$ & I & $20 \mathrm{~W}$ & l & $5 \mathrm{~min}$ & $\sim 200 \mathrm{~nm}$ \\
\hline $\mathrm{Si}_{3} \mathrm{~N}_{4}$ & PECVD & $300^{\circ} \mathrm{C}$ & 1 Torr & $\begin{array}{c}35 \\
\mathrm{sccm}\end{array}$ & & $\begin{array}{r}400 \\
\text { sccm }\end{array}$ & I & $20 \mathrm{~W}$ & I & $\begin{array}{c}15 \\
\min \end{array}$ & $\sim 300 \mathrm{~nm}$ \\
\hline $\mathrm{SiO}_{2}$ & Evaporation & $150^{\circ} \mathrm{C}$ & $\begin{array}{l}0.01 \\
\text { mTorr }\end{array}$ & I & l & l & $\begin{array}{c}20 \\
\text { sccm }\end{array}$ & l & $20 \mathrm{RPM}$ & $\begin{array}{c}0.2 \\
{ }^{\circ} \mathrm{A} / \mathrm{sec}\end{array}$ & $\sim 200 \mathrm{~nm}$ \\
\hline $\mathrm{Ni}$ & Evaporation & $80{ }^{\circ} \mathrm{C}$ & $\begin{array}{c}0.01 \\
\text { mTorr }\end{array}$ & I & I & l & l & I & 20 RPM & $\begin{array}{c}0.2 \\
{ }^{\circ} \mathrm{A} / \mathrm{sec}\end{array}$ & $\sim 20 \mathrm{~nm}$ \\
\hline ITO & Evaportaion & $70{ }^{\circ} \mathrm{C}$ & $\begin{array}{c}0.01 \\
\text { mTorr }\end{array}$ & l & l & l & $\begin{array}{c}20 \\
\text { sccm }\end{array}$ & l & 20 RPM & $\begin{array}{c}0.2 \\
{ }^{\circ} \mathrm{A} / \mathrm{sec}\end{array}$ & $\sim 260 \mathrm{~nm}$ \\
\hline
\end{tabular}

Table 3-2: Growing parameter for PECVD and E-beam evaporation

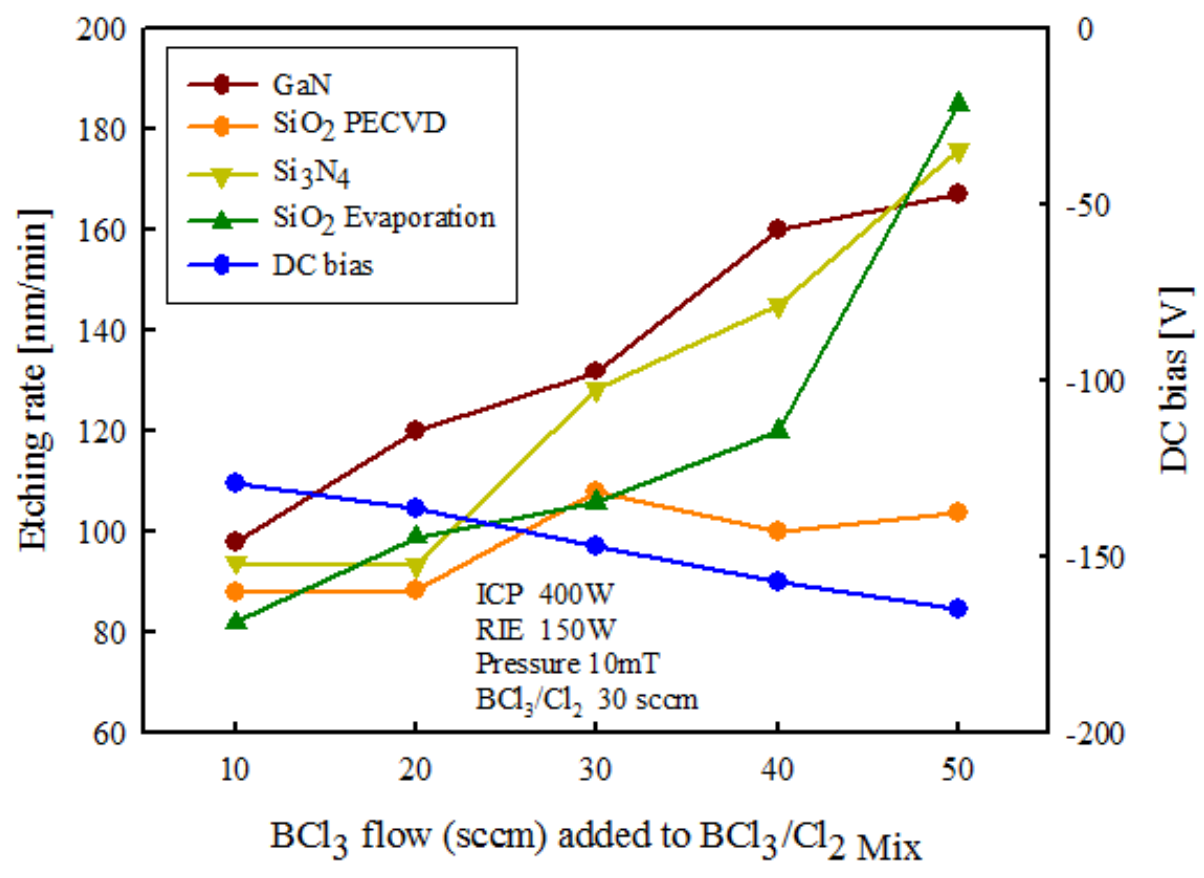

Figure 3-8: Etching rates of $\mathrm{SiO}_{2}$ and $\mathrm{Si}_{4} \mathrm{~N}_{3}$ grown by PECVD and Evaporation. 
As it can be seen in Figure 3-8, $\mathrm{SiO}_{2}$ grown by both methods presents low etch rate compared with $\mathrm{Si}_{3} \mathrm{~N}_{4}$ grown by PECVD and GaN. This means, the selectivity of $\mathrm{SiO}_{2}$ to $\mathrm{GaN}$ is higher than the one of the $\mathrm{Si}_{3} \mathrm{~N}_{4}$, what sets $\mathrm{SiO}_{2}$ as the best option as a hard mask. Though, $\mathrm{SiO}_{2}$ grown by e-beam evaporation was used due to its film quality.

For the second layer, $\mathrm{Ni}$ and $\mathrm{Cr}$ metals were considered because they etch very slowly in $\mathrm{CF}_{4}$ or $\mathrm{Cl}_{2}$ plasmas. However, Cr starts sputtering at high RIE powers (100 W and higher). ${ }^{50}$ Then, Ni metal was chosen as the best option as a hard mask. ICP etching characterization of $\mathrm{Ni}$ was performed using different plasma chemistries including $\mathrm{Ar}$ and $\mathrm{BCl}_{3}$. Two samples were deposited $\mathrm{Ni}$ with the e-beam evaporator according to the parameters shown in Table 3-2. ICP etching was performed with parameters described in Table 3-3. Finally, $\mathrm{SiO}_{2}$ grown by e-beam evaporation was ICP etched in $\mathrm{CF}_{4} / \mathrm{O}_{2}$ plasma to determine its etching rate. Table 3-3 shows also the etching rates for $\mathrm{Ni}$ and $\mathrm{SiO}_{2}$ for the different plasma chemistries. From Table 3-3, the difference of etching rates of $\mathrm{Ni}$ in the different plasmas was very small, which indicates that any plasma can be used for etching. However, $\mathrm{BCL}_{3}$ erodes PMMA much faster than Ar does. Therefore, Ar plasma was used to etch Ni films.

\begin{tabular}{|ll|llllll} 
Material & $\begin{array}{l}\text { Plasma } \\
\text { chemistry }\end{array}$ & Pressure & Gas flow & $\begin{array}{l}\text { RIE } \\
\text { power }\end{array}$ & $\begin{array}{l}\text { ICP } \\
\text { power }\end{array}$ & $\begin{array}{l}\text { Etching } \\
\text { rate }\end{array}$ & $\begin{array}{l}\text { Bias } \\
\text { Voltage }\end{array}$ \\
\hline $\mathrm{SiO}_{2}$ & $\mathrm{CF}_{4} / \mathrm{O}_{2}$ & $10 \mathrm{mTorr}$ & $18 / 2 \mathrm{sccm}$ & $150 \mathrm{~W}$ & $400 \mathrm{~W}$ & $340 \mathrm{~nm} / \mathrm{min}$ & $-130 \mathrm{~V}$ \\
\hline $\mathrm{Ni}$ & $\mathrm{BCl}_{3}$ & $30 \mathrm{mTorr}$ & $20 \mathrm{sccm}$ & $150 \mathrm{~W}$ & $400 \mathrm{~W}$ & $28 \mathrm{~nm} / \mathrm{min}$ & $-180 \mathrm{~V}$ \\
\hline $\mathrm{Ni}$ & $\mathrm{Ar}$ & $30 \mathrm{mTorr}$ & $20 \mathrm{sccm}$ & $150 \mathrm{~W}$ & $400 \mathrm{~W}$ & $22 \mathrm{~nm} / \mathrm{min}$ & $-77 \mathrm{~V}$ \\
\hline
\end{tabular}

Table 3-3: Etching rates for $\mathrm{SiO}_{2}$ and $\mathrm{Ni}$ for different plasma chemistries.

The data obtained from these experiments allow the fabrication of $\mathrm{PhC}$ structures using etch-back technique. Next, a stepwise description of the PhC fabrication using etch-back technique is given. GaN substrate was cleaned and prebaked. $\mathrm{A} \mathrm{SiO}_{2}$ layer with a thickness of $\sim 200 \mathrm{~nm}$ on $\mathrm{GaN}$ by E-beam Evaporation (process parameters are listed in Table 3-2). The thickness of this layer is chosen such as to enable the final deep etching step in $\mathrm{GaN}$ considering the $\mathrm{SiO}_{2} / \mathrm{GaN}$ etching selectivity. $\sim 20 \mathrm{~nm}$ Ni film was deposited with the e-beam evaporator (process parameters are also listed in Table 3-2). A 80nm thick PMMA 950A layer was spin coated onto the substrates at $4500 \mathrm{RPM}$ for $45 \mathrm{sec}$ and post baked at $180^{\circ} \mathrm{C}$ for $75 \mathrm{sec}$ (hotplate). Exposure of the photonic crystal patterns was performed with a JEOL 7600 EBL system at its maximum acceleration voltage of $30 \mathrm{kV}$. Exposure dose was fixed at $360 \mu \mathrm{C} / \mathrm{cm}^{2}$, and probe current to $40 \mathrm{pA}$ for writing patterns containing features with lattice constant a $=200 \mathrm{~nm}$ and radius $=50 \mathrm{~nm}$. An area of $2 \mathrm{~mm} \times 100 \mu \mathrm{m}$ was covered placing PhC patterns, one after each other horizontally. 
After exposure, samples were developed in MIBK:IPA 1:3 for 70sec, $20 \mathrm{sec}$ in IPA and rinsed with DI water for $1 \mathrm{~min}$. Then, PMMA was cured in an oven at $110{ }^{\circ} \mathrm{C}$ for $30 \mathrm{~min}$. Thus samples were ready for the dry etching process. The PhC patterns on the resist were transferred into the Ni metal layer by ICP etching with Ar using parameters from Table 3-3. This was followed by $\mathrm{CF}_{4}$-based plasma etching (parameter described in Table 3-3), which etched the airholes into the $\mathrm{SiO}_{2}$ layer. The final dry etching step was done for $2 \mathrm{~min}$ in $\mathrm{BCL}_{3} / \mathrm{Cl}_{2}$ plasma with $\mathrm{Cl}_{2} / \mathrm{BCl}_{3}$ gas flow rate of 20/10 (sccm), 10mTorr pressure, 400W ICP power and 150W RIE power. This step transferred the airholes through the $\mathrm{SiO}_{2}$ into the GaN substrate. Once the dry etching was complete the remains of the surface mask were removed using a HF bath for 30sec. Figure 3-9 displays a flow diagram describing the process of PhC fabrication on $\mathrm{GaN}$ with etch-back technique.

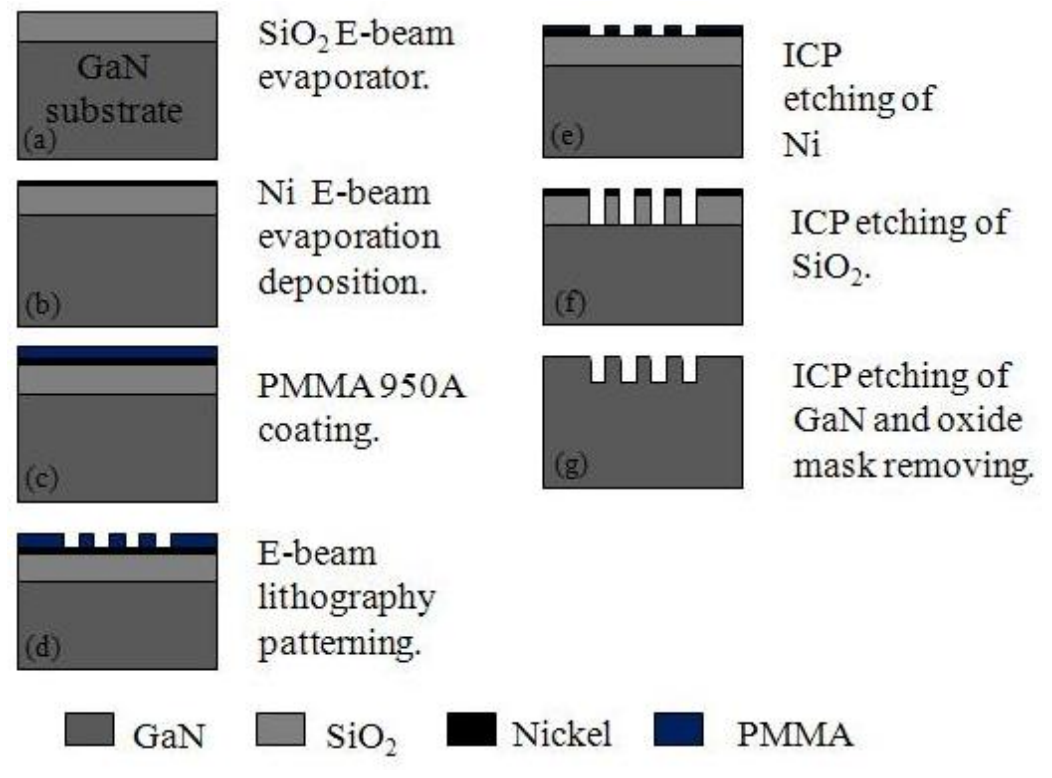

Figure 3-9: Process flow for the fabrication of PhC on GaN (etch-back approach)

Figure 3-10 shows the SEM images of the $\mathrm{PhC}$ fabrication. Etch-back is a popular technique for $\mathrm{PhC}$ fabrication due to the fact that renders deep features and vertical sidewalls. However with our equipment, this process ran into some problems that will be discussed later. Figure 3-10 (a) displays a top view of the PhC on PMMA; (b) shows a top view of the PhC on PMMA after curing process; (c) top view of the pattern transfer from PMMA into $\mathrm{Ni}$; (d) cross section of the $\mathrm{PhC}$ on the $\mathrm{Ni}$ mask. $\mathrm{SiO}_{2}$ layer and substrate are also visible; (e) pattern transfer from $\mathrm{Ni}$ to $\mathrm{SiO}_{2}, 45^{\circ}$ view; (f) pattern transfer from $\mathrm{SiO}_{2}$ to $\mathrm{GaN}$, $45^{\circ}$ view; (g) cross section of PhC on GaN after ICP etching; (h) top-view of final PhC structure on $\mathrm{GaN}$, after removing $\mathrm{SiO}_{2}$. As it is seen in this figure, the final $\mathrm{PhC}$ fabrication failed because of two issues: first, PMMA could not stand as hard mask even though it was previously cured, so after $\mathrm{Ar}^{+}$ bombardment, mask resolution degraded very much. Second, $\mathrm{SiO}_{2}$ could not stand either as a hard mask 
for $\mathrm{Cl}_{2} / \mathrm{BCl}_{3}$-based plasmas. Therefore, it seemed that PMMA curing should be realized at higher temperatures, and the $\mathrm{SiO}_{2}$ layer should be treated previously to the dry etching process.

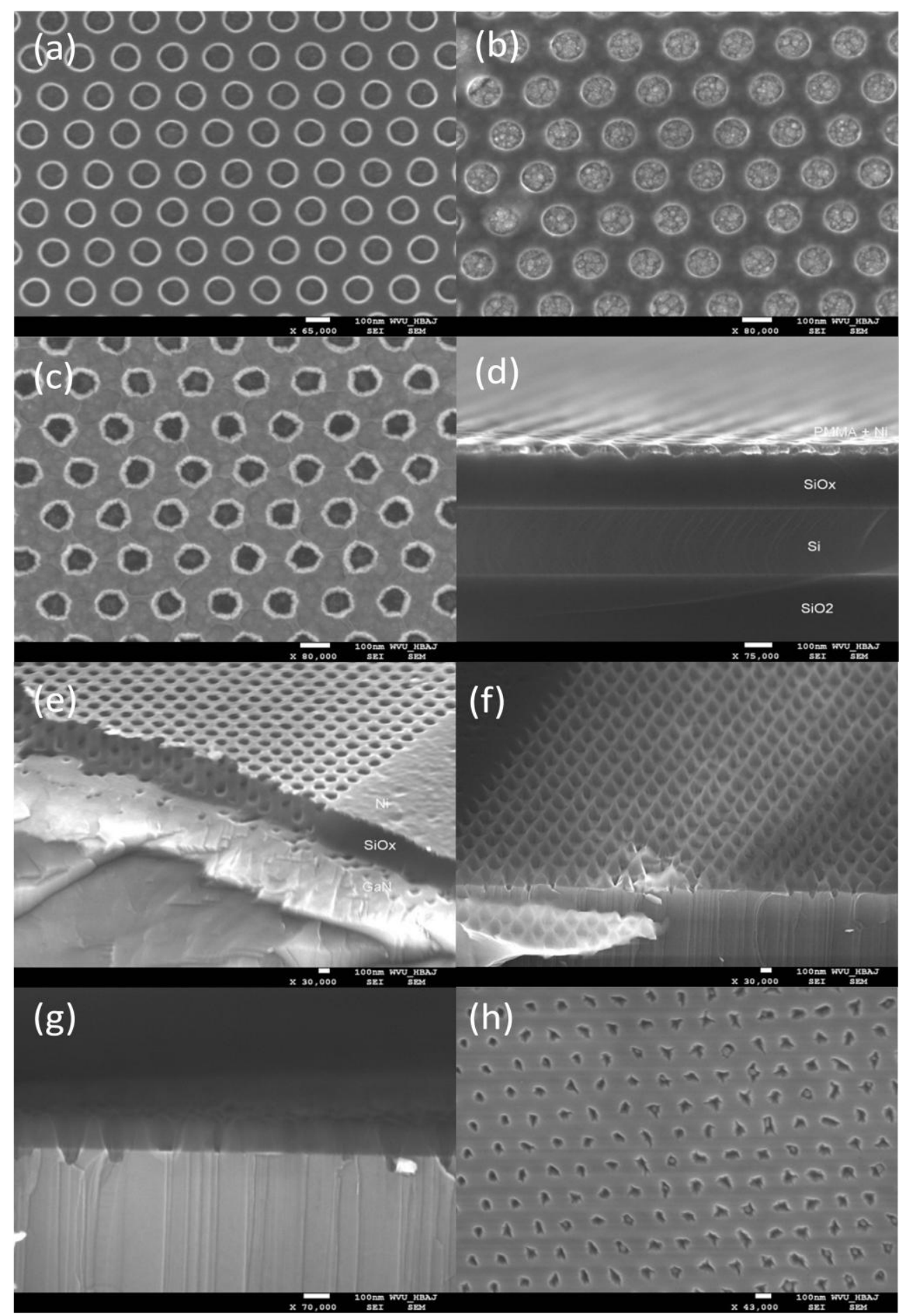

Figure 3-10: SEM micrographs of the PhC fabrication on GaN (etch-back): (a) PhC on PMMA, (b) cured PMMA, (c) ICP etching of $\mathrm{Ni}$, (d) cross section of $\mathrm{PhC}$ on $\mathrm{Ni}$, (e) ICP etching of $\mathrm{SiO}_{2}$, (f) ICP etching of $\mathrm{GaN}$, (g) cross section of $\mathrm{PhC}$ on $\mathrm{GaN}-$ $\mathrm{SiO}_{2}$ mask still on top of the substrate, (h) $\mathrm{SiO}_{2}$ mask removal (final structure on $\mathrm{GaN}$ ). 
To investigate the highest temperature for hardening the PMMA layer, before reflow starts altering the patterns, a set of three samples were written by EBL and then cured in an oven at $110{ }^{\circ} \mathrm{C}, 125{ }^{\circ} \mathrm{C}, 140$ ${ }^{\circ} \mathrm{C}$ for 30 min. SEM images reveal that PMMA reflows at temperatures higher than $120{ }^{\circ} \mathrm{C}$. Then, it has been assumed that somehow the temperature inside the ICP RIE system, while transferring the pattern from PMMA into $\mathrm{Ni}$, reached higher levels than $120{ }^{\circ} \mathrm{C}$ deforming the patterns. Figure 3-11 depicts how PMMA features deformed: (a) deformation of features on the edge of the PhC structure; (b) deformation of features in the center of the structure. This issue became a resolution limitation in the $\mathrm{PhC}$ fabrication with etch-back approach.

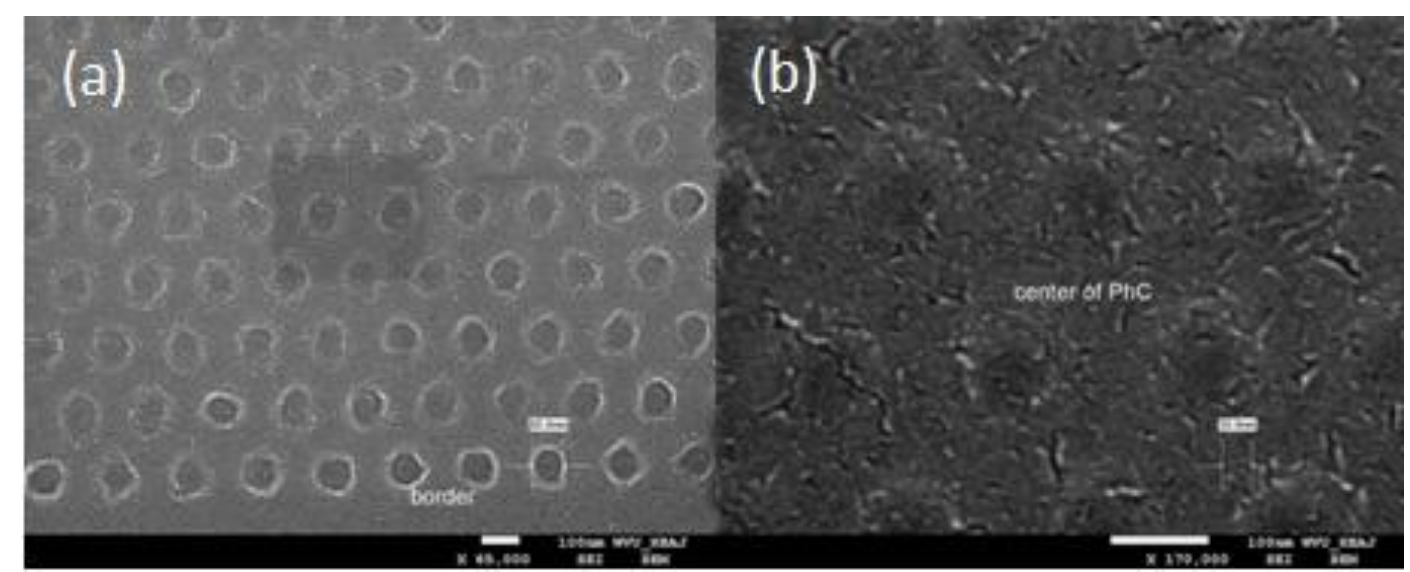

Figure 3-11: Top-view SEM micrographs of PMMA reflow at $140{ }^{\circ} \mathrm{C}$ : (a) edge of the PhC, (b) center of the PhC.

After analyzing this two nanofabrication techniques, it can be concluded that pattern transfer from a negative e-beam, based on lift-off technique, produced uniform $\mathrm{PhC}$ structures on Si substrate. However, it is not suitable for fabricating sub-100 nm PhC structures for GaN. Then this issue forces to increase the radius of the features and decrease a little bit the ratio of r/a in order to increase pattern resolution reducing proximity effect and keep the photonic bandgap still in the visible spectrum. Etch-back technique was not able to produce fine $\mathrm{PhC}$ structures due to equipment limitation (ICP chamber temperature higher than $120^{\circ} \mathrm{C}$ causes deformation on PMMA patterns).

\subsection{FABRICATION OF PHC STRUCTURES ON SI}

After the hard mask is formed by the lift-off process, the remaining step for Si PhC fabrication is plasma etching of Si for the final pattern transfer. Figure 3-12 shows a whole process flow carried out for fabricating photonic crystal structures on Si. Si substrates were cleaned using solvents and BOE. Negative tone ma-N 2403 resist was spun onto the silicon substrate using a spinner that was set at $4500 \mathrm{rpm}$ for 45 
$\sec (\sim 270 \mathrm{~nm})$. The deposited resist was pre-baked on a hot plate at $90{ }^{\circ} \mathrm{C}$ for $3 \mathrm{~min}$ and subsequently left cooling to room temperature. The resist-coated substrate was diced into square pieces being ready to be exposed using the EBL system (JEOL 7600). PhC structures were defined by EBL with parameters: (1) accelerating voltage of $30 \mathrm{kV}$, (2) field size of $100 \mu \mathrm{m}$, (3) probe current $40 \mathrm{pA}$, and (4) e-beam dose 70 $\mu \mathrm{C} / \mathrm{cm}^{2}$. PhC patterns contained features with lattice constant $\mathrm{a}=260 \mathrm{~nm}$ and radius $=84 \mathrm{~nm}$. An area of 2 $\mathrm{mm} \times 100 \mu \mathrm{m}$ was covered placing PhC patterns, one after each other horizontally. The development process of the exposed resist was realized in ma-D 525 solution for $70 \mathrm{sec}$, and the process was stopped by rinsing in DI water for $1 \mathrm{~min}$. The samples were dried very gently with a nitrogen gun. Developed samples were deposited $\sim 30 \mathrm{~nm}$ of $\mathrm{Ni}$ by e-beam evaporation. Deposition parameters are listed in Table 3-2. Once the samples were deposited $\mathrm{Ni}$, these were dip into acetone for lift-off for $30 \mathrm{~min}$. The pattern transfer into Si substrate was carried out using an ICP RIE system in $\mathrm{CF}_{4} / \mathrm{O}_{2}$ based plasma with gas flow rates of 18/2 sccm (standard cubic centimeter per minute), at a Pressure of 10mTorr, ICP power of 400W, and RIE power of $150 \mathrm{~W}$ for $1 \mathrm{~min}$. these samples were wet etched with $\mathrm{Ni}$ etchant for $15 \mathrm{~min}$ in order to remove the metal mask left. These samples were cleaved across the PhC structures patterns in order to study its cross section. Finally, cleaved samples were coated with a thin sputter-deposited Pt for the purpose of SEM imaging. Figure 3-13 shows the final structures on Silicon: (a), (b) top view of Si PhC structure an its nanocavity defect (c), (d) cross section of the structure (study of sidewall profiles).
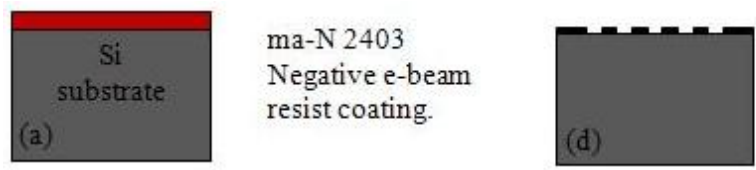

Metal lift-off.

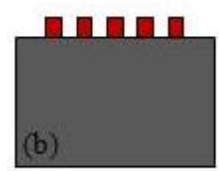

E-beam lithography patterning.

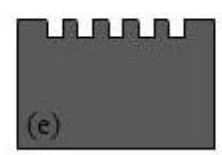

ICP etching

of $\mathrm{Si}$ and metal mask removing.

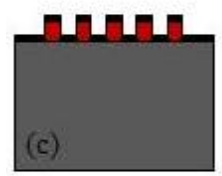

E-beam evaporation deposition of $\mathrm{Ni}$.

$\square$ Resist $\square$ Silicon $\square$ Nickel

Figure 3-12: Process flow for the fabrication of $\mathrm{PhC}$ on $\mathrm{Si}$ 


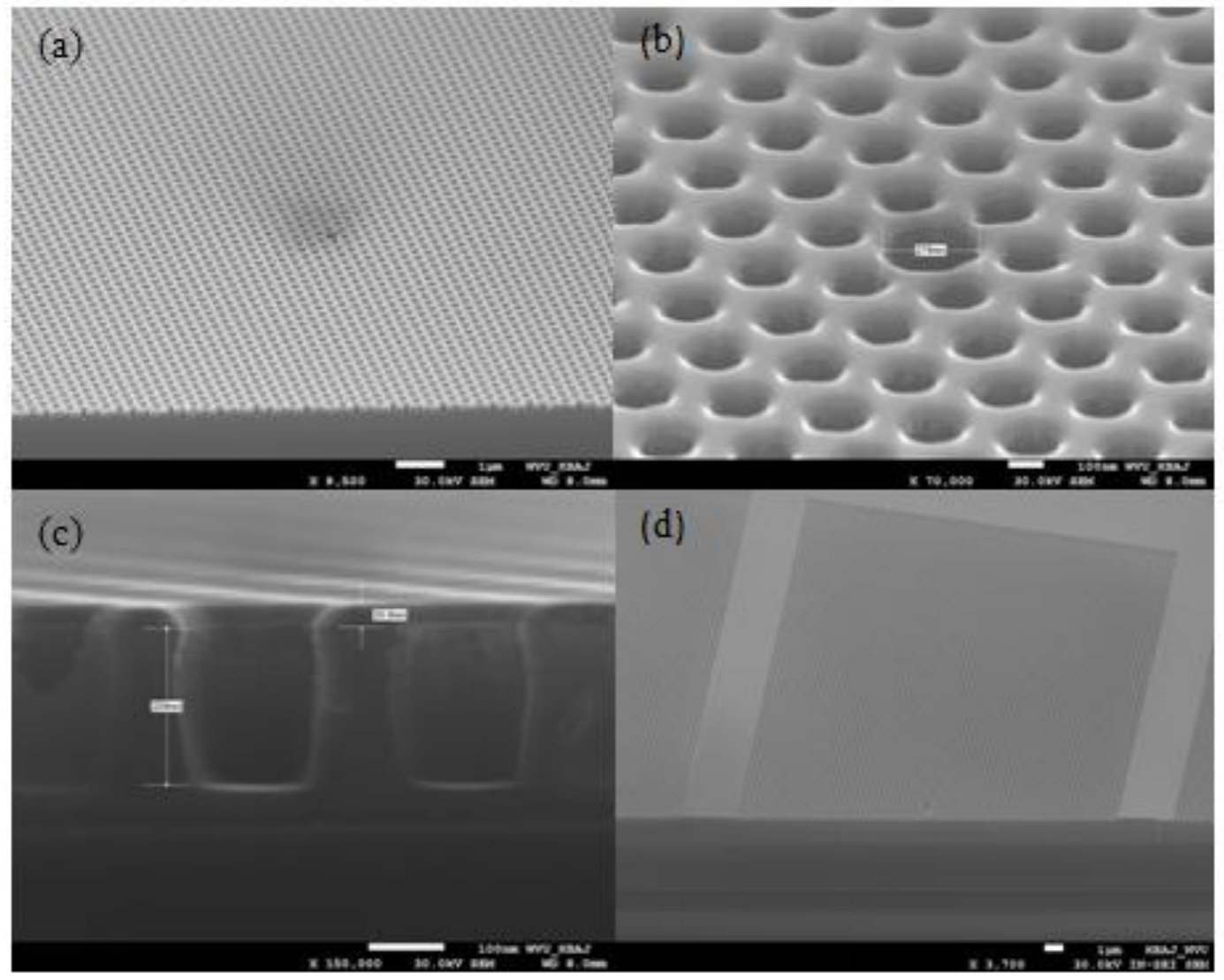

Figure 3-13: SEM micrographs of the fabrication of the $\mathrm{PhC}$ on $\mathrm{Si}$ : (a) $45^{\circ}$ view of $\mathrm{PhC}$ on $\mathrm{Si}$, (b) $45^{\circ}$ view of nanocavity in the $\mathrm{PhC}$ structure, (c) cross section of $\mathrm{PhC}$-sidewall profiles, (d) $45^{\circ}$ view of the whole $\mathrm{PhC}$ structure.

\begin{tabular}{cccc}
\multicolumn{2}{c}{ Hole Diameter } \\
Material & Design $[\mathrm{nm}]$ & Measured $[\mathrm{nm}]$ & Error percentage $[\%]$ \\
\hline Silicon & 168 & 171 & 1.785714286 \\
\hline
\end{tabular}

The table above shows final measurements of the holes in the $\mathrm{PhC}$ structure on Silicon, and the computations of its respective error compared with the dimensions of the CAD design file.

\subsection{FABRICATION OF PHC STRUCTURES ON GaN}

During the fabrication of GaN PhC structures, the hard masks were patterned by either simple lift off or a modified two-step pattern transfer method. The latter combines lift off and etch back, two pattern transfers from resist to metal and dielectric masks. We compared these two processes in order to identify a better method which may yield final $\mathrm{GaN}$ PhC structures with good pattern uniformity as well as desirable airhole size and sidewall profiles. 


\subsubsection{Fabrication of PhCs on GaN using hard masks formed by lift-off}

Figure 3-14 is a summary of each step of the PhC fabrication on GaN using lift off technique and a single layer as a hard mask (Ni). GaN substrates were cleaned and dehydrated as it was explained above. Negative tone ma-N 2403 resist was spun onto the GaN substrate using a spinner that was set at $4500 \mathrm{rpm}$ for $45 \mathrm{sec}(\sim 270 \mathrm{~nm})$. The deposited resist was pre-baked on a hot plate at $90{ }^{\circ} \mathrm{C}$ for $3 \mathrm{~min}$ and subsequently left to cool to room temperature. The resist-coated substrate was diced into two square pieces being ready to be exposed using the EBL system (JEOL 7600). PhC structures were defined by EBL with parameters: (1) accelerating voltage of $30 \mathrm{kV}$, (2) field size of $100 \mu \mathrm{m}$, (3) probe current $40 \mathrm{pA}$, and (4) e-beam dose of $65 \mu \mathrm{C} / \mathrm{cm}^{2}$. PhC patterns contained features with lattice constant a $=333 \mathrm{~nm}$ and radius $=100 \mathrm{~nm}$. An area of $2 \mathrm{~mm} \times 100 \mu \mathrm{m}$ was covered placing PhC patterns, one after each other horizontally. The development process of the exposed resist was realized in ma-D 525 solution for $70 \mathrm{sec}$, and the process was stopped by rinsing in DI water for $1 \mathrm{~min}$. The samples were dried very gently with a nitrogen gun. Developed samples were deposited $\sim 95 \mathrm{~nm}$ Ni by e-beam evaporation. Deposition parameters are listed in Table 3-2. Once the samples were deposited $\mathrm{Ni}$, these were dip into acetone for lift-off for $30 \mathrm{~min}$. The pattern transfer into GaN substrate was performed in an ICP RIE system using two different plasmas: (i) $\mathrm{BCl}_{3} / \mathrm{Cl}_{2}$ (gas rate $=27 / 3 \mathrm{sccm}$ ), and (ii) $\mathrm{Cl}_{2} / \mathrm{Ar}$ (gas rate $=20 / 10 \mathrm{sccm}$ ) to investigate which plasma chemistry gives better structures and depths. For both process all the parameters were fixed at $10 \mathrm{mT}$ Torr pressure, $400 \mathrm{~W}$ ICP power, and 150W RIE power. Ni etchant removed any Ni mask left on the substrate. Finally, samples were cleaved in the middle of the $\mathrm{PhC}$ pattern, and then a thin film of Pt was sputtered for SEM imaging (cross section study). Figure 3-15 shows SEM images all of the fabrication stages using lift-off technique.

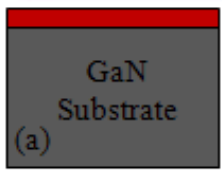

ma-N 2403

Negative e-beam resist coating.

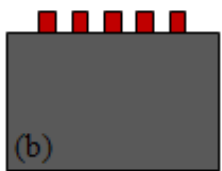

E-beam lithography patteming.

\section{E-beam} evaporation deposition of $\mathrm{Ni}$.
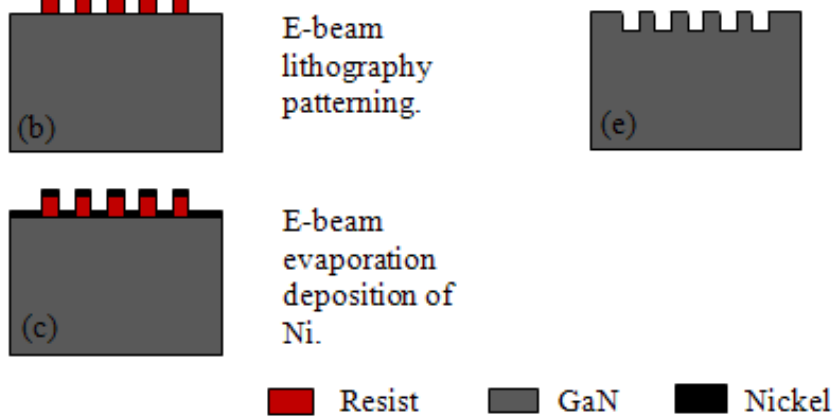

Metal lift-off.

ICP etching

of $\mathrm{GaN}$ and metal mask

removing.

Figure 3-14: Process flow for the fabrication of $\mathrm{PhC}$ on $\mathrm{GaN}$ (lift-off approach) 
Figure 3-16 shows the cross sections of sidewall profiles of $\mathrm{PhC}$ structures on $\mathrm{GaN}$ : (a) and (b) are non optimized ICP etching process on the fabrication. Poor verticality on sidewall was obtained because metal mask was too thin (metal mask used to be $30 \mathrm{~nm}$ thick). Hence, the hard mask run out before the etching process was finished. Figure 3-14 (c) and (d) shows final sidewalls and depths of PhC structure for $\mathrm{Cl}_{2} / \mathrm{Ar}$ based plasma etch process and finally Figure 3-14 (e) and (f) displays final sidewalls and depths of PhC structures for $\mathrm{Cl}_{2} / \mathrm{BCl}_{3}$ based plasma. Table 3-4 summarizes parameters of ICP etching operation, etching rates, and selectivities for the different plasma chemistries when etching GaN.

\begin{tabular}{|clcccccc}
$\begin{array}{c}\text { Plasma } \\
\text { chemistry }\end{array}$ & Pressure & Gas flow & $\begin{array}{c}\text { RIE } \\
\text { power }\end{array}$ & $\begin{array}{c}\text { ICP } \\
\text { power }\end{array}$ & $\begin{array}{c}\text { Etching } \\
\text { rate }\end{array}$ & $\begin{array}{c}\text { Selectivity } \\
\begin{array}{c}\text { Sidewall } \\
\text { angle }\end{array}\end{array}$ \\
\hline $\mathrm{Cl}_{2} / \mathrm{BCl}_{3}$ & $10 \mathrm{mTorr}$ & $27 / 3 \mathrm{sccm}$ & $150 \mathrm{~W}$ & $400 \mathrm{~W}$ & $85 \mathrm{~nm} / \mathrm{min}$ & 16 & $87.6^{\circ}$ \\
\hline $\mathrm{Cl}_{2} / \mathrm{Ar}$ & $10 \mathrm{mTorr}$ & $20 / 10 \mathrm{sccm}$ & $150 \mathrm{~W}$ & $400 \mathrm{~W}$ & $70 \mathrm{~nm} / \mathrm{min}$ & 10 & $83.3^{\circ}$ \\
\hline
\end{tabular}

Table 3-4: Etching rates and selectivities for ICP etching of GaN in different plasma chemistries. 


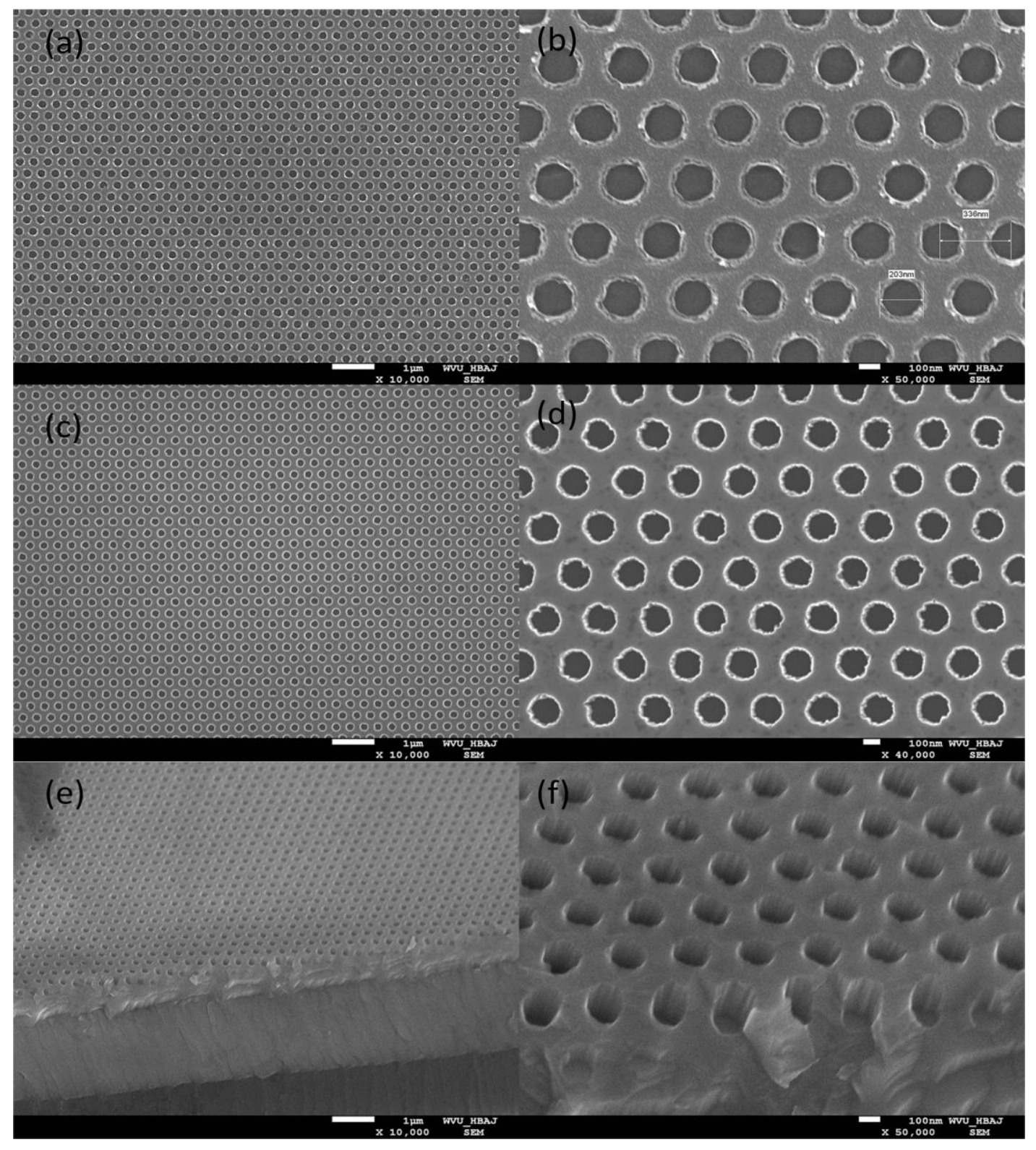

Figure 3-15: SEM micrographs of the PhC structure on GaN, lift-off process (a) top view of PhC on the resist layer, (b) top view of Ni metal mask after lift-off, (c) top view of PhC pattern transfer from Ni mask into GaN, (d) top view of final PhC structure on $\mathrm{GaN}$, (e)-(f) final $\mathrm{PhC}$ structures on $\mathrm{GaN}, 45^{\circ}$ view. 


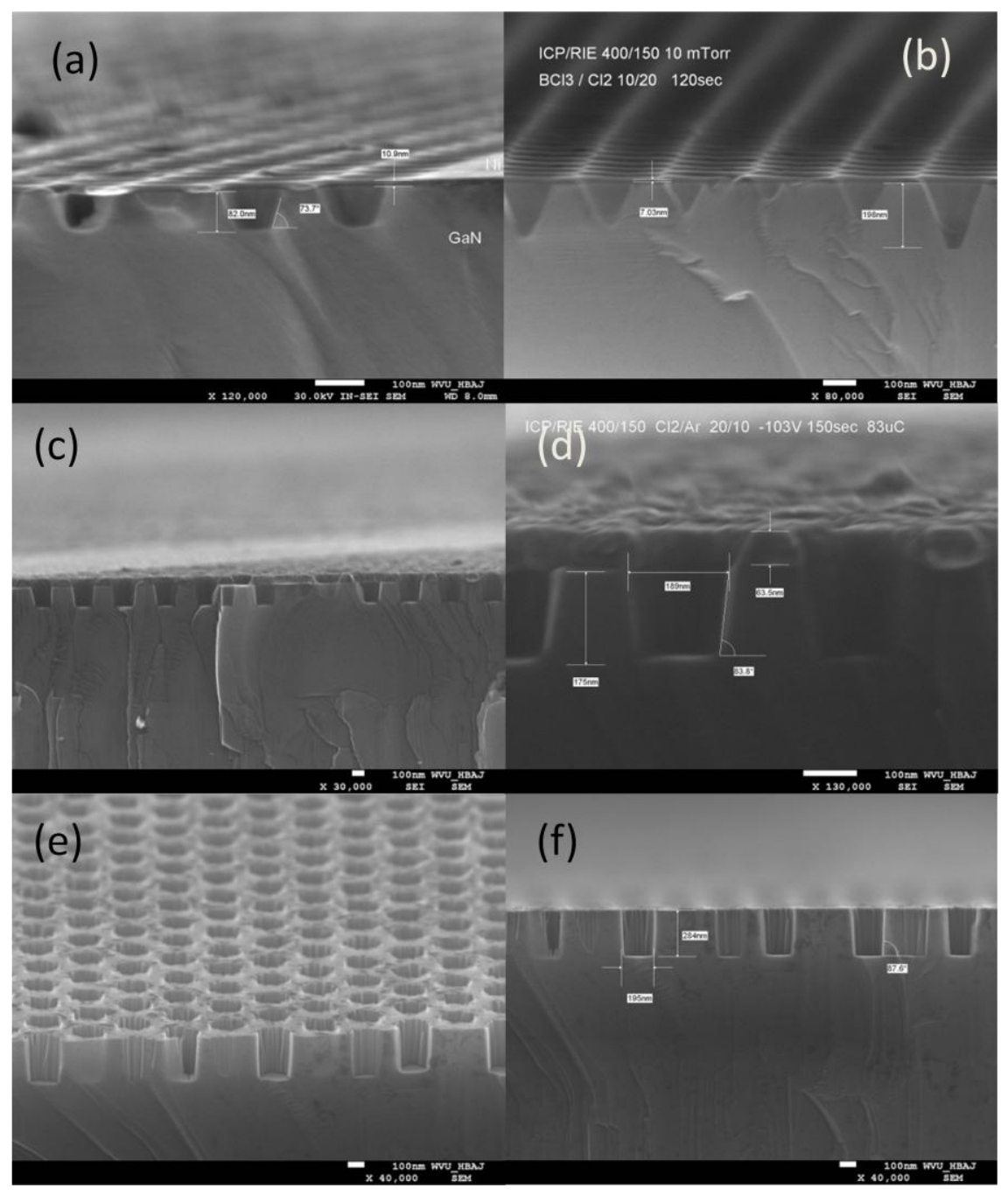

Figure 3-16: Cross-section SEM micrographs of Etching profiles of GaN structures: (a)-(b) Ni mask too thin for ICP etching process, (c)-(d) $\mathrm{PhC}$ etched with Ar based plasma, (e)-(f) $\mathrm{PhC}$ etched with $\mathrm{BCl} / \mathrm{Cl} 2$ based plasma.

\subsubsection{Fabrication of $\mathrm{PhC}$ on GaN using hard masks formed by two-step pattern transfer}

Figure 3-17 displays the flow diagram for this fabrication technique using a hard mask formed by a modified two-step pattern transfer process. The difference from the previously developed two-step etch back pattern transfer is that the Ni mask is formed by lift off instead of etch back. So the modified process combines two pattern transfers by lift-off and etch-back. This process is basically the same as etch-back fabrication method (described in 3.3.2) but it uses a negative resist instead of PMMA, in order to avoid the problem of PMMA reflowing. GaN substrate was cleaned and prebaked. $\mathrm{A} \mathrm{SiO}_{2}$ layer with a thickness of $200 \mathrm{~nm}$ was deposited on GaN by E-beam Evaporation (process parameters are listed in Table 3-2). The thickness of this layer is chosen such as to enable the final deep etching step in GaN 
considering the $\mathrm{SiO}_{2} / \mathrm{GaN}$ selectivity. This oxide layer was annealed at $700{ }^{\circ} \mathrm{C}$ for $5 \mathrm{~min}$ in air. Thus, this $\mathrm{SiO}_{2}$ layer becomes hard enough to undergo plasma etching based on $\mathrm{BCL}_{3} / \mathrm{Cl}_{2}$ chemistry. Negative tone ma-N 2403 resist was spun onto the silicon substrate using a spinner that was set at 4500 rpm for $45 \mathrm{sec}(\sim 270 \mathrm{~nm})$. The deposited resist was pre-baked on a hot plate at $90{ }^{\circ} \mathrm{C}$ for $3 \mathrm{~min}$ and subsequently left to cool to room temperature. The resist-coated substrate was diced into square pieces being ready to be exposed using the EBL system (JEOL 7600). PhC structures were defined by EBL with parameters: (1) accelerating voltage of $30 \mathrm{kV}$, (2) field size of $100 \mu \mathrm{m}$, (3) probe current $40 \mathrm{pA}$, and (4) ebeam dose of $70 \mu \mathrm{C} / \mathrm{cm}^{2}$. PhC patterns contained features with lattice constant $\mathrm{a}=333 \mathrm{~nm}$ and radius $=$ 100nm. An area of $2 \mathrm{~mm}$ x $100 \mu \mathrm{m}$ was covered placing PhC patterns, one after each other horizontally. The development process of the exposed resist was realized in ma-D 525 solution for $70 \mathrm{sec}$, and the process was stopped by rinsing in DI water for $1 \mathrm{~min}$. The samples were gently dried with a nitrogen gun. Developed samples were deposited $\sim 30 \mathrm{~nm} \mathrm{Ni}$ by e-beam evaporation (deposition parameters are described in Table 3-2). Once the samples were deposited $\mathrm{Ni}$, these were dip into acetone for lift-off for $30 \mathrm{~min}$. The $\mathrm{PhC}$ patterns on Ni layer were transferred into the $\mathrm{SiO}_{2}$ layer by $\mathrm{ICP}$ etching with $\mathrm{CF}_{4} / \mathrm{O}_{2}$ based plasma (dry etching parameters are listed in Table 3-3). The final dry etching step was done for 2 min in $\mathrm{BCL}_{3} / \mathrm{Cl}_{2}$ plasma with gas rates of $27 / 3(\mathrm{sccm}), 10 \mathrm{mTorr}$ pressure, $400 \mathrm{~W}$ ICP power and $150 \mathrm{~W}$ RIE power. This step transferred the air holes through the $\mathrm{SiO}_{2}$ into the $\mathrm{GaN}$ substrate. Once the dry etching was complete the remains of the surface mask were removed using a HF bath for 30sec. Finally, samples were cleaved in the middle of the PhC pattern, and then a thin film of Pt was deposited by sputtering for SEM imaging (cross section study).
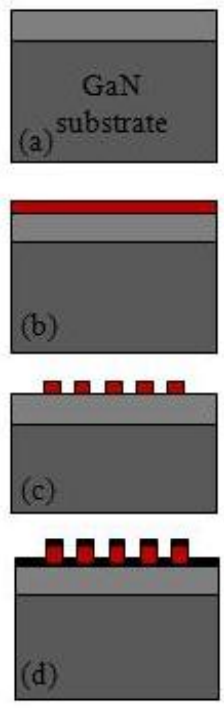

$\square$ Resist

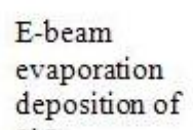

$\mathrm{SiO}_{2}$

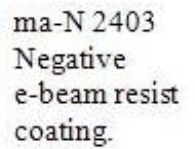

coating.

E-beam lithography patterning.

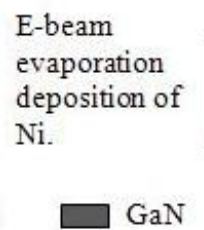

Metal lift-off.
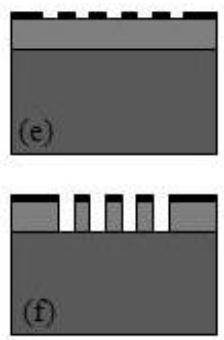

ICP etching of

$\mathrm{SiO}_{2}$.

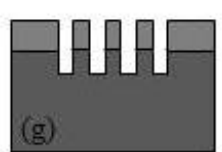

ICP etching

of $\mathrm{GaN}$.

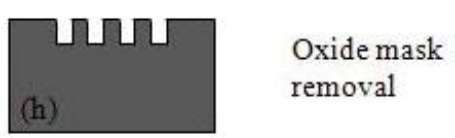

Figure 3-17: Process flow for the fabrication of $\mathrm{PhC}$ on $\mathrm{GaN}$ (lift-off and bilayer hard mask). 
Figure 3-18 shows all the fabrication stages with this technique; (a) top view of $\mathrm{PhC}$ pattern on ma-N 2403; (b) top view of PhC on Ni metal mask after lift-off process; (c) cross section of structure after ICP etching of $\mathrm{SiO}_{2}$ using $\mathrm{CF}_{4} / \mathrm{O}_{2}$ plasma; (d) cross section of $\mathrm{PhC}$ structures after ICP etching of $\mathrm{GaN}$ using $\mathrm{Cl}_{2} / \mathrm{BCl}_{3}$ plasma. (e) and (f) final structures on $\mathrm{GaN}$ after $\mathrm{SiO}_{2}$ removal.

Clearly, this second technique is capable of fabricating better GaN PhC structures with uniform 100 $\mathrm{nm} \mathrm{PhC}$ patterns, vertical airhole sidewalls, and a decent depth. As it can be seen in the SEM pictures, the $\mathrm{SiO}_{2}$ layer is almost intact after ICP etching of GaN, suggesting that the airholes can be etched deeper into the substrate. Simple lift-off process using only $\mathrm{Ni}$ as the hard mask also produces good $\mathrm{PhC}$ structures. However, the depth of the airholes is limited because Ni mask thickness. To fabricate thicker GaN PhC structures, a thicker Ni mask is needed, but the thickness of $\mathrm{Ni}$ is limited to $\sim 1 / 3$ of the thickness of the e-beam resist used.

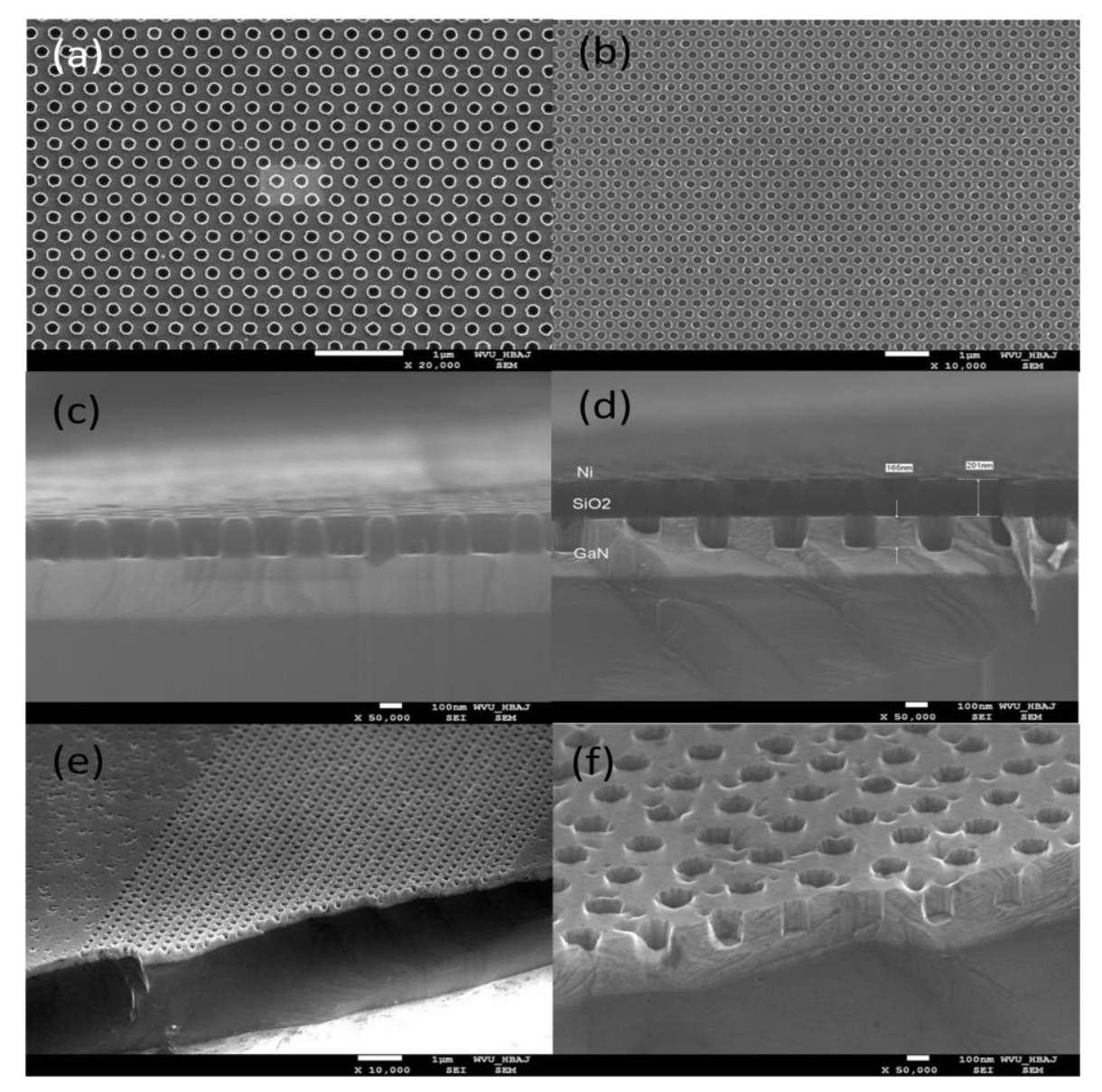

Figure 3-18: SEM micrographs of the steps for the $\mathrm{PhC}$ fabrication on GaN (lift off, experiment B): (a) $\mathrm{PhC}$ on resist maN2403, (b) Ni mask after lift-off process, (c) ICP etching of $\mathrm{SiO}_{2}$, (d) ICP etching of GaN, (f)-(g) $45^{\circ}$ view of PhC on Gan after mask removal. 
Hole Diameter

\begin{tabular}{cccc} 
Material & Design $[\mathrm{nm}]$ & Measured $[\mathrm{nm}]$ & Error percentage [\%] \\
\hline GaN & 200 & 195 & 2.5
\end{tabular}

The table above shows final measurements of the holes in the $\mathrm{PhC}$ structure on $\mathrm{GaN}$, and the computations of its respective error compared with the dimensions of the CAD design file.

\subsection{FABRICATION OF PHC STRUCTURES ON ITO}

Transparent conductors have always been of great interest for the research community or industry in general. Indium Tin Oxide (ITO) is the most widely used transparent conducting oxide due to its high electrical conductance and high optical transmittance. ITO is commonly used as ohmic and schottky contacts in many conventional optoelectronic and photonic devices as well as biosensing platforms. ${ }^{51-53}$ For example, ITO may be deposited atop the $\mathrm{GaN} \mathrm{PhC} \mathrm{structure} \mathrm{in} \mathrm{the} \mathrm{current} \mathrm{design} \mathrm{as} \mathrm{an} \mathrm{electrode} \mathrm{so}$ electric field can be applied to drive electrolytes in the microfluidic channel and nanocavity. The final part of the chapter is dedicated to the fabrication of ITO PhC structures. The dry etching of ITO in $\mathrm{BCL}_{3} / \mathrm{Cl}_{2}$-based plasma is first optimized to achieve fast etch rates and smooth etched surfaces. Like the case of $\mathrm{Si} \mathrm{PhC}$ fabrication, $\mathrm{Ni}$ is used as the etching mask, which is patterned by EBL with a negative resist and the lift off process.

For this experiment ITO film (260nm) was deposited on a Si (100 orientation) substrate using IBAD with $\mathrm{In}_{2} \mathrm{O}_{3} / \mathrm{SnO}_{2}(90 / 10 \mathrm{wt} \%$ ) source material (deposition parameters listed in Table 3-2). The as-deposit wafer was cut into pieces and partially mask with photo resist AZ4400 for dry etching study. Such study has been performed in Trion Minilock-Phantom III RIE system with fully integrated vacuum load-lock and an ICP source operating at $13.56 \mathrm{MHz}$. The samples were mounted using a thermal conductive paste on a Si wafer which was held by an electrostatic chuck and was Helium backside cooled.

$\mathrm{BCl}_{3} / \mathrm{Cl}_{2}$ is a potentially plasma chemistry for ITO dry etching. $\mathrm{BCl}_{3}, \mathrm{Cl}_{2}$ mixture offered higher etching rates and smooth morphology. However, $\mathrm{BCl}_{3} / \mathrm{Cl}_{2} / \mathrm{CF}_{4}$ mixture improves the dry etching process with higher etching rates and more smooth surfaces. In order to study the way how different process parameters influence the ITO etching, ICP power (100W to $600 \mathrm{~W}$ ), RIE power (50W to $250 \mathrm{~W}$ ), pressure (10mTorr to 60mTorr) and gas flow rate were varied according to the plots shown in Figure 3-19. Etch rates were computed from the depths measured by an alpha step profilometer.

Figure 3-19 (a) shows the etch rate of as-deposited ITO at 400W ICP power, $100 \mathrm{~W}$ RIE power, and 20 mTorr pressure, as a function of $\mathrm{Cl}_{2}$ concentration in a $\mathrm{BCl}_{3} / \mathrm{Cl}_{2}$ mixture with a fixed total flow rate of $24 \mathrm{sccm}$. As the $\%$ of $\mathrm{Cl}_{2}$ is increased, the etch rate initially increases due to more available reactive $\mathrm{Cl}$ 
radicals in the plasma. The etch rate peaks at $171 \mathrm{~nm} / \mathrm{min}$ when the $\% \mathrm{of}_{2}$ is equal to $20 \%$, and then decreases as the $\mathrm{Cl}_{2}$ concentration is further raised. The fact that the etch rate reaches the maximum at a low $\%$ of $\mathrm{Cl}_{2}$ reflects the dominance of pure physical bombardment in the etching process. $\mathrm{InCl}_{x}$ and $\mathrm{SnCl}_{x}$ have low volatilities at room temperature, and should result in slow etch rates. However, high ITO etch rates are obtained even at small percentages of reactive $\mathrm{Cl}_{2}$ due to efficient sputter desorption of the etch products in high fluxes of heavy $\mathrm{BCl}_{3}$ ions.
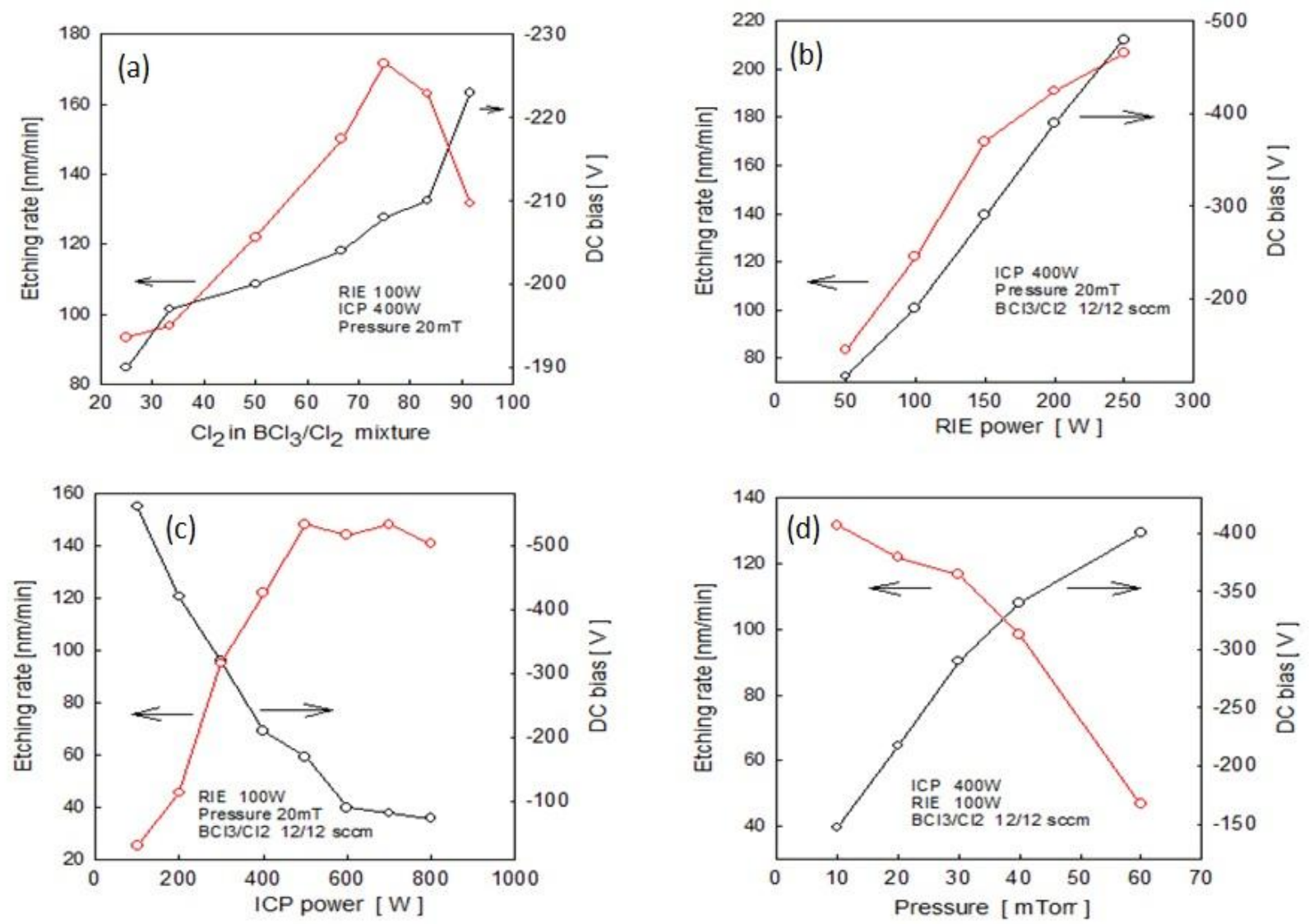

Figure 3-19: Etching rates of ITO based on $\mathrm{BCl}_{3} / \mathrm{Cl}_{2}$ plasma: (a) variation of percentage of $\mathrm{Cl} 2$ in a mix of $\mathrm{BCl} 3 / \mathrm{Cl} 2$; (b) variation of RIE power; (c) variation of ICP power; (d) variation of chamber pressure.

As seen in Figure 3-19 (a), the de self-bias decreases monotonically with increasing $\%$ of $\mathrm{Cl}_{2}$. Therefore, the reduced etch rates at high $\%$ of $\mathrm{Cl}_{2}$ are attributed to lower $\mathrm{BCl}_{3}$ ion concentrations as well as lower ion energies. The influence of the RIE power, ICP source power, and process pressure on the etch rate of ITO is displayed in Figure 3-19 (b), (c), (d) respectively. Also shown in these plots are the 
changes in dc self bias. As seen in Figure 3-19(b), as the RIE power is varied from 50 to $250 \mathrm{~W}$ while other parameters are kept constant, $400 \mathrm{~W}$ source power, 20 mTorr pressure, and 12/12 $\mathrm{sccm} \mathrm{BCl}_{3} / \mathrm{Cl}_{2}$ gas flow, the etch rate increases almost linearly from 83 to $207 \mathrm{~nm} / \mathrm{min}$. This can mainly be attributed to the increased dc bias or ion energy since higher-energy ions break bonds of ITO more efficiently and improve sputter desorption of nonvolatile etch products from the surface.

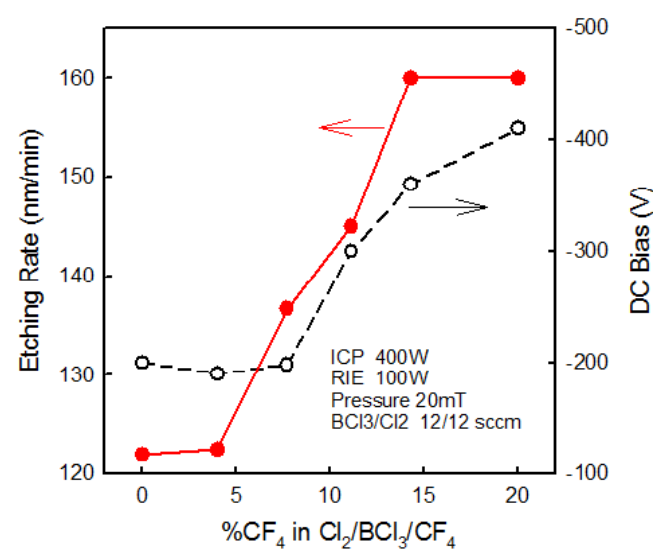

Figure 3-20: Etching rate of ITO when adding small percentages of $\mathrm{CF}_{4}$ to the $\mathrm{BCL}_{3} / \mathrm{Cl}_{2}$ mix.

In Figure 3-19 (c), ITO etch rate is shown as a function of ICP source power while the RIE power, pressure, and gas flow are held constant. As the source power is increased from 100 to $500 \mathrm{~W}$, the etch rate increases due to higher concentrations of reactive species and energetic ions. As the source power is raised further, the etch rate stabilizes and even decreases. Under these conditions, saturation of reactive species at the surface may occur, whereas the dc bias decreases due to increased plasma conductivity. As seen in Figure 3-19 (d), the etch rate decreases monotonically as the process pressure is increased from 10 to $60 \mathrm{mTorr}$ even though the dc bias increases and is nearly tripled at $60 \mathrm{mTorr}$. This is because more frequent collision at high pressures leads to a reduced electron density and thus less reactive $\mathrm{Cl}$. The chemical etching component goes down faster than the physical component rises due to higher dc bias, leading to reduced etch rates. ITO etching can be further enhanced by adding a small amount of CF4 into the $\mathrm{BCl}_{3} / \mathrm{Cl}_{2}$ mixture, as depicted in Figure 3-20. The addition of $\mathrm{CF}_{4}$ by more than $4 \%$ leads to a significant increase in the etch rate and resulted in smoother etched surfaces. The fastest etch rate is observed at $14 \% \mathrm{CF}_{4}$. The etch rate stabilizes as the $\%$ of $\mathrm{CF}_{4}$ is increased further. These results can be explained as follows. The ITO etching can be divided into two consecutive steps: (i) reduction in ITO to metallic In and $\mathrm{Sn}$ and (ii) removal of In and $\mathrm{Sn}$. In $\mathrm{BCl}_{3} / \mathrm{Cl}_{2}$ plasma, the first step, i.e., breaking of metaloxygen bonds, is carried out through physical ion bombardment. By contrast, in a $\mathrm{BCl}_{3} / \mathrm{Cl}_{2} / \mathrm{CF}_{4}$ plasma, reactive $\mathrm{C}$ from the $\mathrm{CF}_{4}$ gas may effectively reduce ITO into In and $\mathrm{Sn}$ through formation of $\mathrm{CO}_{2}$ and $\mathrm{CO}$. The reduction reactions may become much more efficient under ion bombardment. The In and Sn are 
then removed from the surface in the form of chloride compounds through sputter desorption. Therefore, the improved ITO etching in $\mathrm{BCl}_{3} / \mathrm{Cl}_{2} / \mathrm{CF}_{4}$ plasmas breaks efficiently the metal-oxygen bonds in ITO by $\mathrm{CF}_{4}$. However, excess $\mathrm{CF}_{4}$ may lead to the deposition of carbonaceous residues, slowing down the etching process. Once that ITO dry etching has been understood and optimized, the fabrication of ITO PhC structures can be realized. Figure 3-21 show the flow diagram for nanofabrication of these ITO structures using lift-off technique.

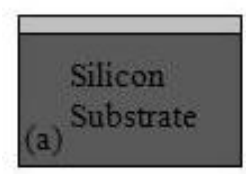

E-beam evaporation deposition of ITO

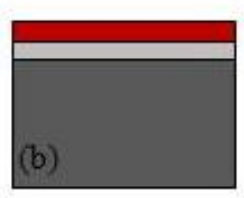

ma-N 2403

Negative e-beam resist coating.

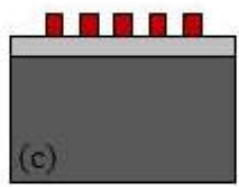

E-beam lithography patteming.

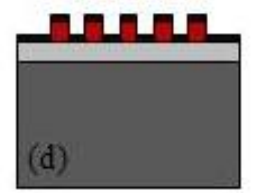

E-beam evaporation deposition of $\mathrm{Ni}$.
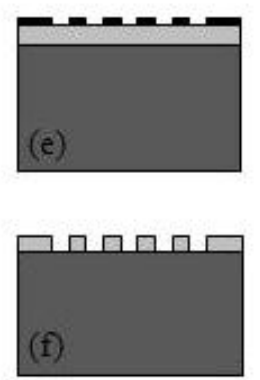

ICP etching of ITO and metal mask removing.

Figure 3-21: Process flow for the fabrication of PhC on ITO

Samples with as-deposited ITO film (260nm) were annealed at $500{ }^{\circ} \mathrm{C}$ in a $\mathrm{N}_{2}$ ambient for 5 min. After samples cooled down, negative tone ma-N 2403 resist was spun onto the ITO film using a spinner $4500 \mathrm{rpm}$ for $45 \mathrm{sec}(\sim 270 \mathrm{~nm})$. The deposited resist was pre-baked on a hot plate at $90{ }^{\circ} \mathrm{C}$ for $3 \mathrm{~min}$ and subsequently left cooling to room temperature. The resist-coated substrate was diced into two square pieces being ready to be exposed using the EBL system (JEOL 7600). PhC structures were defined by EBL with parameters: (1) accelerating voltage of $30 \mathrm{kV}$, (2) field size of $100 \mu \mathrm{m}$, (3) probe current $40 \mathrm{pA}$, and (4) e-beam dose of $75 \mu \mathrm{C} / \mathrm{cm}^{2}$. PhC patterns contained features with lattice constant a $=333 \mathrm{~nm}$ and radius $=100 \mathrm{~nm}$. An area of $2 \mathrm{~mm} \times 100 \mu \mathrm{m}$ was covered placing PhC patterns, one after each other horizontally. The development process of the exposed resist was realized in ma-D 525 solution for $70 \mathrm{sec}$, and the process was stopped by rinsing in DI water for $1 \mathrm{~min}$. The samples were dried very gently with a nitrogen gun. Developed samples were deposited $\sim 95 \mathrm{~nm}$ Ni layer by e-beam evaporation. Deposition parameters are listed in Table 3-2. Once the samples were deposited $\mathrm{Ni}$, these were dip into acetone for lift-off for $30 \mathrm{~min}$. The pattern transfer into ITO substrate was performed in an ICP RIE system at 10 mTorr pressure, $400 \mathrm{~W}$ ICP power, 150W RIE power and a gas mixture of $\mathrm{BCl}_{3} / \mathrm{Cl}_{2} / \mathrm{CF}_{4}$ with gas flows of 15/12/3 sccm for $90 \mathrm{sec}$. Finally, samples were cleaved in the middle of the PhC structure, and then they 
were sputtered a thin film of Pt for SEM imaging (cross section study). Figure 3-22 show some SEM pictures of the final structures in ITO (a) Top-view of maN-2403 on top of ITO film; (b) PhC structure on ITO, $45^{\circ}$ view; (c) and (d) cross section of PhC on ITO with Ni mask on top.

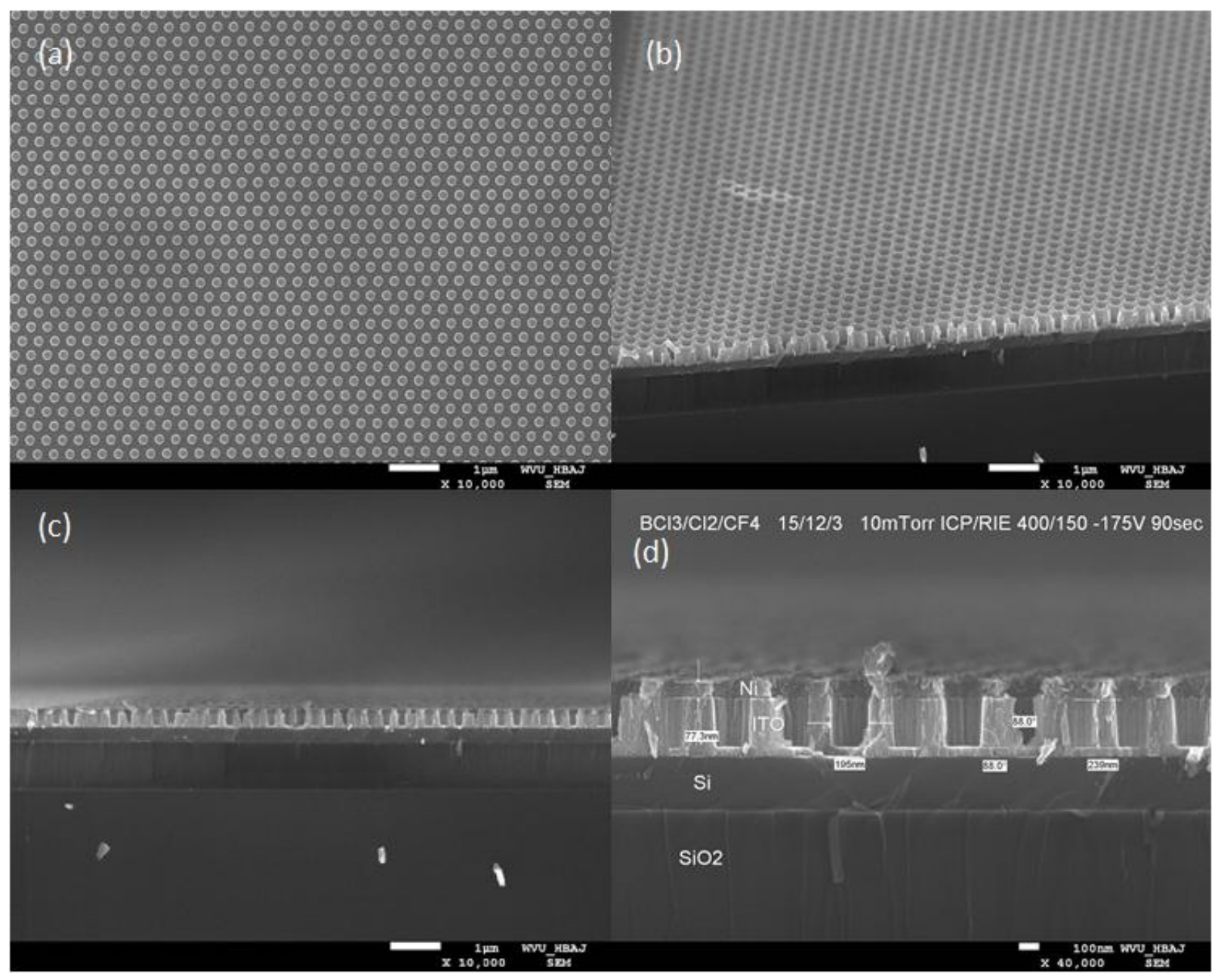

Figure 3-22: SEM micrographs of the fabrication of $\mathrm{PhC}$ structures on ITO film: (a) top view of $\mathrm{PhC}$ on resist ma-N 2403(75 $\left.\mu \mathrm{C} / \mathrm{cm}^{2}\right)$. (b) $45^{\circ}$-view of PhC on ITO, (c)-(d) cross-section of PhC structure on ITO.

\begin{tabular}{|cccc} 
& Hole Diameter & & \\
Material & Design $[\mathrm{nm}]$ & Measured $[\mathrm{nm}]$ & Error percentage [\%] \\
\hline ITO & 200 & 195 & 2.5 \\
\hline
\end{tabular}

The table above shows final measurements of the holes in the PhC structure on ITO, and the computations of its respective error compared with the dimensions of the CAD design file. 


\section{CHAPTER IV}

\section{SEALING OF PhC AIRHOLES BY GLANZING ANGLE DEPOSITION}

\subsection{INTRODUCTION}

Dielectric thin films are often deposited by means of e-beam evaporation deposition, during which the source material is heated until the material vaporizes and ejects atoms. Typically, this technique deposits atoms onto the substrate at a right angle respect to the substrate surface, as seen in Figure 4-1 (a). Evaporation deposition can also be done at an oblique-angle (also known as Glancing Angle Deposition GLAD) by placing a stationary substrate at non-normal angle with respect to the material vapor flux, as seen in Figure 4-1 (b). This kind of deposition was developed five decades ago and has been used to grow porous thin films ever since. Thin films deposited by GLAD have an inclined columnar morphology. These nanorod-like structures grow with an angle $(\beta)$ a little bit smaller than the angle of the vapor flux $(\alpha)$, as illustrated in Figure 4-1 (c).

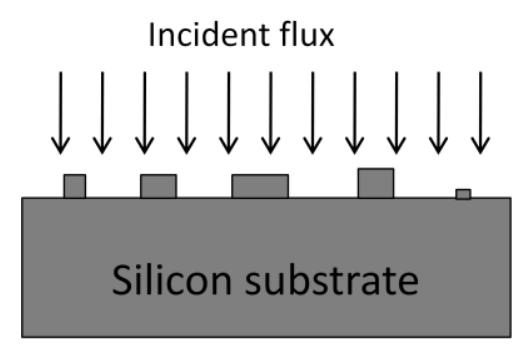

(a)

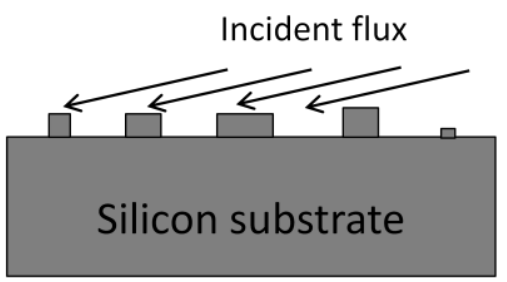

(b)

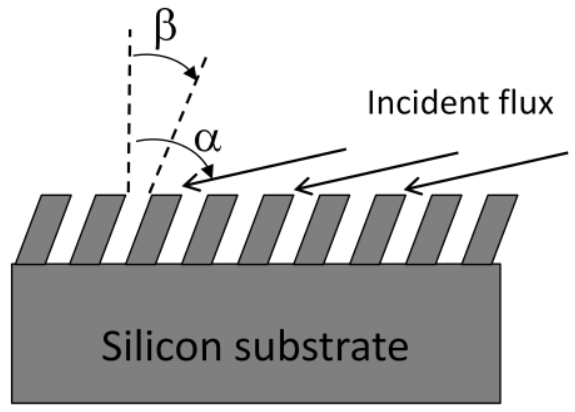

(c)

Figure 4-1: (a) Normal Deposition, (b) Oblique angle deposition, (c) Variables for Oblique deposition.

GLAD is a method based essentially on self shadowing and adatom diffusion properties of the material deposited at the atomic level. The material to be deposited can come from evaporating or sputtering a crucible or a target, respectively. ${ }^{54-56}$ Since the surface of a substrate is not perfectly flat, irregularities on the surface can prevent material flux from reaching such areas creating voids in the film. The adatom diffusion property of the evaporated material plays an important role because it can limit how far the atoms which reach the substrate diffuse on it. Instead of filling the voids caused by shadows, they 
form nuclei and islands (nucleation). Then under the limited condition of adatom diffusion, these islands enhance shadowing effects and allow the growth of tilted, randomly spaced columnar structures. ${ }^{57}$ It has been demonstrated the higher the angle $\alpha$, the greater the enhancement of the shadowing effects, and the further the columns are spaced from each other, ending up in a more porous thin film.

All the columnar structures have an inclination angle $\beta$ that is a strong function of the deposition angle $\alpha$. This inclination is said to be provoked by a possible short movement of the atoms on the top of the column in the direction of the incident flux of the material due to a conservation of parallel momentum that atoms have upon impact. ${ }^{57,58}$ The $\beta$ angle varies depending on the substrate temperature, deposition rates, vapor distribution and background pressure. Many studies have been conducted to comprehend the dynamics of $\beta$ angles. ${ }^{59}$ Figure 4-1 (c) illustrates these angles, and an empirical formula has come up providing the relationship between $\alpha$ and $\beta$ angles. This formula is known as "tangent rule" and it has proved very accurate for $\alpha<50^{\circ} .{ }^{60}$ However, it does not work for greater angles.

$$
\tan (\beta)=\frac{1}{2} * \tan (\alpha)
$$

Then the formula above has been changed by Tait et al. ${ }^{61}$ in order to predict $\beta$ for $\alpha>50^{\circ}$.

$$
\beta=\alpha-\sin ^{-1}\left(1-\frac{\cos (\alpha)}{2}\right)
$$

In this technique, as $\alpha$ increases $\beta$ also increases, and shadowing effects increase, forming larger voids in the film. This means that $\beta$ and porosity levels are directly coupled, and they cannot be independently managed. Thus column width or shape on these structures cannot be controlled. In order to overcome these issues a combination of GLAD and substrate rotation have been performed and dramatically changed the grown surface morphology. The final porous film consists of isolated, vertical, and evenly spaced columns. Figure 4-2 shows how columns can be tilted as a function of the deposition angle and rotation speed. 

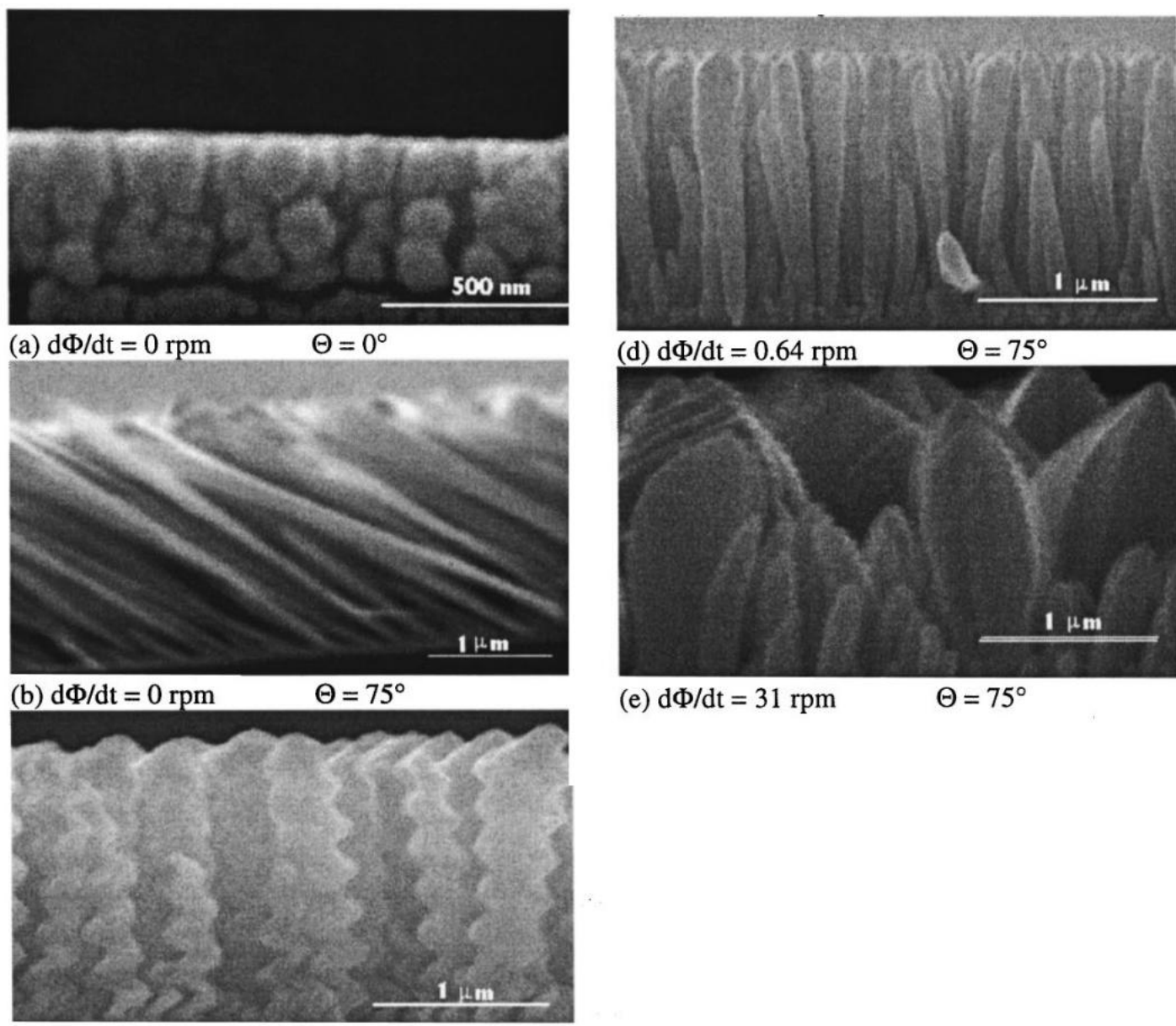

(e) $\mathrm{d} \Phi / \mathrm{dt}=31 \mathrm{rpm}$

$\Theta=75^{\circ}$

(c) $\mathrm{d} \Phi / \mathrm{dt}=0.16 \mathrm{rpm}$

$$
\Theta=75^{\circ}
$$

Figure 4-2: (a) deposition of film at right angle $\alpha$ and no substrate rotation, (b) deposition of film at $\alpha=75^{\circ}$ and no substrate rotation (tilted nanorod growing), (c) deposition of film at $\alpha=75^{\circ}$ and low substrate rotation (helical growing), (d) deposition of film at $\alpha=75^{\circ}$ and substrate rotation (vertical nanorod growing), (e) deposition of film at $\alpha=75^{\circ}$ and high substrate rotation (irregular surface morphology). ${ }^{62}$ 


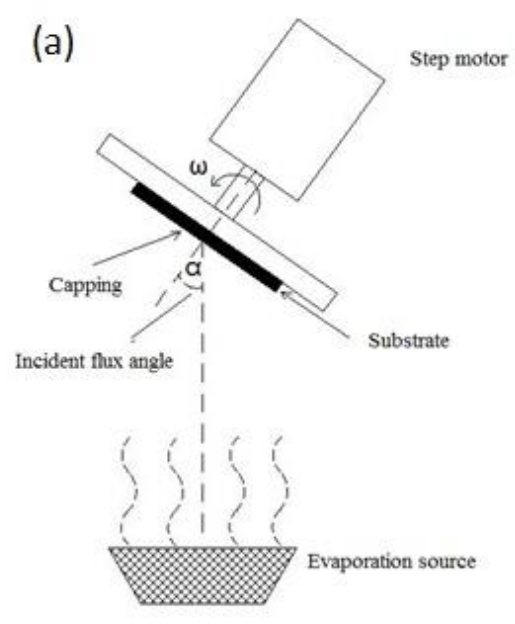

(b)

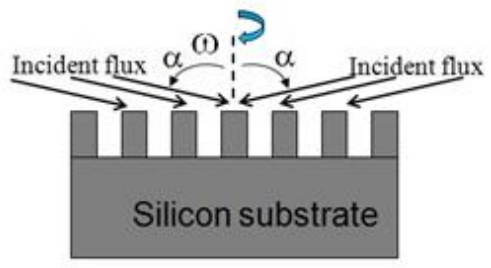

Figure 4-3: (a) GLAD schematic diagram, (b) material flux arriving onto the PhC structure by GLAD plus substrate rotation.

Figure 4-3 shows a schematic diagram for performing GLAD plus substrate rotation in an electron beam evaporation system. Note that, tilt angle and substrate rotation are not the only important parameters in this method. Pitch (deposition thickness per unit revolution $\mathrm{nm} / \mathrm{rev}$ ) also plays an important role in enabling the precise growth of many interesting sculptured films including C, S, zigzag, and helical shapes with a variety of metal, insulator or semiconductor materials. ${ }^{63}$ Figure $4-4$ shows all these types of engineered structures grown by varying $\alpha$, pitch, $\omega$, and material specific properties. However, the objective of our work is to grow a uniform thin film to seal nanoscale air holes and cap Si PhC structures. Therefore, a new deposition recipe must be developed to grow amorphous conformal films and avoid directional growth of nanorod-like structures.

a)

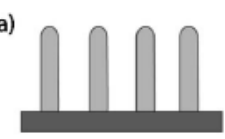

d)

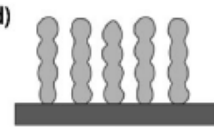

g)

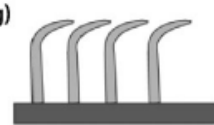

j)

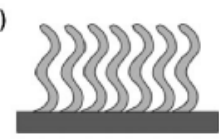

b)

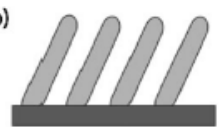

c)

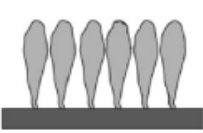

e)

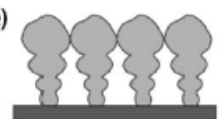

f)

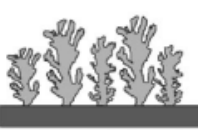

h)

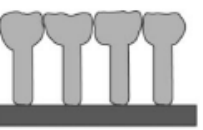

i)

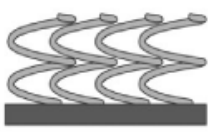

k)

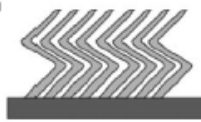

I)

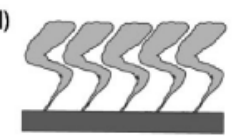

Figure 4-4: Various structures attained by GLAD plus rotation and deposition control. (a) Vertical pillars, (b) inclined pillars, (c),(d),(e) variable diameter pillars, (f) fractal like structures, (g),(h) columns with capping layers, (i) helix, (j) waved columns, (k) zig-zag, (l) zig-zag pillar with variable diameter. ${ }^{2}$ 


\subsection{GLAD SETUP}

In this work we modified our existing conventional e-beam evaporation system so as to carry out the GLAD study. In order to achieve high quality nano-engineered films with controllable geometries by mean of GLAD plus substrate rotation, many requirements should be met and are listed as follows: ${ }^{63}$

- Precise control over substrate rotation ( $\omega$ ) and tilting substrate angle ( $\alpha$ ) not only continuously but also discretely in time. This makes pc-controlled stepper motors perfect for this kind of duty.

- Deposition flux should be highly uniform and collimated, so that a very narrow angular distribution of the flux will reach the substrate.

- A high vacuum chamber (at least $10-^{8}$ Torr) is strongly recommended to avoid or at least minimize scattering effects because this can affect directionality in the vapor arriving onto the substrate.

- A complete control over the deposition conditions such as deposition rates and substrate temperatures.
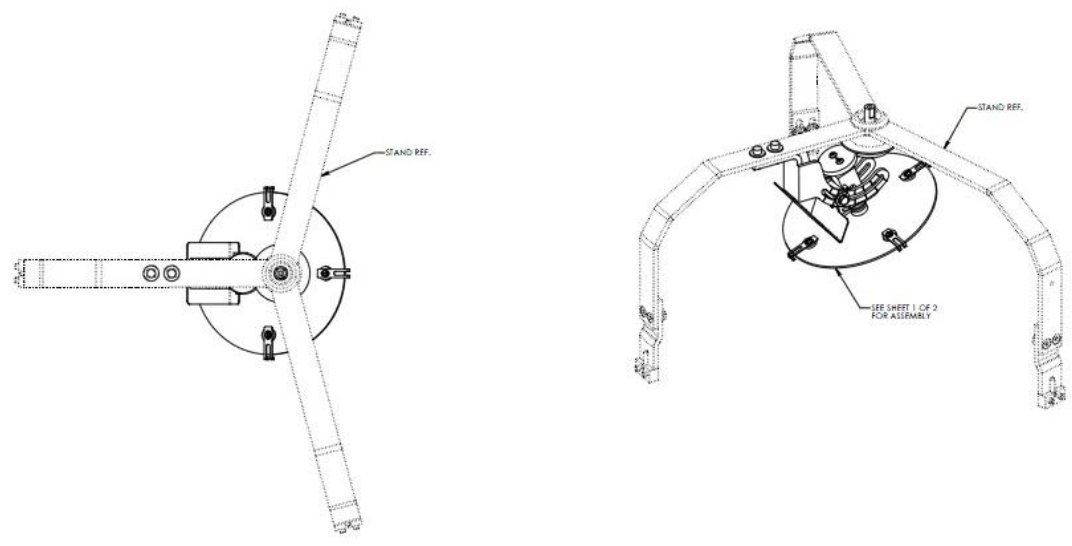

Figure 4-5: GLAD fixture from TEMESCAL for e-beam evaporator BJD 2000, also from TEMESCAL.
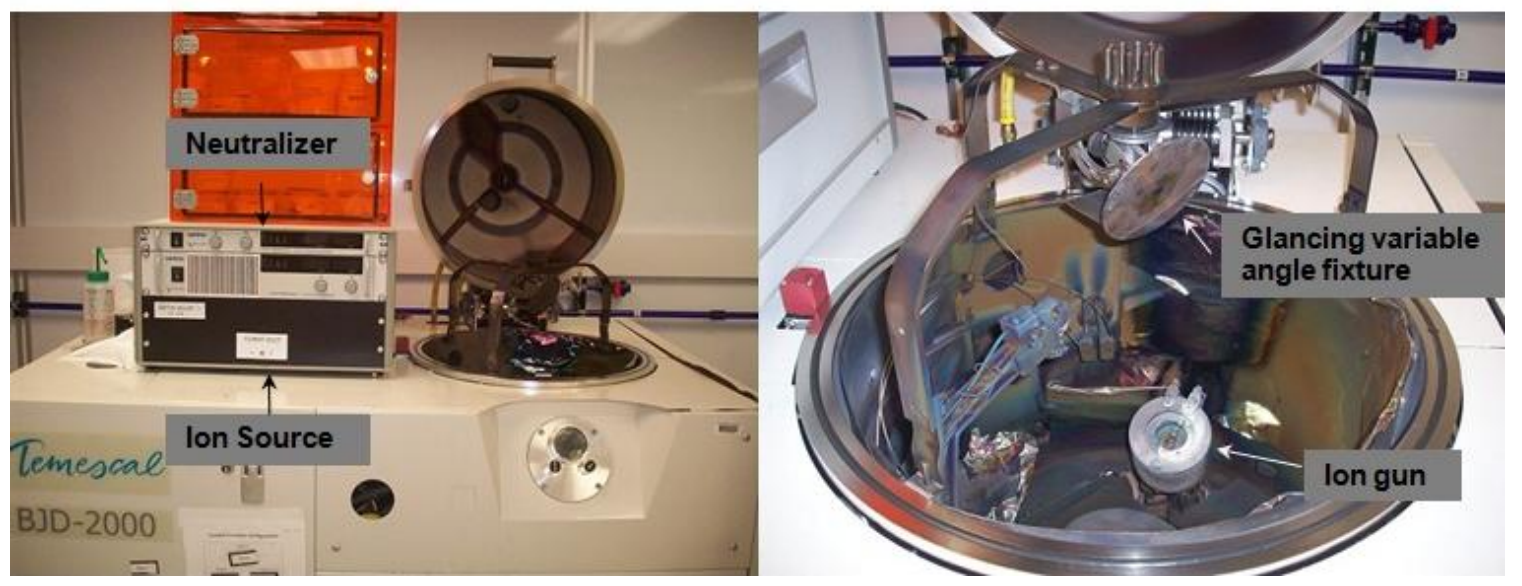

Figure 4-6 : (left) Electron beam evaporator, Ion beam assisted deposition equipment, and Glad fixture, (right) planetary was completely removed and replaced it with GLAD apparatus. 
A GLAD fixture was purchased from TEMESCAL. The apparatus has a 150mm single substrate holder, which can tilt from 0 to 90 degrees with a step resolution of 5 deg and manual angle variation. Figure 4-5 shows this mechanical device. Such a fixture was adapted onto the planetary substrate holder of the BJD2000 TEMESCAL e-beam evaporator. Also this system is equipped with a Neutralizer and Ion source both from Xantrex for Ion Beam Assisted deposition (IBAD) required for silicon oxide deposition. Figure 4-6 illustrates the set up in the e-beam evaporator and also shows all the necessary equipment for IBAD.

\subsection{OPTIMIZATION OF GLANCING ANGLE DEPOSITION OF $\mathrm{SiO}_{2}$}

$\mathrm{PhC}$ structures were fabricated on Si using electron beam lithography and plasma etching. The samples were mounted firmly onto the GLAD wafer holder which was rotated towards the source flux to fix the deposition angle. $\mathrm{SiO}_{2}$ film deposition was conducted with IBAD in an electron beam evaporation system. The oxygen flow was set at $20 \mathrm{sccm}$. The Ion source current was $1.3 \mathrm{Amps}$, and neutralizer current was 21 Amps. Before the deposition, the chamber was evacuated to a base pressure of 1E-5 Torr and the pressure stabilized at 1.5E-4 Torr during the deposition. The source to substrate distance was approximately $40 \mathrm{~cm}$. A crystal oscillator was used to monitor the thickness of the $\mathrm{SiO}_{2}$ film.

We have confirmed that GLAD with a constant angle resulted in nanorod-like structures, as shown in Figure 4-8 (a). To deposited uniform amorphous $\mathrm{SiO}_{2}$ films, the flux angle must be varied during the deposition process. The flux angle variation can be exponential or linear with time. It has been demonstrated by K. Robbie (3) that linearly-varied flux angle yielded cracks in the films due to stress effects. On the other hand, exponentially-varied angle release the stress of the films. Therefore, in this work, we exponentially varied the flux angle, i.e., $\alpha=A * e^{-B * t}$ in an effort to optimize the GLAD of $\mathrm{SiO}_{2}$ on top of the $\mathrm{Si} \mathrm{PhC}$ structures.

A set of three samples were prepared with $\mathrm{SiO}_{2}$ deposited with different values of $\mathrm{B}$ in the above flux angle expression. Table 4-1 summarizes all the key processing parameters for each sample. Figure 4-7 (b), (c), and (d) display two profiles that describe the flux angle variation in time: (i) the black line indicates the theoretical flux angle variation, and (ii) the red represents the real flux angles set on the GLAD wafer holder and used during the deposition. The discrete angles were selected with the smallest separation determined by the resolution of the GLAD fixture. The time on the X-axis scales with the thickness of the deposited films. And finally, it is important to mention that the deposition rates and substrate rotation were kept constant along the whole deposition process. 


\begin{tabular}{lllcc} 
& Material & $\begin{array}{c}\text { Theoretical deposition } \\
\text { variation of flux angle }\end{array}$ & Rotation speed & Deposition rate \\
\hline Sample b & $\mathrm{SiO}_{2}$ & $\alpha=110 * \mathrm{e}(-0.007 * \mathrm{t})$ & $6 \mathrm{RPM}$ & $2^{\circ} \mathrm{A} / \mathrm{sec}$ \\
\hline Sample c & $\mathrm{SiO}_{2}$ & $\alpha=130 * \mathrm{e}(-0.021 * \mathrm{t})$ & $6 \mathrm{RPM}$ & $2^{\circ} \mathrm{A} / \mathrm{sec}$ \\
\hline Sample d & $\mathrm{SiO}_{2}$ & $\alpha=150 * \mathrm{e}(-0.051 * \mathrm{t})$ & $6 \mathrm{RPM}$ & $0.5^{\circ} \mathrm{A} / \mathrm{sec}$
\end{tabular}

Table 4-1: Process parameters for GLAD deposition for optimization of capping layer
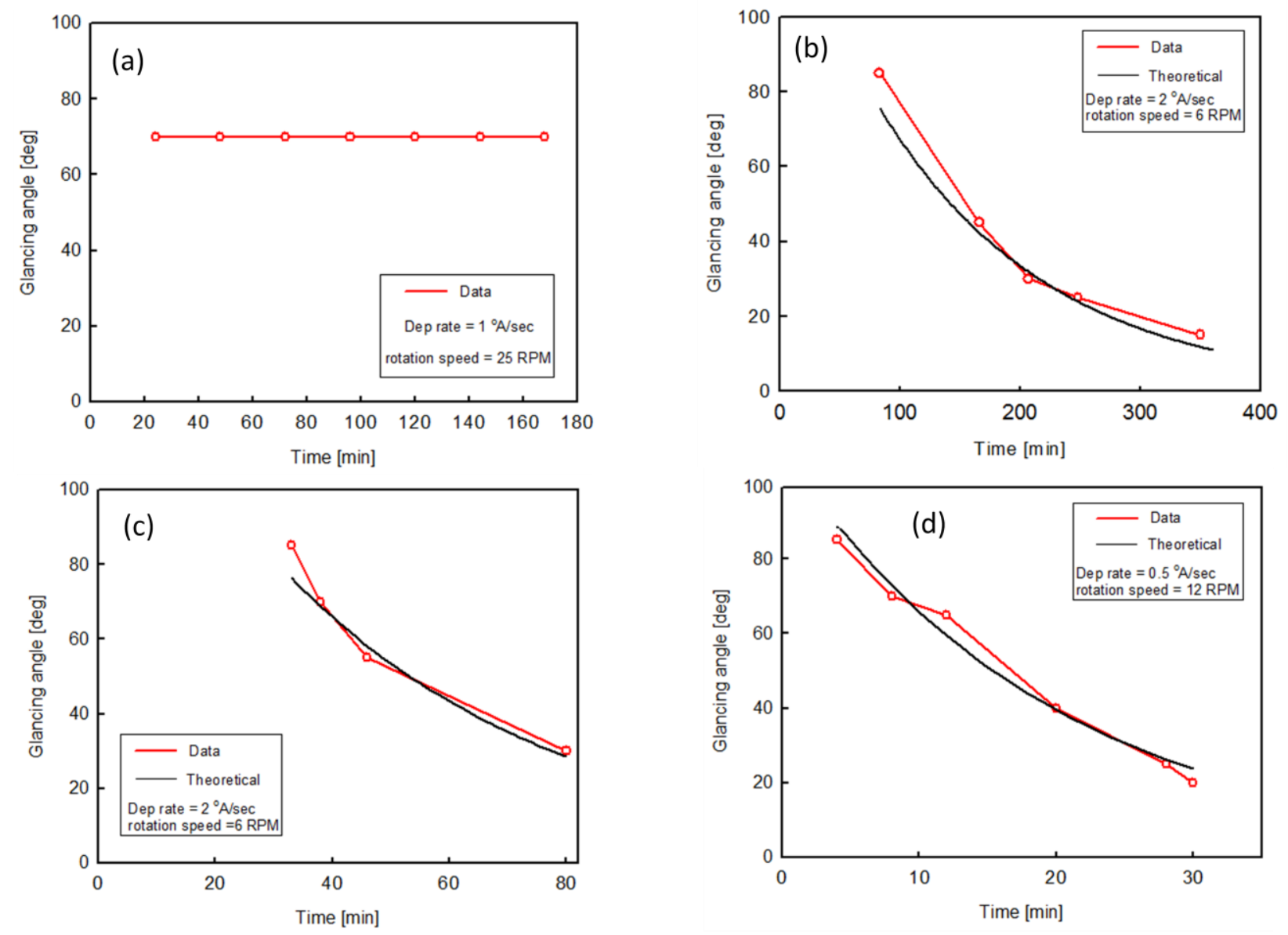

Figure 4-7: (a) Deposition at fixed angle $75^{\circ}$.Profiles of flux angle variation in time with different values of $\mathrm{B}$ in the exponential expression, (b) 0.007 , (c) 0.02 , (d) 0.05 .

It can be seen in Figure 4-8 (b), the lowest value of $B=0.007$ resulted in very long material deposition run giving rise to a very thick capping film $(\sim 2.5 \mu \mathrm{m})$ which completely seals the airholes. In Figure 4-8 (c), for a value of $B=0.021$, a much thinner film was obtained $(\sim 250 \mathrm{~nm})$. However, the film was too bumpy, and airholes are not completely covered. Figure 4-8 (d) depicts a film deposited with $\mathrm{B}=$ 0.05 on the exponential variation of the flux angle. The film is thin and smooth, but again, is does not completely cover the airhole. These results suggest that $\mathrm{SiO}_{2}$ capping layers can be deposited on top of $\mathrm{PhC}$ structures by GLAD with exponentially reduced flux angle, and complete sealing of the airholes 
requires a good control of the deposition time and flux angles. It is essential to change the flux angle gradually during the deposition.

It is also seen, that the more the number of variations of the flux angle, the smoother the final capping layer. This is an important fact because uniform sealing films are desirable in order to keep optical properties of the PhC structures unaltered.

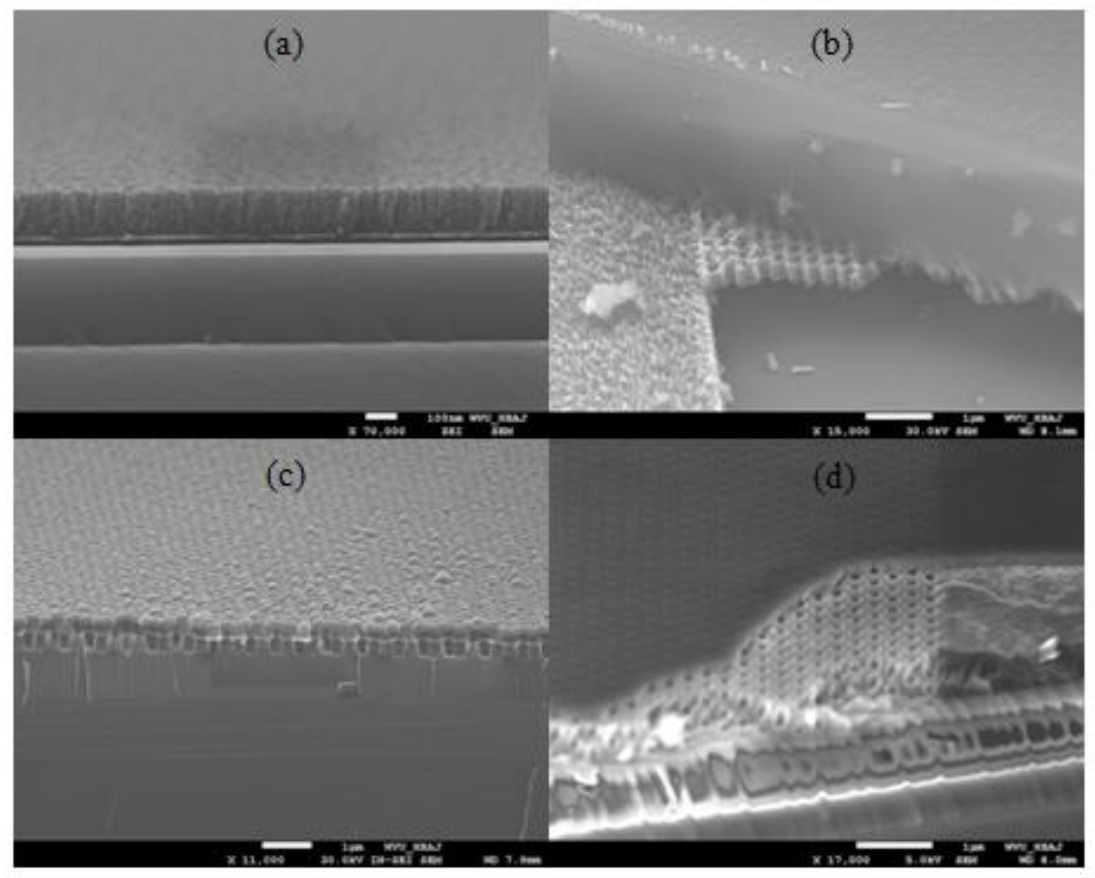

Figure 4-8: SEM micrographs of final capping layer for each profile proposed in Figure 4-7.

\subsection{PhC SEALING BY GLAD WITH SUBSTRATE ROTATION}

As it was explained before, in the proposed biosensor architecture, the PhC structure (air holes $\mathrm{a}=$ $290, r=80 \mathrm{~nm}$ ) will be integrated with a micro fluidic channel which will be interacting continuously with aqueous solutions. Consequently, The PhC air holes must be fully sealed to keep the phonic band gap constant and retain low levels of scattering losses.

This process underwent further optimization in order to reduce the final thickness of the oxide film and assure entirely sealed airholes. To accomplish such a structure, we came up with a recipe to obtain fully capped $\mathrm{PhC}$ structures and it can be divided into two processes. First, a deposition is performed a $\mathrm{t}$ a constant large flux angle. Under these conditions, nanopillars grow along the flux direction. Their tops spread around until they coalesce at a certain height where $\mathrm{PhC}$ holes are sealed. This material deposition is carried out at low substrate rotation and high pitch rate ( $\mathrm{nm} / \mathrm{rev}$ ). This causes the film to become highly porous and to seal airholes more quickly. Second, a final layer is deposited to reduce surface roughness of 
the film grown in the previous step. This layer was deposited using the exponential flux angle variation as explained above. Figure 4-9 shows all the parameters for the growth of such films. As it was mentioned before, this fixture is manual, so each data point in the plots represents a flux angle set on the GLAD apparatus.
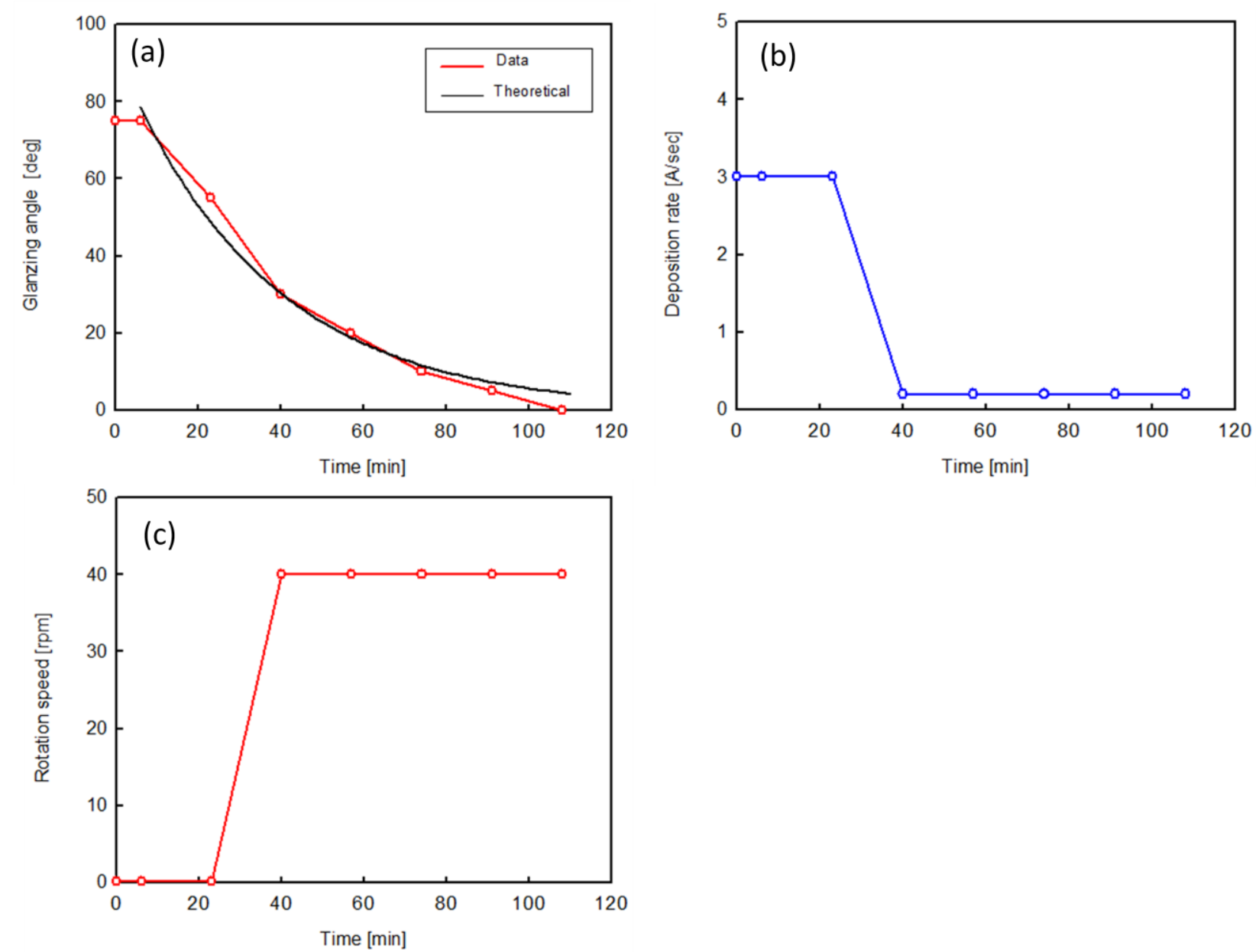

Figure 4-9: Profiles of the main deposition parameters for GLAD: (a) flux angle [ $\alpha=100 * \exp (-0.028 * t)]$, (b) deposition rate, (c) rotation speed of the substrate. $\mathrm{t}=20 \mathrm{~min}$ is the time when the exponential decay of the flux angle started.

For each run of deposition, the system was pumped to a background pressure of 1.0E-5 Torr. The chamber pressure during deposition stabilized at 1.5E-4 Torr. The Neutralizer and Ion source currents were set at 21 Amps and 1.3 Amps, respectively. Before loading, the PhC samples were transversally cleaved across the PhC direction. The cleaved samples were coated with a thin sputtered deposited Pt for the purpose of SEM images. The tooling factor used for regular deposition cannot be used to measure the deposition rates of GLAD. Hence, the thickness was approximated based on SEM measurements. Figure 4-10 shows the $\mathrm{PhC}$ structure fully capped with $\mathrm{SiO}_{2}$ grown using the parameter profiles indicated in Figure 4-9. Figure 4-10 (a) and (b) show the top views of the capping layer on top of the photonic crystal 
structure. (c) and (d) show the cross sections of the entire structure. As seen from these images, pillar growth occurred before the airholes were sealed. The coalescence of the nanopillars is seen at a thickness of $\sim 260 \mathrm{~nm}$. On top of the nanopillars, a much denser layer is seen, which reduces the roughness of the nanopillar layer. Also another important feature seen from the SEM images is that due to the initial growth at a large constant flux angle, very little material was deposited on the sidewalls of the airholes. This is important because any material extended into the airholes represents a perturbation to the photonic band gap structure.

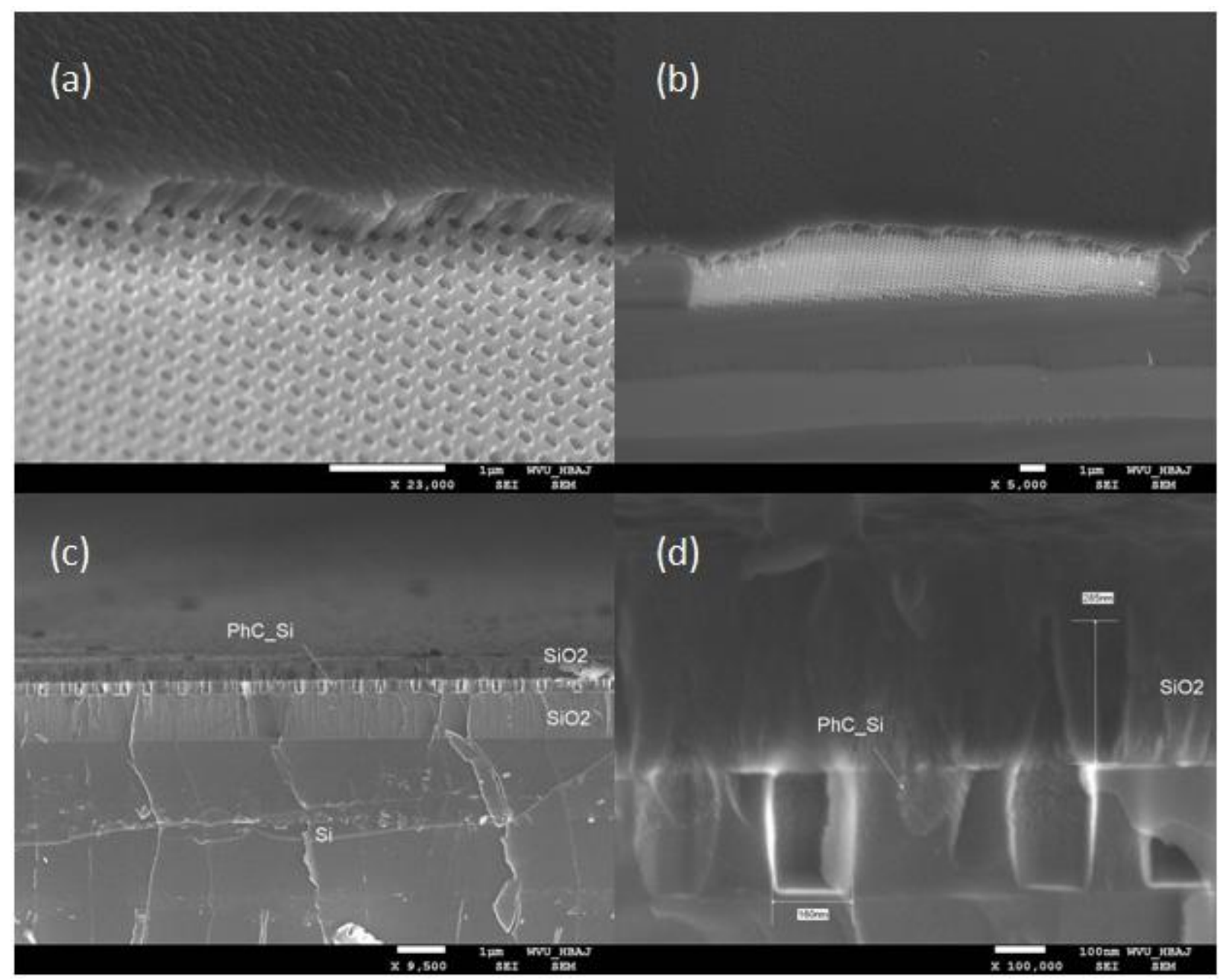

Figure 4-10: SEM micrographs of final capping layer on top of PhC structure (total thickness 320nm) 


\section{CHAPTER V}

\section{FABRICATION OF SUSPENDED PhC NANOCAVITY SLAB FOR BIOSENSING TESTING}

In this task, we fabricated suspended PhC nanocavity slabs on a Silicon-on-insulator (SOI) substrate. The structures will be used as the testing bed for measuring the optical confinement within the defect of the $\mathrm{PhC}$ structure using quantum dots. This experiment will allow us to experimentally determine the resonance enhancement factor before the final integration of $\mathrm{PhC}$ nanocavity resonators with microfluidic channels. The characterization results will be compared with our simulation results and utilized to adjust the dimensions of the $\mathrm{PhC}$ nanocavity structures.

\subsection{FABRICATION PROCESS}

Figure 5-1 shows the process flow that was carried out for fabricating a suspended PhC slab on $\mathrm{Si}$ using lift-off technique. A commercial SOI wafer was used, which had a $260 \mathrm{~nm}$ top Si (100) layer and a $1 \mu \mathrm{m}$ silicon dioxide buried underneath. The wafer was broken into small pieces, which were cleaned and dehydrated. E-beam resist ma-N 2403 was spin coated at $4500 \mathrm{rpm}$ for $45 \mathrm{sec}(\sim 270 \mathrm{~nm})$. The deposited resist was pre-baked on a hot plate at $90{ }^{\circ} \mathrm{C}$ for $3 \mathrm{~min}$ and subsequently left to cool to room temperature. The resist-coated samples were exposed using the EBL system (JEOL 7600). PhC structures were defined by EBL with parameters: (1) accelerating voltage of $30 \mathrm{kV}$, (2) field size of $100 \mu \mathrm{m}$, (3) probe current 40 $\mathrm{pA}$, and (4) e-beam dose of $70 \mu \mathrm{C} / \mathrm{cm}^{2}$. PhC patterns contained features with lattice constant $\mathrm{a}=260 \mathrm{~nm}$ and radius $=84 \mathrm{~nm}$. An area of $2 \mathrm{~mm}$ x $100 \mu \mathrm{m}$ was covered placing PhC patterns, one after each other horizontally. The development process of the exposed resist was realized in ma-D 525 solution for $70 \mathrm{sec}$, and the process was stopped by rinsing in DI water for $1 \mathrm{~min}$. The samples were dried very gently with a nitrogen gun. Developed samples were deposited $\sim 30 \mathrm{~nm}$ Ni using e-beam evaporation. Deposition parameters are listed in Table 3-2. Once the samples were deposited $\mathrm{Ni}$, these were dip into acetone to lift-off the Ni in the airhole areas so as to form a Ni mask with PhC patterns. The pattern transfer into Si substrate was carried out using an ICP RIE system in $\mathrm{CF}_{4} / \mathrm{O}_{2}$ based plasma with gas flow rates of $18 / 2$ sccm (standard cubic centimeter per minute), at a Pressure of $10 \mathrm{mTorr}$, ICP power of 400W, and RIE power of $150 \mathrm{~W}$ for $1 \mathrm{~min}$. This is sufficient to etch through the top Si layer. Wet etching was performed 
for $15 \mathrm{~min}$, on the samples with $\mathrm{Ni}$ etchant to remove the metal mask. Finally, the samples were merged in $\mathrm{HF}$ (hydrofluoric acid) for $30 \mathrm{sec}$ to remove the $\mathrm{SiO}_{2}$ layer underneath leading to the formation of a final suspended structure on Si substrate. Finally, samples were cleaved in the middle of the PhC pattern, and then coated with a thin sputter-deposited Pt for the purpose of SEM imaging (for cross section study). Figure 5-2 (a) and (b) show the suspended PhC nanocavity slab structure, whereas (c) and (d) are cross sectional view of the same structure.

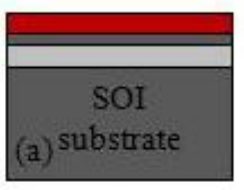

ma-N 2403

Negative e-beam

resist coating.

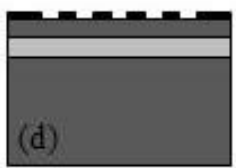

Metal lift-off.

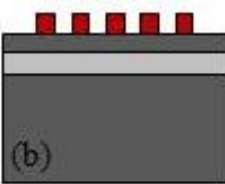

E-beam

lithography

patterning.

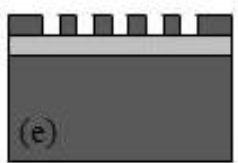

ICP etching of $\mathrm{Si}$ and metal mask removing.

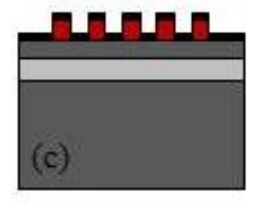

E-beam evaporation deposition of $\mathrm{Ni}$.

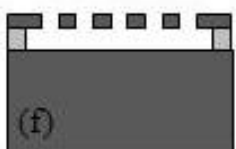

Wet etching oxide layer removing.

Resist

Silicon dioxide

Silicon

Nickel

Figure 5-1: Process flow for fabrication of Photonic crystal slab on Si 


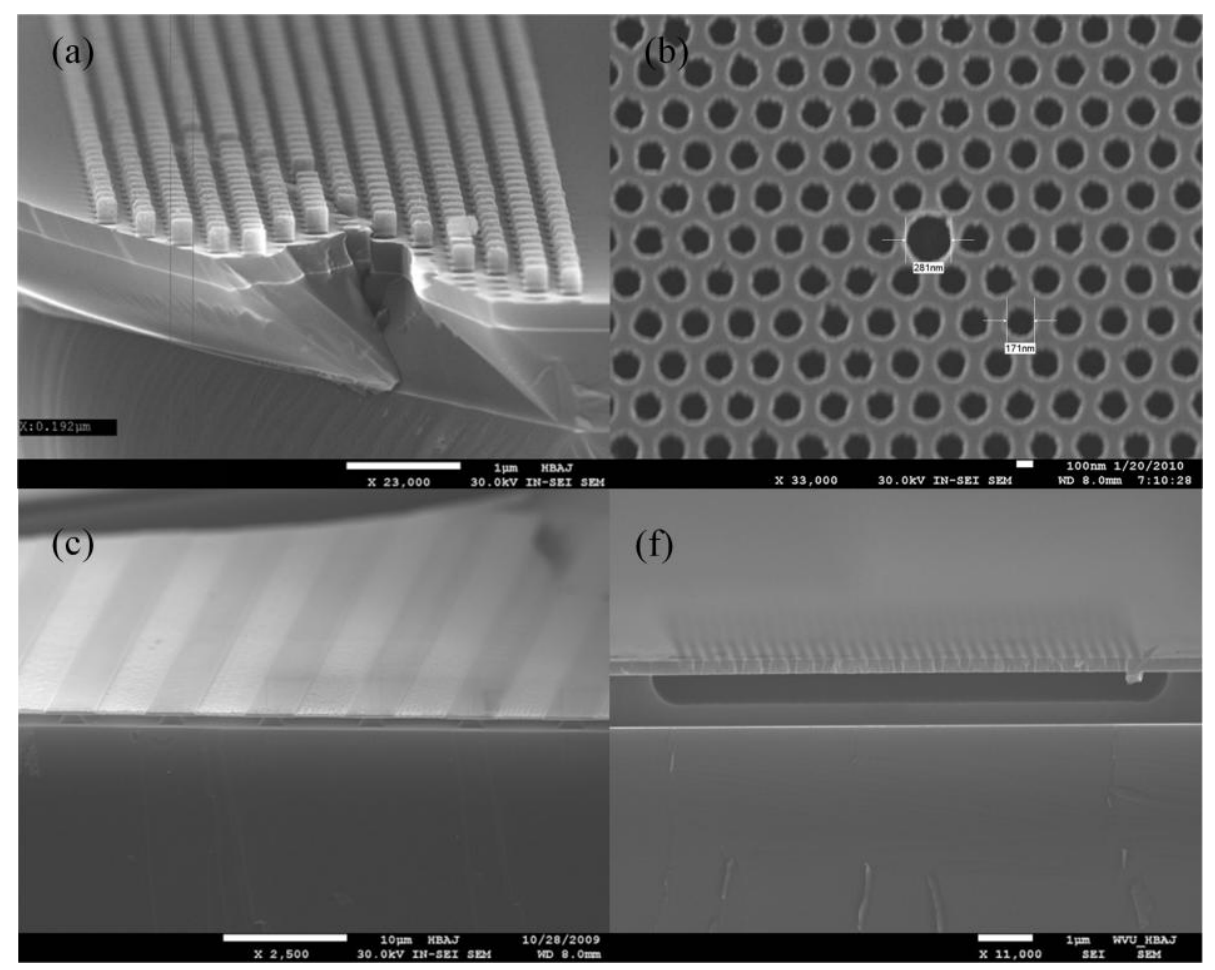

Figure 5-2: SEM micrographs of fabrication process of a PhC slab on Silicon: (a) cross-section of PhC on ma-N 2403 resist, (b) top-view of $\mathrm{PhC}$ slab on Si plus nanocavity defect, (c) $45^{\circ}$ view of many suspended PhC slabs on SOI, (d) crosssection of a final suspended $\mathrm{PhC}$ slab on SOI.

\subsection{FLUORESCENCE CHARACTERIZATION}

The fluorescence measurements will be carried out in a chemistry lab where a setup for testing these structures was built as seen in Figure 5-3. This experiment is designed to investigate optical confinement within the nanocavity defect inside the $\mathrm{PhC}$ slab on $\mathrm{Si}$. The tentative procedure is briefly described as follows. A droplet of a solution of toluene with quantum dots (EviDot 1100 core comprised of $\mathrm{PbS}$ nanocrystals, with emission and excitation wavelengths of $1100 \mathrm{~nm}$ and $632 \mathrm{~nm}$ respectively) is placed in the middle of a square piece of glass. Immediately, the sample with the $\mathrm{PhC}$ slab is pressed upside down on top of the quantum dot solution on the glass (PhC slab faced the glass surface). The piece of glass is placed onto a sample holder of a fluorescent microscope. This microscope is externally equipped with a laser source (638 nm red) and an infrared camera. The laser source pumps light with a wavelength of 638 nm into a dichroic mirror which bent the light beam into the sample to excite the quantum dots inside the $\mathrm{PhC}$ structure. The $\mathrm{PhC}$ structure will be designed to have a photonic bandgap between $994 \mathrm{~nm}$ to $1224 \mathrm{~nm}$ (values extracted from simulation on MPB) and its nanocavity defect will be designed to allow a single mode at $1100 \mathrm{~nm}$. Therefore, when quantum dots are excited, they start emitting light at a wavelength of $1100 \mathrm{~nm}$. All this emission is prohibited in the $\mathrm{PhC}$ structure except in the lattice 
nanocavity defect of the $\mathrm{PhC}$ structure. Therefore this nanocavity confines all the quantum dot emission. This light coming out from the nanocavity is directed to a mirror through the dichroic mirror (this mirror was also a filter that let only $1100 \mathrm{~nm}$ or higher frequencies through). The second plain mirror bends the light toward a view of an IR camera for optical analysis on the surface of the PhC slab structure.

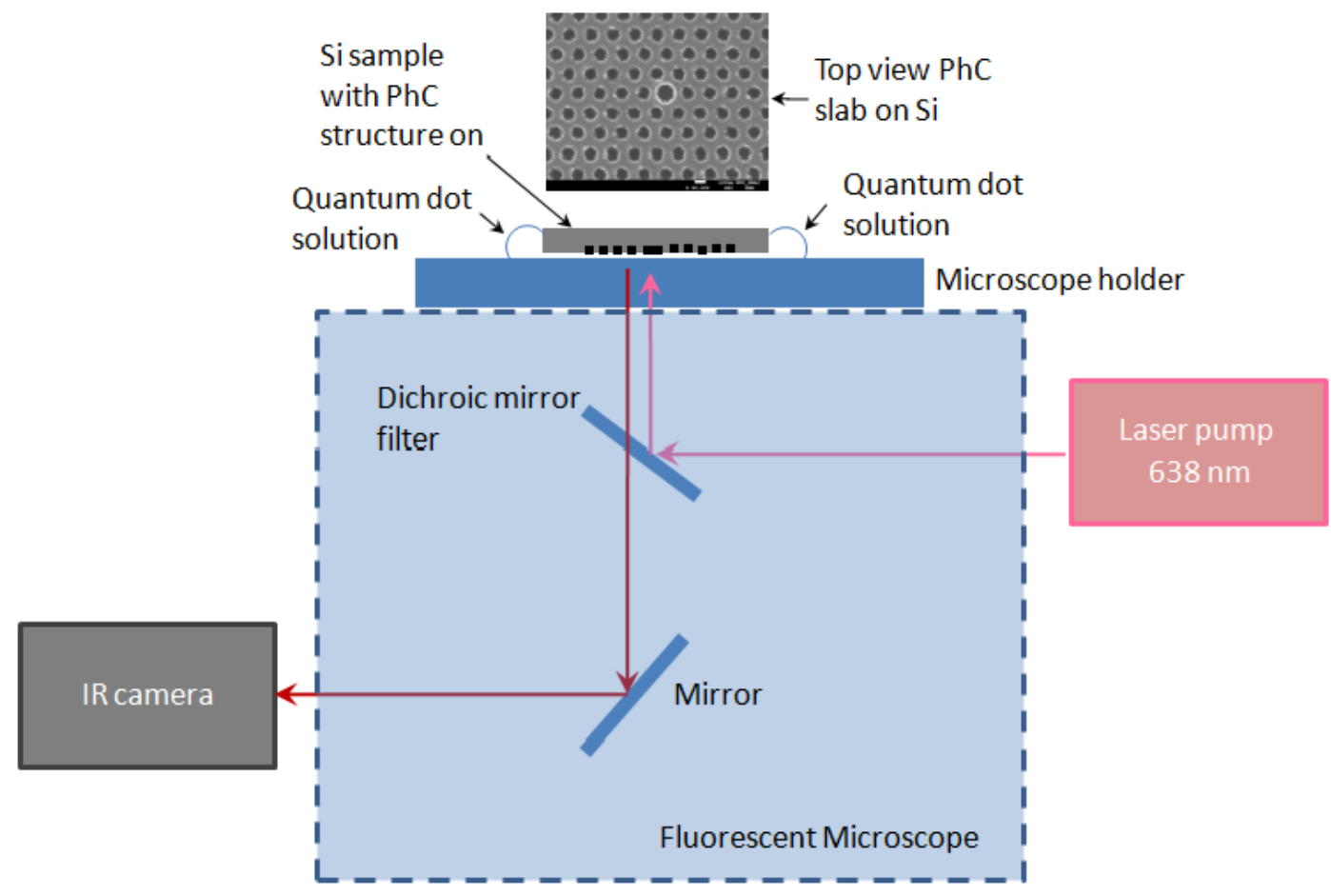

Figure 5-3: Schematic diagram of experiment for characterizing light enhancement inside the PhC nanocavity, system setup proposed and developed by Bashar Hamza.

This experiment setup is the initial effort for optical characterization of light confinement within the defect of the $\mathrm{PhC}$ slab using infrared quantum dots. The detailed characterization work is currently under way. Up to this point, no enhancement has been perceived, yet. This result has been attributed to the breakage of the $\mathrm{PhC}$ slabs when pressing the samples down on top of the quantum dot solution, against the microscope holder. To avoid structure breakage issues, new efforts will be conducted to improve the way of delivering small volumes of quantum dot solution into the $\mathrm{PhC}$ nanocavity using microfluific channels. Also to make the $\mathrm{PhC}$ structure more flexible and integrate it with the microfluidic channels, new PhC structures recipes will be elaborated to allow the fabrication of such structures in PDMS. 


\section{CONCLUSIONS AND FUTURE WORK}

In this thesis we developed the nanofabrication process for a new biosensor structure integrating a $\mathrm{PhC}$ nanocavity resonator with microfluidic channels. The EBL process was optimized in order to create high-resolution nanoscale $\mathrm{PhC}$ nanocavity patterns in both positive and negative tone e-beam resists. Two fabrication processes: (1) lift-off and (2) etch-back were developed to form the hard etch mask for subsequent $\mathrm{PhC}$ pattern transfer. The lift-off technique has proven to be capable of producing uniform metal mask with the sub-100 nm features. However, Etch-back as a second pattern transfer alternative using a positive e-beam resist resulted in $\mathrm{PhC}$ structures with a relatively poor resolution due to limited etching resistances of the e-beam resist and $\mathrm{SiO}_{2}$ during the pattern transfer by ICP etching. The plasma etching of $\mathrm{Si}, \mathrm{GaN}$, and ITO were optimized to obtain fast etching rates, vertical profiles as well as high etch selectivities over different hard masks including $\mathrm{Si}_{3} \mathrm{~N}_{4}, \mathrm{SiO}_{2}$, and $\mathrm{Ni}$. $\mathrm{BCl}_{3} / \mathrm{Cl}_{2}$ plasma yielded sidewalls of $\mathrm{GaN} \mathrm{PhC}$ airholes as steep as $87.6^{\circ}$ and etching rates of $85 \mathrm{~nm} / \mathrm{min}$. $\mathrm{BCl}_{3} / \mathrm{Cl}_{2} / \mathrm{CF}_{4}$ inductively coupled plasmas were studied for ITO etching. ITO etching rates over $200 \mathrm{~nm} / \mathrm{min}$ and sidewall profile angles of $88^{\circ}$ were obtained. These developed processes were used to demonstrate the successful fabrication of 2D PhC nanocavity structures in $\mathrm{Si}, \mathrm{GaN}$, and ITO with error ranging within 5\%.

To enable the integration of the $\mathrm{PhC}$ nanocavity resonator with a microfluidic channel, a glancing angle deposition recipe to grow a uniform $\mathrm{SiO}_{2}$ capping layer atop the $\mathrm{PhC}$ structure has been developed. It has been found that a combination of stationary and rotating deposition modes along with an exponential variation of the flux angle produced a thin uniform film $(\sim 300 \mathrm{~nm})$ which completely sealed the $\mathrm{PhC}$ airholes.

Finally, we fabricated suspended $\mathrm{PhC}$ nanocavity slab nanostructures on a SOI substrate. The structures provide important test beds for the characterization of light confinement and enhancement in the nanocavity resonators.

Future work includes the implementation of proximity effect correction tools to improve the resolution of $\mathrm{PhC}$ pattern writing and create sub $100 \mathrm{~nm}$ features in negative resists. Also plasma temperature in the ICP RIE chamber should be monitored in order to develop a solution to avoid resist burning during dry etching processes. With the etching issues, the etch-back technique could be further optimized to fabricate $\mathrm{PhC}$ structures with even smaller features. As for GLAD, implementation of an automation control will make possible continuous variations of the flux angle and the substrate rotation, which will likely produce much smoother films for capping the $\mathrm{PhC}$ structures, also these films need to be 
characterized to obtain the optical properties such as scattering effects of the grown film. Finally, suspended $\mathrm{GaN}$ PhC nanocavity structures will be fabricated in order to extend the fluorescence measurement into the visible and even UV wavelength regimes. This would require a specially-designed epitaxy structure, for instance, GaN/AlN grown-on-Si (111) substrates. 


\section{REFERENCES}

1. Prather, Dennis W, et al. PHOTONIC CRYSTALS Theory, Applications, and Fabrication. New Jersey : Wiley, 2009. 9780470278031.

2. Photonic crystals: a unique partnership between light and matter. Lipson, $\mathbf{R} \mathbf{H}$ and Lu, C. Canda : European Jornal of Physics, 2009, Vol. 30.

3. Photonic crystal: making a cage for light. Vos, Willem and Kuipers, L.

4. The Almost-Magical World of Photonic Crystal. Joannopoulos, J D. 1, USA : Brazilian Journal of Physics, 1995, Vol. 26.

5. Optical microcavities. Vahala, Kerry J. California : Nature, 2003, Vol. 424.

6. Photonic Band Structure: The Face-Centered-Cubic Case. Yablonovitch, E and Gmitter, T J. 18, New Jersey : Phisical Review Letters, 1989, Vol. 63.

7. High quality-factor whispering-gallery mode in the photonic crystal hexagonal disk cavity. Ryu, HanYoul, et al. 8, Japan : Optical Society of America, 2004, Vol. 12.

8. High-Q photonic nanocavity in a two-dimensional photonic crystal. Akahane, Yoshihiro, et al. Japan : Nature, 2003, Vol. 425.

9. High quality two-diensional photonic crystal slab cavities. Chen, Hao and Deppe, Dennis. 26, Texas : Applied Physics letters, 2001, Vol. 79.

10. Fabrication of high-quality-factor photonics crystak microcavities in InAsP/InGaAsP membranes.

Srinivasan, Kartik, et al. 3, New Jersey : American Vaccum Society, 2004, Vol. 22.

11. Hall, Elizabeth A. Biosensors. New Jersey : Prentice Hall, 1991. 0130845264.

12. Single-step fabrication and characterization of photonic crystal biosensors with polymer microfluidic channels . Choi, Charles J and Cunnigham, Brian T. Illinois : The Royal Society of Chemestry, 2006, Vol. 6.

13. Photonic crystal resonator integrated in a microfluidic system. Nunes, P S, et al. 14, Denmark: Optical Society of America, 2008, Vol. 33.

14. Two-dimensional silicon photonic crystal based biosensing platform for protein detection . Lee, Mindy and Fauchet, Philippe M. 8, New York : Optical Society of America, 2007, Vol. 15.

15. A label-free photonic crystal biosensor imaging method for detection of cancer cell cytotoxicity and proliferation . Chan, Leo L, et al. Illinois : Springer, 2007, Vol. 12. 
16. A Glucose Biosensor Based on Surface-Enhanced Raman Scattering: Improved Partition Layer, Temporal Stability, Reversibility, and Resistance to Serum Protein Interference. Yonzon, Chanda Ranjit, et al. 1, Illinois : American Chemical Society, 2003, Vol. 76.

17. Sensitive optical biosensor for unlabeled targets: A review. Fan, Xudong, et al. Missouri : Elsevier, 2008, Vol. 620.

18. Specific detection of proteins using photonic crystal waveguides. Buswell, S C, et al. 20, Canada : Optic Express, 2008, Vol. 16.

19. Photnic crystal fiber long period gratings for biochemical sensing. Rindorf, Lars, et al. 18, Denmark : Optical Society of America, 2006, Vol. 14.

20. Nanoscale microcavity sensor for single particle detection. Fauchet, Philippe M and Lee, Mindy R. 22, New York : Optical Society of America, 2007, Vol. 32.

21. Liquid-infiltrated photonic crystals: enhanced light matter interactions for lab-on-a-chip applications. Mortensen, Niels Asger, Xian, Sanshui and Pedersen, Jesper. Denmark : Springer, 2007, Vol. 4.

22. Developing optofluidic technology through the fusion of microfluidics and optics. Psaltis, Demetri, Quake, Stephen R and Yang, Changhuei. 27, California : Nature, 2006, Vol. 442.

23. Photonic crystal lasser sources for chemical detection. Loncar, Marko, Scherer, Axel and Qiu, Yueming. 26, California : American Institute of Physics, 2003, Vol. 82.

24. A Biomolecular Detection Device Employing an Optofluidic Photonic Crystal for Resonant Fluorescence Emission Enhancement. Dawson, J M, et al. Morgantown : s.n., 2007.

25. New Approaches to Nanofabrication: Molding, Printing, and Other Techniques. Gates, Byron D, et al. 4, Texas : American Chemical Society, 2004, Vol. 105.

26. Enabling Technologies for MEMS and Nanodevices. Haltes, H, et al. s.1. : WILEY-VCH, 2004, Vol. 1. 352730746X.

27. Technologies for nanofluidic systems: top-down vs. bottom-up - a review. Mijatovic, D, Eijkel, J C and Van den Berg, A. s.l. : The royal society of Chemistry, 2005, Vol. 5.

28. Gold Nanoparticles: Assembly, Supramolecular Chemistry,Quantum-Size-Related Properties, and Applications toward Biology, Catalysis, and Nanotechnology. Daniel, Marie-Christine and Astruc, Didier. France : American Chemical Society, 2004, Vol. 104.

29. Fabrication of novel biomaterials through molecular self-assembly. Zhang, Shuguang. 10, Massachusetts : Nature Biotechnology, 2003, Vol. 21.

30. Nanotechonology for Electronic Materials and Devices. korkin, Anatoly, et al. Canada : Springer, 2006. 9780387233499. 
31. Fabrication of Photonic Crystals in Silicon-on-Insulator Using 248-nm Deep UV Lithography. Bogaerts, Wim, et al. 4, Belgium : IEEE, 2002, Vol. 8.

32. JEOL. Instructions JEOL JSM-7600F. Japan : s.n., 2008.

33. Mc Cord, Mark A and Rooks, Michael J. SPIE Handbook of Microlithography, Micromachining and Microfabrication .

34. Brewer, George R. Electron-Beam Technology in Microelectronic Fabrication. California: Academic press, 1980. 0-12-133550-X.

35. Electron-beam-induced deposition using a subnanometer-sized probe of high-energy electrons.

Mitsuishi, K, et al. 10, Japan : American Institute of Physics, 2003, Vol. 83.

36. Aberration corretion in a low voltage SEM by multipole corrector. Zach, Joachim and Haider, Maximilian. Germany : Elsevier, 1995, Vol. 363.

37. Murata, Kenji, Kyser, David and Ting, Chiu H. Monter Carlo simulation of fast secondary electron production in electron beam resists. California : American Institute of Physics, 1980.

38. Determination of proximity effect parameters and the shape bias paremeter in electron beam lithography. Seo, Eunsung, Choi, Bo Kyung and Kim, Ohyun. Korea : Elsevier, 2000, Vol. 53.

39. Validity of Double and Triple Gaussian Functions for Proximity Effect Correction in X-ray Mask Writing. Aya, Sunao, et al. 3, Japan : Applied Physics, 1995, Vol. 25.

40. Methods for proximity effect correction in electron lithography. Owen, Geraint. California : Journal of Vacuum Science and Technology, 1990, Vol. 8.

41. Proximity effect corrstion in electron-beam lithography: A hierarchical rule-based scheme-PYRAMID. Lee, Soo Young, et al. 6, New York : Journal of Vacuum Science and Technology, 1991, Vol. 9.

42. Proximity effect correction by the GHOST method using a scattering stencil mask. Yanashita, Hiroshi, et al. 6, Japan : Journal of Vacuum Science and Technology, 1999, Vol. 17.

43. Campbell, Stephen A. The Science of Engineering of Microelectronic Fabrication. New York: Oxford University Press, 1996. 0195105087.

44. Doyle, John M. Thin-Film and Semiconductor Integrated Circuitry. New York: Mc Graw-Hill, 1996.

45. Liu, Ai-Qun. Photonic MEMS Devices. New York : CRC press, 2009. 9781420045680.

46. Elliot, David. MICROLITHOGRAPHY PROCESS TECHNOLOGY FOR IC FABRICATION. New York : Mc Graw-Hill, 1986. 0070193045.

47. Effects of plasma conditions on the shapes of features etched in CL2 and HBr plasmas. I. Bulk crystalline silicon etching. Vyvoda, M A, et al. 6, New Jersey: American Vacuum Society, 1998, Vol. 16. 
48. Nanoscale pattern transfer for Templates, NEMs, and Nano-optics. Olynick, Deirdre L, et al. California : Proc. of SPIE, 2007, Vol. 6462.

49. Nightingale, Joshua Ryan. OPTICAL BIOSENSOR: SPARROW BIOSENSOR AND PHOTONIC CRYSTAL-BASED FLUORESCENCE ENHANCEMENT. West Virginia : s.n., 2008.

50. Yeldandi, Satish. Development of Fabrication Processes for Si and GaN Photonic Crystal Structures. West Virginia : s.n., 2008.

51. Fabrication of microelectrode arrays for neural measurements from restinal tissue. Cunningham, W, et al. California : Applied Physics, 2001, Vol. 34.

52. Electrophoretic assembly of colloidal crystals with optically tunable micropatterns. hayward, $\mathbf{R} \mathbf{C}$, Saville, D A and Aksay, I A. 2, New Jersey : Nature, 2000, Vol. 404.

53. Indium Tin Oxide-Polyaniline Biosensor: Fabrication and Characterization. Tahir, Zarini Muhammad, Alocija, Evangelyn C and Grooms, Daniel L. Michigan : Sensors, 2007, Vol. 7.

54. Oblique-Angle Sputtering Effects on Characteristics of Nanocolumnar Structure Anisotropic Indium Tin Oxide Films. Lung-Chien Chen, Cheng-Chiang Chen, Yeng-Tang Sung, Ya-Ying Hsu. 6, Taipei : Electrochemical Society, 2009, Vol. 1556.

55. Aligned amorphous and microcrystalline Si nanorods by glancing angle depostition at low temperature. Yanhong, Ma, et al. Beijing : IOP publishing, 2009, Vol. 20.

56. Glancing angle depostition to modify microstrusture and properties of sputter deposited chromium thin films. Lintymer, J, et al. France : Surface and Coating Technology, 2003.

57. Oblique Angle Deposition of Germanium Film on Silicon Substrate. Chew, H G, et al. Singapore: s.n.

58. Tailoring growth and local composition by oblique-incidence deposition: a review and new experimental data. Kranenburg, Herma Van and Lodder, Cock. Netherlands : Materials Science and Engineering, 1994.

59. Advanced techniques for glancing angle depostition. Robbie, K, Sit, J c and Brett, M J. alberta : American Vaccum Societe, 1997, Vol. 16.

60. Empirical equations for the principal refractive indices and column angle of oblique deposited films of tantalum oxide, titanium oxide, and zirconium oxide. Hodgkinson, Ian, Wu, Qi Hong and Hazel, Judith. 13, New Zealand : Applied Optics, 1997, Vol. 37.

61. Modelling and characterization of columnar growth in evaporated films. Tait, R N, Smy, T and Brett, M J. 2, 1993, Vol. 226.

62. The growth of nanoscale structured iron films by glancing angle deposition. Liu, F, et al. 8, Alabama : Journal of Applied Physics, 1999, Vol. 85. 
63. Ultrahigh vacuum glancing angle deposition system for thin films with conttrolled three dimentional nanoscale structure. Robbie, Kevin, et al. 4, Ontario : American Institute of Physics, 2004, Vol. 75. 


\section{APPENDIX A}

\section{CAD File design for 2D hexagonal PhCs}

Previously, it is essential to create a project in NPGS software and open DesignCAD Express 16 from the NPGS interface. This will create all the auxiliary files in your project folder in order to generate the respective run files later. Once Design CAD Express 16 is open, draw a hexagon with side equals to the lattice constant a and then measure L. For PhC patterns, polygons will be always used, therefore, dashed line should be the types of line to be used in any desing. Draw a circle with a radius equals $r$ right on one corner of the hexagon, use right click to place the circle exactly on the corner.

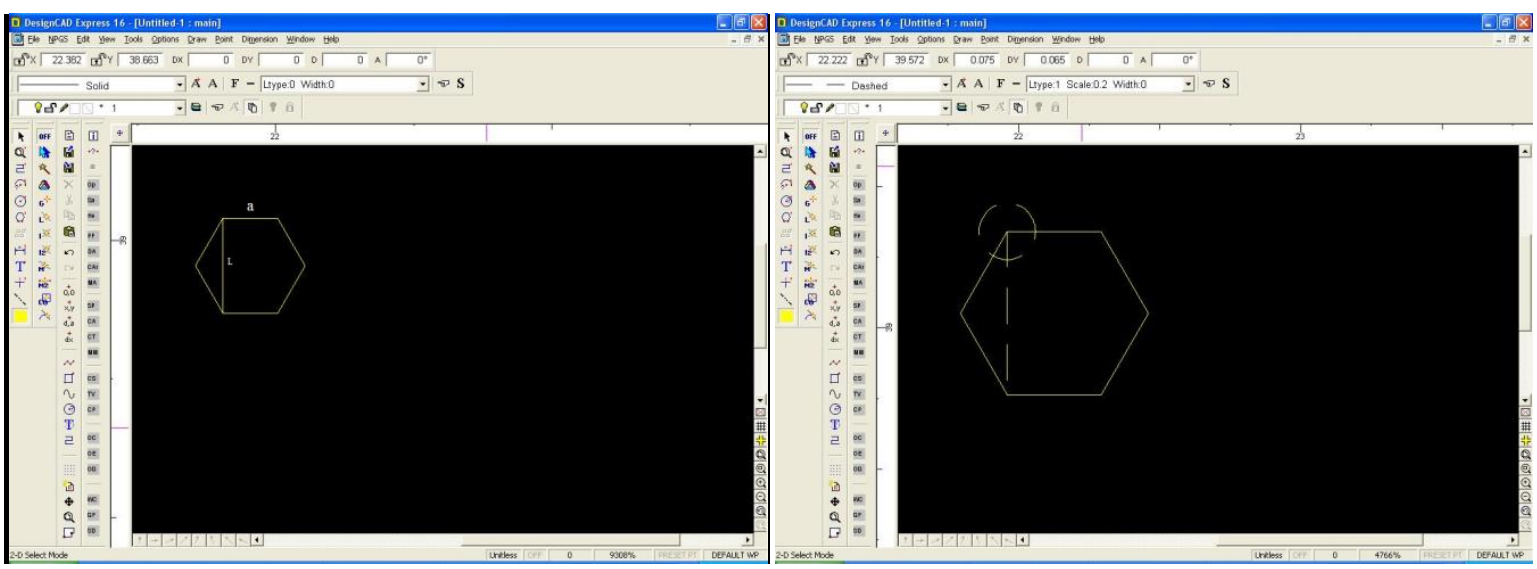

Then, use the command MA (make array) from the NPGS menu to create a row as long as needed of circles. When using MA command, the distance between columns should be the lattice constant. Draw a second circle as shown in the plot below.

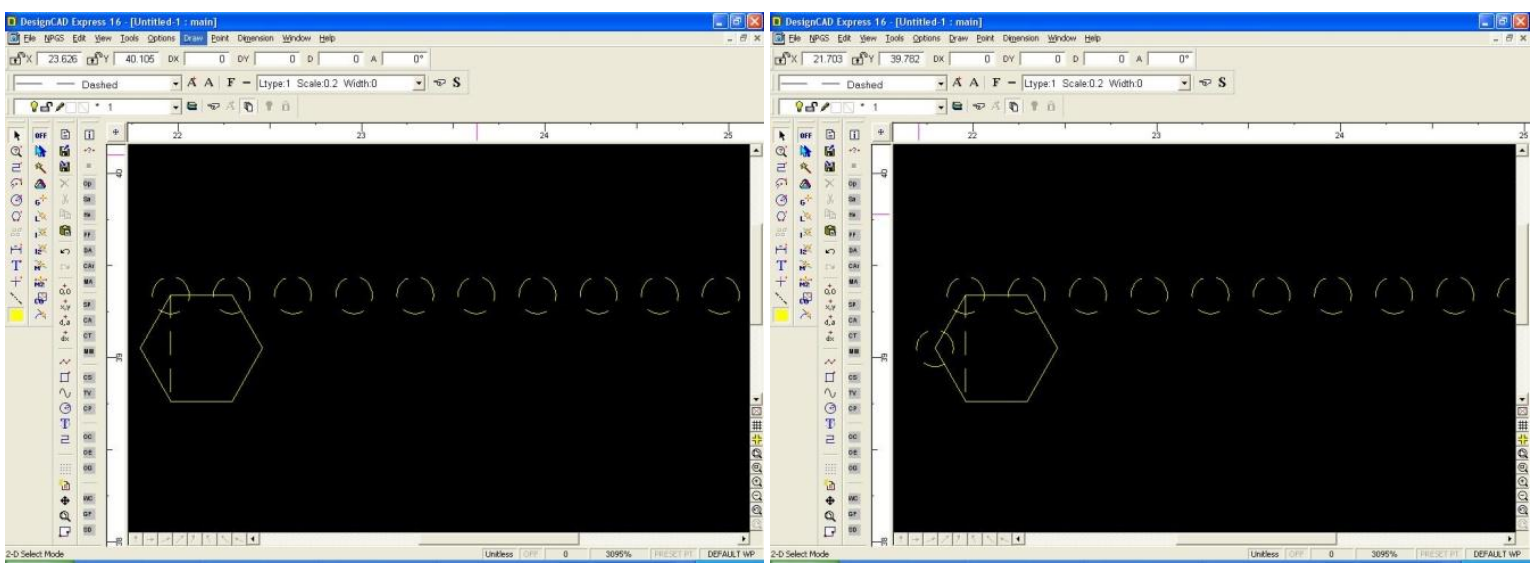

Next use again the command MA to create a row similar to the previous one with column spacing equals to the lattice constant a and the same number of columns. Now the hexagon should be deleted and any other auxiliary line. 


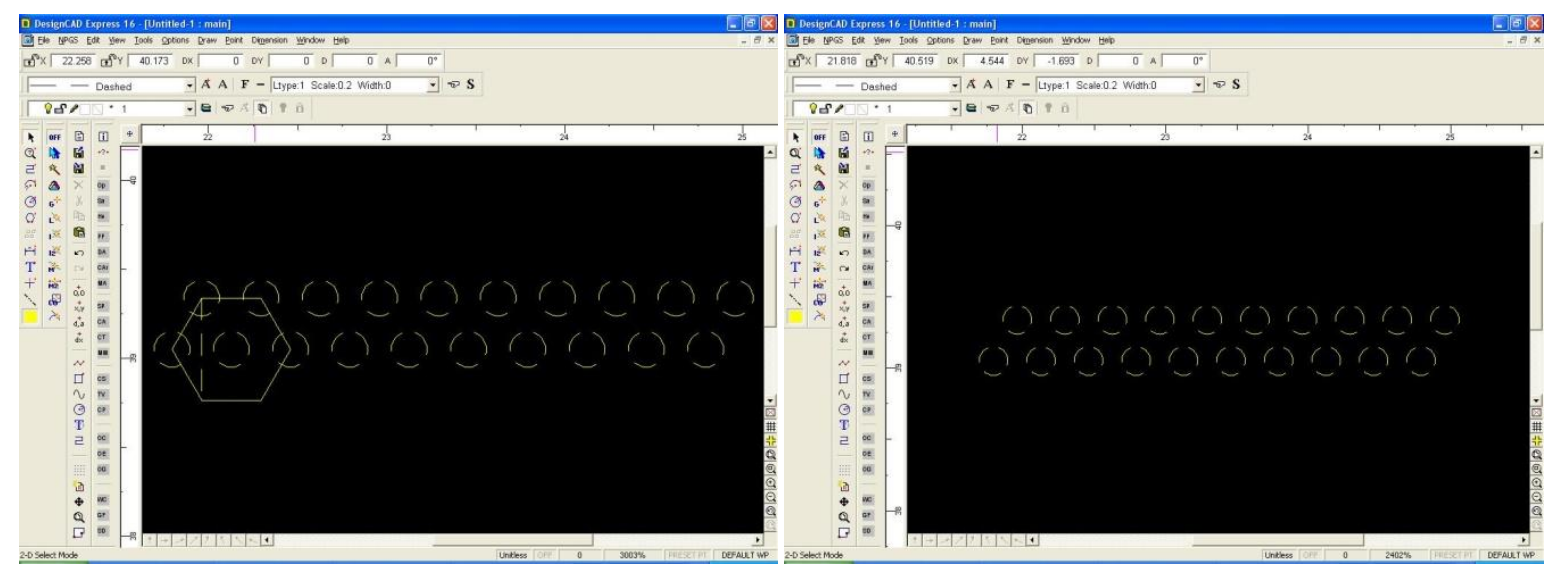

MA command should be used again to create the final $\mathrm{PhC}$ pattern. Select the rows that have created to make an array of one column and spacing between rows equals parameter L, which was measured at the beginning. The number of rows should be according to how big your $\mathrm{PhC}$ structure is. It is highly recommended to run the command CA (check all) to double check if the files has some errors. The plots below show a final $\mathrm{PhC}$ pattern and the command window $\mathrm{CA}$.

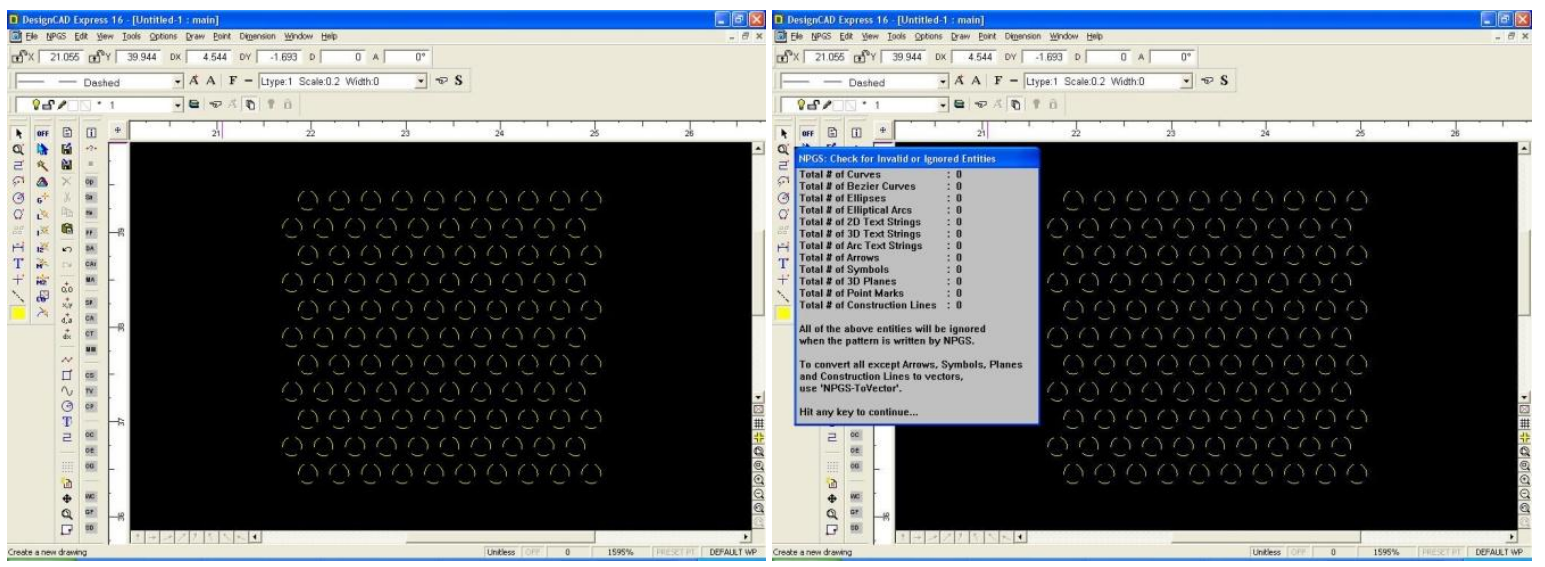

The following are some shortcuts that are very helpful.

' shows a windows for determining dimension when drawing some feature.

Crtl W zooms your pattern such it fit the screen.

Crtl I shows the properties of any feature.

$\mathrm{H}$ creates orthogonal lines

$\mathrm{V}$ creates lines with any direction

Crtl $\mathrm{H}$ sets handles at any position. It can help when long patterns are needed to be handled. This command can change the origin of any selection to any position.

Please refer to the manual for detailed information on CAD file design. 


\section{APPENDIX B}

\section{RUN FILE in NPGS}

Once a CAD file has been created, from NPGS right click on it and select run file editor. This will generate a file that will communicate with the SEM in order to conduct the writing of the designed pattern. The figure below shows a run file for the pattern shown in Fig. 3.1. This file contains the parameters of writing, which were used in this work for negative resist.

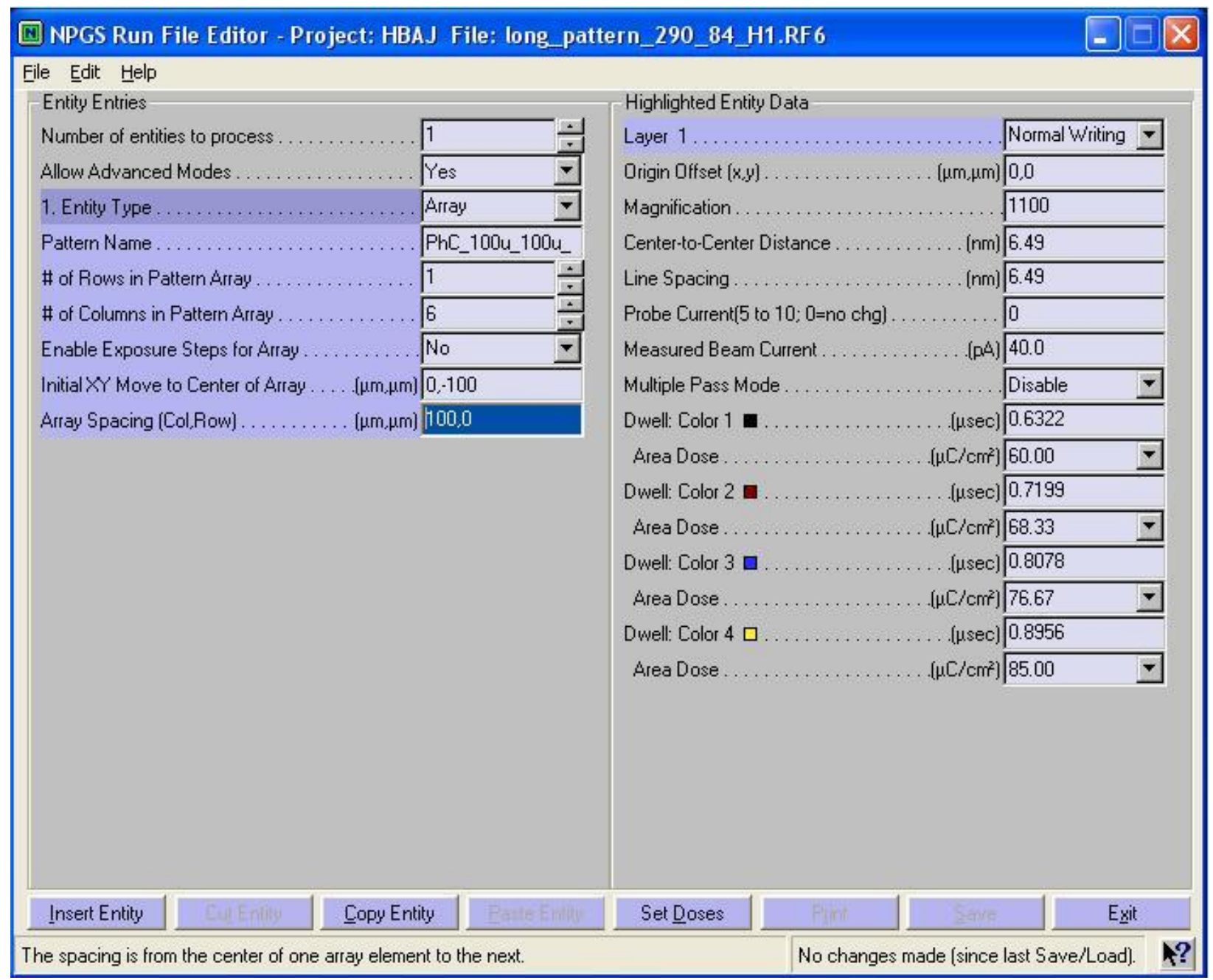

Please refer to the NPGS manual for detailed information on RUN file parameters.

John $\mathrm{H}$.

Digitally signed by John $\mathrm{H}$. Hagen 